1

2

3

\title{
The human in vivo biomolecule corona onto PEGylated liposomes: a proof-of-concept clinical study
}

\author{
Marilena Hadjidemetriou1, Sarah McAdam², Grace Garner², Chelsey Thackeray ${ }^{3}$, David Knight, Duncan
} Smith $^{5}$, Zahraa Al-Ahmady ${ }^{1}$, Mariarosa Mazza ${ }^{1}$, Jane Rogan², Andrew Clamp³ and Kostas Kostarelos ${ }^{1 *}$

${ }^{1}$ Nanomedicine Lab, Faculty of Biology, Medicine \& Health, AV Hill Building, The University of Manchester, Manchester, United Kingdom; ${ }^{2}$ Manchester Cancer Research Centre Biobank, The Christie NHS Foundation Trust, CRUK Manchester Institute, Manchester, United Kingdom ${ }^{3}$ Institute of Cancer Sciences and The Christie NHS Foundation Trust, Manchester Cancer Research Centre (MCRC),

University of Manchester, Manchester, United Kingdom

${ }^{4}$ Bio-MS Facility, Michael Smith Building, The University of Manchester, Manchester, United Kingdom;

${ }^{5 x}$ CRUK Manchester Institute, The University of Manchester, Manchester, United Kingdom

\footnotetext{
$\overline{{ }^{*} \text { Correspondence should be addressed to: kostas.kostarelos@manchester.ac.uk }}$
} 


\begin{abstract}
The self-assembled layered adsorption of proteins onto nanoparticle (NP) surfaces, once in contact with biological fluids, has been termed the 'protein corona' and it is gradually seen as a determinant factor for the overall biological behavior of NPs. Here, we describe the previously unreported in vivo protein corona formed in human systemic circulation. The human-derived protein corona formed onto PEGylated doxorubicin-encapsulated liposomes (Caelyx®) was thoroughly characterized following the recovery of liposomes from the blood circulation of ovarian carcinoma patients. In agreement with our previous investigations in mice, the in vivo corona was found to be molecularly richer in comparison to its counterpart ex vivo corona. The intravenously infused liposomes were able to scavenge the blood pool and surface-capture low molecular weight, low abundant plasma proteins that could not be detected by conventional plasma proteomic analysis. This study describes the previously elusive or postulated formation of protein corona around nanoparticles in vivo in humans and illustrates that it can potentially be used as a novel tool to analyze the blood circulation proteome.
\end{abstract}

Keywords: biomolecule corona, Doxil@, ovarian cancer, nanomedicine 


\section{Introduction}

The surface identity of nanoparticles (NPs) is modified once injected in the bloodstream because of their spontaneous interaction with a wide range of surrounding proteins and other biomolecules. ${ }^{[1]}$ The spontaneous and layered adsorption of proteins onto NPs has been termed the 'protein corona'. ${ }^{[2]}$ Even though surface modification of NPs with hydrophilic polymers (e.g. PEGylation) results in diminution of protein adsorption onto NPs surfaces, the complete elimination of protein corona formation has not been achieved so far by such functionalization strategies. ${ }^{[3-7]}$ During the last decade protein corona research has gained popularity with a flurry of attempts made to molecularly characterize corona profiles after the ex vivo incubation of NPs with biofluids (mainly plasma) and more recently, in vivo (in rodents). ${ }^{[4-7]}$

The biological effects arising from the presence of biomolecules onto NPs surfaces can be both unfavorable and advantageous to their biomedical applications. ${ }^{[1,8]}$ It is now reasonably established that the protein corona defines the NP surface characteristics and forms the interface between NPs and biological systems, also referred by some to as the 'bio-nano' interface. The presence of the protein corona mediates the interaction of NPs with cells and has been shown to significantly impact on their cytotoxicity, cellular internalization and targeting capability. ${ }^{[9,10]}$

Among a plethora of nanoscale drug delivery systems, liposomes (phospholipid-based vesicles) are considered to be the most clinically-established nanomedicine constructs. ${ }^{[11]}$ The substantial reduction in the cardiotoxicity of doxorubicin upon encapsulation within PEGylated liposomes led to the approval of the first nanoscale anti-cancer agent (Doxil囚, Caelyx®) by the FDA in 1995 for the treatment of for AIDS-related Kaposi Sarcoma and in 1999 for the treatment of recurrent ovarian carcinoma. ${ }^{[12,13]}$ This liposomal construct shows superiority to free doxorubicin owing to its prolonged blood circulation time, the stable retention of the drug in the interior of liposomes while in blood circulation, leading to an overall improved toxicity profile and enhanced tumor accumulation. ${ }^{[12,14]}$

Despite the clinical track record of liposomes for more than 20 years, the role that protein corona plays in liposomal pharmacology has been barely studied. To date, only a few studies describe the molecular composition of the protein corona formed around NPs in vivo. ${ }^{[4-6,15]}$ In the vast majority of investigations, NPs are characterized after their ex vivo interaction with plasma proteins (mouse or human), extrapolating on the erroneous assumption that corona fingerprints formed under such a static biological setting can reflect the dynamics and molecular richness of the in vivo milieu. ${ }^{[1]}$

In our previous studies, we developed a robust protocol to retrieve and purify NPs from blood 
to investigate the in vivo protein corona formation and its evolution onto clinically-used liposomes after their intravenous administration (tail vein) in rodents. These studies revealed that the molecular composition of the in vivo protein corona cannot be sufficiently predicted by the ex vivo plasma incubation of NPs. ${ }^{[4-6]}$ Blood flow dynamics, the interaction with blood circulating cells and immune responses triggered after NPs administration are some of the factors that cannot sufficiently be simulated by ex vivo studies and could explain the differences observed between the ex vivo and in vivo formed protein coronas. ${ }^{[1]}$ Although in vivo models shed light on our understanding of the selfassembly mechanism of the protein corona formation under more biologically-relevant settings, extrapolation of data from mice to humans should be made with extreme caution. ${ }^{[16]}$ Nanoparticlebased systems that work efficiently in rodent disease models often fail to offer similar efficacy in humans. ${ }^{[17]}$ Yet, the formation of a protein corona around blood-circulating nanoparticles in humans has not been experimentally described at all, let alone studied as a potential factor to explain such discrepancies between preclinical and clinical achievements.

In the present study, we attempted to investigate and characterize the in vivo protein corona formation in humans. PEGylated, doxorubicin-encapsulated liposomes (Caelyx®) were infused intravenously in six patients with platinum-resistant recurrent ovarian carcinoma and subsequently recovered from the blood circulation immediately on completion of their first cycle of Caelyx® treatment. Liposomes were isolated from blood components along with their formed protein corona which was quantitatively and qualitatively characterized using a battery of techniques. The data indicated that an in vivo human protein corona forming around intravenously-infused liposomal nanoparticles can be reproducibly identified and molecularly described. The human in vivo protein corona was rich in multiple low molecular weight and low abundant plasma proteins that could not be detected by conventional plasma proteomic analysis, which revealed the potential utilization of the biomolecule corona as a tool to address the issue of the high dynamic range of plasma proteome. 


\section{Results and Discussion}

\section{Physicochemical characterization of liposomes and human in vivo corona-coated}

liposomes. To characterize the in vivo formed, human-derived protein corona, PEGylated doxorubicin-encapsulated liposomes were recovered from the blood circulation of ovarian carcinoma patients $(n=6)$ at the end of their first cycle of intravenous Caely $x^{\circledR}$ infusion (at a dose of $40 \mathrm{mg} / \mathrm{m}^{2}$ ), for platinum-resistant disease (Figure 1A). Patient clinical and basic blood analysis characteristics are summarized in Table S1. The physicochemical properties of the commercially available PEGylated doxorubicin-encapsulated liposomes (Caelyx ${ }^{\circledR}$ ) were investigated by dynamic light scattering (DLS), $\zeta$-potential measurements and negative stain transmission electron microscopy (TEM) before and after their intravenous infusion in patients and are summarized in Figures 1B and 1C. Liposomes had a mean hydrodynamic diameter of $82.6 \mathrm{~nm}$, a negative surface charge of $-33.8 \mathrm{mV}$ and displayed low polydispersity values $(<0.1)$ representing a narrow size distribution (Figure 1B). TEM imaging showed well-dispersed, drug-encapsulated round shaped vesicles, with their size correlating that of DLS measurements (Figure 1C).

Immediately after the completion of the intravenous infusion of Caelyx ${ }^{\circledR}$ (that lasted for approximately 90 mins), blood ( 10ml) was collected and plasma was prepared without delay by centrifugation. A two-step purification protocol (size exclusion chromatography and membrane ultrafiltration) was then employed for the isolation of liposome-corona complexes and the complete elimination of unbound proteins, as we have previously described (Figure S1). ${ }^{4,5]}$ It should be noted that although the protein corona is often described as a multi-layered structure consisting of an inner layer of tightly bound proteins ('hard corona') and an outer dynamically-bound layer of proteins ('soft corona'), current purification protocols might disturb loosely bound biomolecules. Therefore, the existence and the biological relevance of the 'soft corona' remain unclear and we can only refer to the analytically accessible protein corona. ${ }^{[18]}$

Dynamic light scattering measurements of corona-coated liposomes demonstrated that their size distribution broadened (Figure 1B). Formation of the corona is often associated with an increase in the mean nanoparticle diameter since layers of proteins are adhered onto the NP surface. In the case of soft nanomaterials however, adsorption of proteins could also lead to a reduction in their mean diameter due to osmotic "shrinkage". This has been previously reported ${ }^{[4,5}$, ${ }^{19]}$ and also observed here in the case of human in vivo and ex vivo corona-coated liposomes (Figures 1B and S2). In addition, our data showed that the mean surface charge of liposomes remained negative after their interaction with plasma proteins (Figure 1B and S2) in agreement with previous studies proposing that negatively charged NPs do not exclusively interact with 
positively charged proteins, as electrostatic interactions are not the only driving force at the NPprotein interface. ${ }^{[20]}$

Moreover, TEM imaging revealed well dispersed and corona-coated liposomes that retained an intact structure after recovery and purification (Figure 1C). Although protein corona is usually illustrated as a dense layer covering the entire surface of a nanoparticle, this has not been experimentally confirmed. In agreement with our previously reported observations in rodents, ${ }^{[4,5]}$ the human in vivo protein corona did not appear to coat entirely the liposome surface. Cryo-EM imaging of the recovered liposomes further confirmed the presence of a protein corona adsorbing around the doxorubicin-encapsulated $\left(\right.$ Caely $x^{\circledR}$ ) liposomes, but without fully covering their surface (Figure 1C). Similarly, Kokkinopoulou M et al., have recently described the protein corona formed around polystyrene NPs as an undefined and unfolded network surrounding the NP surface. ${ }^{[21]}$ Moreover, small vesicular structures surrounding the liposome surface could be observed by TEM in Figure 1C. We hypothesize that these could be either osmotically shrunk liposomes or bloodcirculating extracellular vesicles adsorbed onto the surface of liposomes. Based on these observations, further studies will be needed to explore the adherence of other molecules or vesicular structures onto the surface of blood-circulating NPs.

Characterization of the human in vivo protein corona. Previous anti-opsonisation studies have emphasized that the total amount of protein adhered onto NPs can be used to predict the NPs blood circulation time. ${ }^{[22-24]}$ Even though the overall protein adsorption is moderated by the PEG chains present onto the NPs surface, it cannot be fully suppressed. ${ }^{[3-5]}$ In agreement with these previous findings, the present study demonstrated that PEGylated nanoscale surfaces are not entirely inert and interact with plasma proteins upon intravenous administration in humans.

To investigate the total amount of protein adsorbed, we calculated the protein binding ability $(\mathrm{Pb})$, defined as the amount of protein associated with each umole of lipid. As shown in Figure 2A, the average $\mathrm{Pb}$ value observed was $1532 \mu \mathrm{g}$ of protein/ $\mu$ mole of lipid, that is more than 10 times higher than what was obtained for the same liposome composition (produced in the laboratory) after injection in healthy CD-1 mice. ${ }^{[5]}$ Whether this is a result of mouse-to-human differences and/or due to the neoplastic disease present at an advanced stage in the patients that participated in this study, is difficult to conclude and will require further investigation. Ex vivo incubations of Caely $x^{\circledR}$ liposomes with plasma samples, obtained from the same patients before Caelyx ${ }^{\circledR}$ infusion were performed as a control. As shown in Figure S3, a significantly lower average $\mathrm{Pb}$ value was observed which confirms our previous data suggesting that the ex vivo incubation of NPs with 
human plasma samples cannot predict the formation of protein corona under realistic in vivo conditions. ${ }^{[4]}$

The manner in which proteins adsorb and pack onto the NP surface is highly dependent on their physicochemical properties and especially their size, shape and functionalization. However, concrete relationships between the nanomaterial synthetic identity and their ensuing biological identity in physiological environments remain vague and unpredictable. ${ }^{[1]}$ Distinct proteins could be either enriched or displayed weak affinity for the NP surface depending on the balance between their rates of association $\left(K_{o n}\right)$ and dissociation $\left(K_{o f f}\right){ }^{[25]}$ It has previously been shown by us in vivo ${ }^{[5]}$ and others in vitro ${ }^{[26,27]}$ that the protein corona is a temporally dynamic entity. In complex biofluids, such as blood, proteins present at high concentrations are characterized by high $K_{o n}$ values and therefore have high possibility to interact with the surface of NPs. However, these proteins might be replaced by other molecules of lower abundance, but of higher binding energy (characterized by low $K_{\text {off }}$ values). Our previous time evolution studies in rodents revealed that a molecularly rich in vivo protein corona was formed around PEGylated liposomal doxorubicin $\left(\right.$ Caelyx $\left.{ }^{\circledR}\right)$ as early as 10 min post-injection. Even though the total amount of protein adsorbed and the identity of the corona proteins did not significantly change, the abundance of each protein fluctuated over time, indicating that competitive exchange processes were taking place. Interestingly, liposomes were coated by a complex mixture of low MW proteins at all different time points of investigation. ${ }^{[5]}$

To examine whether our previous observation in rodents applies also under the in vivo conditions in humans, we comprehensively identified the protein molecules that self-assembled to form the corona around the intravenously infused liposomes by mass spectrometry. Surface-bound proteins were classified according to their molecular weight. As illustrated in Figure 2B, plasma proteins with $\mathrm{MW}<80$ accounted for almost $80 \%$ of the protein coronas formed. It is possible that the low MW proteins identified (Figure 2B) have high affinity and interact directly with the surface of PEGylated liposomes and/or they are trapped between other corona-carrier proteins that are adhered to the NPs surface.

To further understand the protein composition of the in vivo human corona, the average value ( $n=6$ patients) of relative protein abundance (RPA) for each identified protein was calculated. Figure 2C summarizes the 20 most abundant proteins associated onto the surface of recovered Caely ${ }^{\circledR}$ liposomes for all patients. The most abundant corona protein was full-length cDNA clone CS0DD006YL02 (with accession number Q86TT1; SwissProt database). To the best of our knowledge, this protein has not been previously reported to associate with the surface of liposomes or any other type of nanoparticle after their incubation in full plasma. It has been only previously 
shown to interact with maltose-functionalized PEGylated hybrid magnetic NPs after their incubation with fractionated human plasma sample. ${ }^{[28]}$ The 20 most abundant proteins identified in each patient and their respective RPA values are also shown in Table $\mathbf{S} 2$ to further illustrate the consistency of the above observation. The fact that the most abundant corona proteins were common between the 6 patients illustrates the consistency of liposome-protein interactions. Out of 445 corona proteins identified, 122 were repeatedly detected in all patients (Figure S4 and Table S5).

In agreement with our previous investigations of the liposomal protein coronas in mice, ${ }^{[5]}$ immunoglobulins, lipoproteins and complement proteins were the most abundant classes of proteins, contributing to $28 \%, 9 \%$ and $4 \%$ of the total protein content respectively (Figure $2 \mathrm{D}$ and Tables S2-S3). The presence of opsonins (such as immunoglobulins), known to activate the mononuclear phagocytic system (MPS), can favor recognition and clearance of the bloodcirculating PEGylated liposomes used in this study. However, the PEG-mediated inhibition of interactions between nanoparticles and circulating blood cells has been proposed to explain their long circulation time. ${ }^{[29]}$ As the molecular identification of the human corona suggests, lipoproteins were the second most abundant class of proteins, proposed to have dysopsonic activity (i.e. favor long blood circulation), possibly via competitive binding for the liposomal surface with opsonic proteins. ${ }^{[30]}$ The high affinity and adherence of lipoproteins to the lipid surface of Caelyx ${ }^{\circledR}$ NPs observed, was not surprising considering that blood circulating lipoproteins are involved in lipid and cholesterol transport and metabolism.

Intravenously infused, doxorubicin-encapsulated PEGylated liposomes have also been shown to interact with the complement system, in some cases triggering a transient and mostly mild hypersensitive reactions known as C-activation related pseudoallergy (CARPA). ${ }^{[31,32]}$ Despite the presence of several key complement cascade proteins in the liposomal corona involved in the classical (complement C1s and C1qb, C4b binding protein) alternative (complement factor $\mathrm{h}$, complement C3) and in lectin (mannan-binding lectin serine protease) pathways of activation (Figure 2D and Table S3), none of the patients in our study experienced a clinically symptomatic reaction to Caelyx® infusion.

Patient-by-patient blood-circulation proteome analysis. Blood predominantly contains high abundance, high MW proteins, such as albumin and immunoglobulins, that hamper the detection of the lower MW blood proteomic fractions. ${ }^{[33]}$ There is an urgent need for tools to facilitate the discovery of new and, more importantly, combinations of protein molecule panels to improve early cancer diagnosis, evaluate disease progression and monitor response to the treatment. Robust 
high-throughput proteomic discovery platforms that will enable the identification of blood-buried molecules are of immediate clinical importance.

The fact that albumin 'masking' was largely eliminated from in the in vivo human-derived corona prompted us to investigate further whether liposomes interacted with plasma proteins that could not be directly detected by plasma proteomic analysis. Proteins associated with liposomes in vivo were separated by SDS-PAGE and visualized by Imperial Protein stain, as illustrated in Figure 3A. Contrary to plasma control, the distinct bands of corona proteins, even at the low MW region, confirmed the ability of liposomes to surface-capture low abundant proteins minimizing the 'noise' from highly abundant proteins (Figure 3A).

Low molecular weight proteins are easily cleared from blood circulation (mainly through proteolytic plasma clearance), which limits their detection by conventional plasma proteomic analysis. ${ }^{[34-36]}$ The only way a small molecule can remain in the blood circulation for longer periods is to adhere to a long-circulating high abundance protein, such as albumin. ${ }^{[35,36]}$ The adherence of smaller MW biomolecules onto the surface of NPs once injected in the bloodstream, could significantly increase their blood circulation and allow their successful 'enrichment' for detection. Therefore, the characterization of the purified corona proteins can be employed as a 'fractionation' plasma tool that addresses the signal-to-noise challenge.

The nanoparticle-mediated capture of low MW proteins from biofluids has been so far attempted exclusively ex vivo. For instance, Nanotrap $\AA^{\circledR}$ technology developed by Liotta and coworkers, uses core shell hydrogel nanoparticles as protein harvesters. This technology is based on the NP-mediated size and charge dependent fractionation of complex biofluids, such as plasma and urine, prior to proteomic analysis. The porous outer shell of NIPAm (N-isopropylacrylamide) NPs blocks the entry of high MW proteins, while the internal core contains covalently attached chemical affinity baits which capture low MW proteins. ${ }^{[37,38]}$

To compare the human corona proteins with the plasma proteome, we analyzed plasma samples obtained from the same patients before the infusion of Caelyx ${ }^{\circledR}$. The Venn diagram in Figure 3B illustrates the number of common and unique proteins between the liposomal corona and plasma, as identified by mass spectrometry. A significantly higher total number of proteins was detected in the corona samples in comparison to the number of proteins identified when plasma samples were analyzed (Figure 3B). In addition, the most abundant plasma proteins were not the predominant corona proteins, as depicted in Figure $\mathbf{3 C}$ and Tables $\mathbf{S 3}$ and S4. Strikingly, the most abundant corona protein (full-length cDNA clone, CS0DD006YL02), which contributed to $8 \%$ of the total protein content, was not detected in any of the plasma control samples (Figure $\mathbf{3 C}$ and Table S4). Although, full-length cDNA clone CSODD006YL02 has been previously identified by mass 

proteomic approaches fail to detect this low abundant protein in complex mixtures like plasma.

The above findings prompted us to further investigate the molecular composition of the ex vivo protein corona after the incubation of Caelyx ${ }^{\circledR}$ liposomes with plasma samples obtained from the same ovarian carcinoma patients before Caely ${ }^{\circledR}$ infusion. In agreement with our previous data in rodents, a more complex molecular fingerprint was detected for the in vivo protein corona in comparison to its counterpart ex vivo corona (Figure S5). Despite the fact that the cDNA clone CS0DD006YL02 protein was not detected by mass spectrometry in any of the control plasma samples, it was identified as the most abundant protein of both the in vivo and ex vivo formed protein coronas (Figure 2A and Table S6). Control investigations of the ex vivo corona formed onto Caelyx® liposomes upon incubation with plasma samples from healthy volunteers (Table S6) were also performed. The cDNA clone CS0DD006YL02 protein was found to be the $5^{\text {th }}$ most abundant protein in the control cohort suggesting that tumorigenesis may affect the dynamics of corona formation. Overall, the above data provide an initial evidence that the liposome protein corona results in an 'enriched' sampling of the blood proteome which renders the need for much more work on the biomarker discovery front necessary, but beyond the scope of this study.

Previously unreported experimental evidence that a biomolecule corona self-assembles around nanoparticles in humans while in their blood circulation has been offered in the present study. The successful recovery and purification of corona-coated lipid bilayer vesicles from the blood circulation of ovarian carcinoma patients allowed the proteomic analysis of the human in vivo protein corona. We demonstrated that the clinically-used liposomal nanoparticles interact and can be stably coated with a complex mixture of plasma proteins, including low MW and low-abundant molecules otherwise 'masked' under the overwhelming signal of highly abundant proteins (such as albumin and immunoglobulins). The corona-based elimination of this 'masking' effect, enabled the uncovering of multiple protein molecules that could not be detected by plasma sample analysis performed in comparison. This work is thought to act as the impetus for many future studies needed to improve our further understanding of how the human in vivo biomolecule corona can affect the overall clinical performance of NPs, but also provide the technology springboard to allow the clinical exploitation of protein corona fingerprinting as a novel tool to comprehensively analyze the blood circulation proteome 


\section{Experimental}

Ethical Approvals. This project was reviewed and approved by the Manchester Cancer Research Centre Biobank Sample Access Committee and all sample collection was conducted under the MCRC Biobank Research Tissue Bank Ethics (ref: 07/H1003/161+5).

Blood sample collection. Caelyx $\AA$ is indicated for the treatment of advanced ovarian cancer in women who have failed a first-line platinum-based chemotherapy. Patients receive in total 6 cycles of Caelyx with cycle frequency 28 days. Eligible cases for this study included women with recurrent ovarian cancer commencing single agent Caelyx ${ }^{\circledR}$ for the first time, at a dose of $40 \mathrm{mg} / \mathrm{m}^{2}$. Caelyx® contains $2 \mathrm{mg} / \mathrm{ml}$ doxorubicin hydrochloride encapsulated in a PEGylated liposomal formulation (16 mg lipid content). Based on established pharmacokinetic data for the clinically-used PEGylated liposomes encapsulating doxorubicin, the time of blood collection (after the completion of infusion that lasted for approximately 90 mins), the majority of injected liposomal nanoparticles are still in blood circulation. ${ }^{[14,42]}$

Paired plasma samples (before and immediately after cycle 1 infusion) were collected into commercially available anticoagulant-treated tubes (K2 EDTA BD Vacutainer®). Plasma was then prepared by inverting 10 times the collection tubes to ensure mixing of blood with EDTA and subsequent centrifugation for 12 minutes at $1300 \mathrm{RCF}$ at $4{ }^{\circ} \mathrm{C}$. Following centrifugation supernatant was immediately collected into labelled Protein LoBind Eppendorf Tubes and samples were maintained on ice while handling.

Separation of corona-coated liposomes from unbound and weakly bound proteins. Corona-coated liposomes were separated form excess plasma proteins by size exclusion chromatography followed by membrane ultrafiltration as we have previously described. ${ }^{[4,5]}$

Ex vivo protein corona formation. To investigate the ex vivo protein corona, Caelyx® liposomes were incubated with plasma samples obtained from the same 6 patients prior to Caelyx $\AA$ infusion and from healthy donors. Considering the impact of the anti-coagulant agent on the formation of protein corona, ${ }^{[43]}$ the same protocol was followed for the preparation of plasma samples, as described above for the in vivo experiment (K2 EDTA BD Vacutainer® tubes, centrifugation for 12 minutes at $1300 \mathrm{RCF}$ at $4{ }^{\circ} \mathrm{C}$ ). The ex vivo protein corona was allowed to form using the same liposome concentration $(0.15 \mathrm{mM})$ as that extracted in $1 \mathrm{~mL}$ of plasma from intravenously injected patients. Liposomes were incubated with plasma for $90 \mathrm{~min}$ at $37^{\circ} \mathrm{C}$ in orbital shaker at $250 \mathrm{rpm}$. The purification of the ex vivo corona-coated liposomes was performed as described above for the in vivo recovered liposomes.

Size and zeta potential measurements using dynamic light scattering (DLS). Liposome size and surface charge were measured using Zetasizer Nano ZS (Malvern, Instruments, UK).

Transmission Electron Microscopy (TEM). Liposomes were stained by uranyl acetate solution $1 \%$ and visualized with transmission electron microscopy (FEI Tecnai 12 BioTwin) before and after their in vivo interaction with plasma proteins. Samples were diluted to $0.5 \mathrm{mM}$ lipid concentration and carbon Film Mesh Copper Grids (CF400-Cu, Electron Microscopy Science) were used.

Cryo-electron Microscopy. EM grids of liposomes were prepared in a FEI Vitrobot using $3 \mu \mathrm{L}$ of sample absorbed to freshly glow-discharged R 3.5/1 Quantifoil grids. Grids were blotted for $4-5 \mathrm{~s}$ in a 95\% humidity chamber before plunge freezing in to liquid ethane. Data were imaged on a Tecnai T20 (FEI) electron microscope operating at $200 \mathrm{keV}$ with a Gatan 626 cryo stage. Images were recorded on a $4 \mathrm{~K}$ Gatan Ultrascan CCD camera under low-dose conditions between 0.5 and $2.0 \mu \mathrm{m}$ defocus and at $3.8 \AA / p i x e l$ and had a maximum electron dose of $<25$ electrons/Å2.

SDS-PAGE electrophoresis. Proteins associated with $0.025 \mu \mathrm{M}$ of liposomes were loaded onto a 4-20\% NOVEX Tris-Glycine Protein Gel (ThermoFisher Scientific). The gel was run until the proteins neared the end of the gel (25-40 minutes at 225V). Staining was performed with Imperial Gel Staining reagent (Sigma Life Science). 
Quantification of adsorbed proteins. Proteins associated with recovered liposomes were quantified by BCA Protein assay kit according to manufacturer's instructions. To make sure that liposomes in solution do not interfere with the absorbance at $562 \mathrm{~nm}$ we measured the absorbance of corona-coated liposomes in HBS buffer and subtract it from the total absorbance, measured when corona-coated liposomes were mixed with the BCA reagent. Lipid concentration was quantified by Stewart assay and $\mathrm{Pb}$ values ( $\mu \mathrm{g}$ of protein/ $\mu \mathrm{M}$ lipid) were then calculated.

Mass Spectrometry. In-gel digestion of corona (40ug) and plasma (5ul) proteins was performed prior to LCMS/MS analysis, as we have previously described.[4-6] Digested samples were analysed by LC-MS/MS using an UltiMate ${ }^{\circledR} 3000$ Rapid Separation LC (RSLC, Dionex Corporation, Sunnyvale, CA) coupled to a Q Exactive $^{\mathrm{TM}}$ Hybrid Quadrupole-Orbitrap ${ }^{\mathrm{TM}}$ (Thermo Fisher Scientific, Waltham, MA) mass spectrometer. Data produced were searched using Mascot (Matrix Science UK), against the SwissProt_2016_04 database with taxonomy of [human] selected.

The Scaffold software (version 4.3.2, Proteome Software Inc.) was used for relative protein quantification based on spectral counting. Protein identifications were accepted if they could be established at greater than $99.0 \%$ probability and contained at least 2 identified peptides. Semi quantitative assessment of the protein amounts was conducted using normalized spectral countings as previously described. ${ }^{[4-6]}$ Heatmaps of Relative Protein Abundance (RPA) values were prepared using Plotly 2.0 software.

Statistical analysis. Statistical analysis of the data was performed using GraphPad Prism software. Oneway analysis of variance (ANOVA) was used and $p$ values $<0.05$ were considered significant.

\section{Data availability}

The authors declare that all relevant data supporting the findings of this study are available within the paper and its supplementary information files.

\section{Author contributions}

M.H. initiated, designed and performed the experiments, analyzed all data and took responsibility for planning and writing the manuscript. S. McA., G.G and C.T. aided in the collection of blood samples. D.K. performed the Mass Spectrometry experiments. IJ.R. and A.C. contributed to the clinical design, provided oversight of the ethical approval process and were responsible for access and storage of the samples in the MCRC Biobank. Z.A-A and M.M provided intellectual input and experimental guidance at the initial stages of the study. K. K. initiated, designed, directed, provided intellectual input throughout the study and contributed to the writing of the manuscript.

\section{Acknowledgements}

This research was partially funded by the Marie Curie Initial Training Network PathChooser (PITN-GA-2013608373). Authors wish to acknowledge Manchester Cancer Research (MCRC) Biobank team (Moghadam Sharzad, Bromley Kevin, Basia Hunt) for their assistance in the collection of blood samples. The authors also would like to thank the Faculty of Life Sciences EM Facility at the University of Manchester and Dr. Marc C. A. Stuart from University of Groningen for their assistance in Electron Microscopy imaging. In addition, Mass Spectrometry Facility staff at the University of Manchester for their support. We would also like to thank the patients for their kind donation of blood samples to support this research. 


\section{References}

[1] M. Hadjidemetriou, K. Kostarelos, Nat Nanotechnol 2017, 12, 288.

[2] T. Cedervall, I. Lynch, S. Lindman, T. Berggard, E. Thulin, H. Nilsson, K. A. Dawson, S. Linse, Proc Natl Acad Sci U S A 2007, 104, 2050.

[3] S. Schottler, G. Becker, S. Winzen, T. Steinbach, K. Mohr, K. Landfester, V. Mailander, F. R. Wurm, Nat Nanotechnol 2016, 11, 372.

[4] M. Hadjidemetriou, Z. Al-Ahmady, M. Mazza, R. F. Collins, K. Dawson, K. Kostarelos, ACS Nano 2015, 9, 8142.

[5] M. Hadjidemetriou, Z. Al-Ahmady, K. Kostarelos, Nanoscale 2016, 8, 6948.

[6] Z. S. Al-Ahmady, M. Hadjidemetriou, J. Gubbins, K. Kostarelos, Journal of Controlled Release 2018, 276, 157.

[7] R. Garcia-Alvarez, M. Hadjidemetriou, A. Sanchez-Iglesias, L. M. Liz-Marzan, K. Kostarelos, Nanoscale 2018.

[8] D. Westmeier, D. Solouk-Saran, C. Vallet, S. Siemer, D. Docter, H. Gotz, L. Mann, A. Hasenberg, A. Hahlbrock, K. Erler, C. Reinhardt, O. Schilling, S. Becker, M. Gunzer, M. Hasenberg, S. K. Knauer, R. H. Stauber, Proc Natl Acad Sci U S A 2018, 115, 7087.

[9] A. Salvati, A. S. Pitek, M. P. Monopoli, K. Prapainop, F. B. Bombelli, D. R. Hristov, P. M. Kelly, C. Aberg, E. Mahon, K. A. Dawson, Nat. Nanotechnol. 2013, 8, 137.

[10] D. Westmeier, R. H. Stauber, D. Docter, Toxicol Appl Pharmacol 2016, 299, 53.

[11] T. M. Allen, P. R. Cullis, Adv Drug Deliv Rev 2013, 65, 36.

[12] Y. Barenholz, J Control Release. 2012, 160, 117.

[13] T. Safra, F. Muggia, S. Jeffers, D. D. Tsao-Wei, S. Groshen, O. Lyass, R. Henderson, G. Berry, A. Gabizon, Annals of Oncology 2000, 11, 1029.

[14] A. Gabizon, H. Shmeeda, Y. Barenholz, Clin Pharmacokinet 2003, 42, 419.

[15] U. Sakulkhu, L. Maurizi, M. Mahmoudi, M. Motazacker, M. Vries, A. Gramoun, M. G. Ollivier Beuzelin, J. P. Vallee, F. Rezaee, H. Hofmann, Nanoscale 2014, 6, 11439.

[16] G. Caracciolo, D. Pozzi, A. L. Capriotti, C. Cavaliere, S. Piovesana, G. La Barbera, A. Amici, A. Lagana, J Mater Chem B 2014, 2, 7419.

[17] S. Wilhelm, A. J. Tavares, Q. Dai, S. Ohta, J. Audet, H. F. Dvorak, W. C. W. Chan, Nat Rev Mater 2016, 1.

[18] D. Docter, D. Westmeier, M. Markiewicz, S. Stolte, S. K. Knauer, R. H. Stauber, Chem Soc Rev 2015, 44, 6094.

[19] J. Wolfram, K. Suri, Y. Yang, J. Shen, C. Celia, M. Fresta, Y. Zhao, H. Shen, M. Ferrari, Colloids Surf B Biointerfaces 2014, 114, 294.

[20] S. Tenzer, D. Docter, J. Kuharev, A. Musyanovych, V. Fetz, R. Hecht, F. Schlenk, D. Fischer, K. Kiouptsi, C. Reinhardt, K. Landfester, H. Schild, M. Maskos, S. K. Knauer, R. H. Stauber, Nat Nanotechnol 2013, 8, 772.

[21] M. Kokkinopoulou, J. Simon, K. Landfester, V. Mailander, I. Lieberwirth, Nanoscale 2017, 9, 8858.

[22] A. Chonn, S. C. Semple, P. R. Cullis, J Biol Chem. 1992, 267, 18759.

[23] A. Chonn, S. C. Semple, P. R. Cullis, Biochim Biophys Acta. 1991, 1070, 215.

[24] S. C. Semple, A. Chonn, P. R. Cullis, Biochemistry 1996, 35, 2521.

[25] E. A. Vogler, Biomaterials 2012, 33, 1201.

[26] F. Chen, G. Wang, J. I. Griffin, B. Brenneman, N. K. Banda, V. M. Holers, D. S. Backos, L. Wu, S. M. Moghimi, D. Simberg, Nat Nanotechnol 2017, 12, 387.

[27] S. Tenzer, D. Docter, J. Kuharev, A. Musyanovych, V. Fetz, R. Hecht, F. Schlenk, D. Fischer, K. Kiouptsi, C. Reinhardt, K. Landfester, H. Schild, M. Maskos, S. K. Knauer, R. H. Stauber, Nat. Nanotechnol. 2013, 8, 772.

[28] Z. Xiong, L. Zhao, F. Wang, J. Zhu, H. Qin, R. Wu, W. Zhang, H. Zou, Chem Commun (Camb) 2012, 48,8138 .

[29] S. Mishra, P. Webster, M. E. Davis, Eur J Cell Biol 2004, 83, 97.

[30] N. Bertrand, P. Grenier, M. Mahmoudi, E. M. Lima, E. A. Appel, F. Dormont, J. M. Lim, R. Karnik, R. Langer, O. C. Farokhzad, Nat Commun 2017, 8, 777.

[31] J. Szebeni, Toxicology 2005, 216, 106.

[32] A. Chanan-Khan, J. Szebeni, S. Savay, L. Liebes, N. M. Rafique, C. R. Alving, F. M. Muggia, Ann Oncol 2003, 14, 1430. 
[33] R. Schiess, B. Wollscheid, R. Aebersold, Mol Oncol 2009, 3, 33.

[34] K. Merrell, K. Southwick, S. W. Graves, M. S. Esplin, N. E. Lewis, C. D. Thulin, J Biomol Tech 2004, 15,238 .

[35] T. Maack, V. Johnson, S. T. Kau, J. Figueiredo, D. Sigulem, Kidney Int 1979, 16, 251.

[36] Y. Vugmeyster, X. Xu, F. P. Theil, L. A. Khawli, M. W. Leach, World J Biol Chem 2012, 3, 73.

[37] A. Luchini, D. H. Geho, B. Bishop, D. Tran, C. Xia, R. L. Dufour, C. D. Jones, V. Espina, A.

Patanarut, W. Zhou, M. M. Ross, A. Tessitore, E. F. Petricoin, 3rd, L. A. Liotta, Nano Lett 2008, 8, 350.

[38] D. Tamburro, C. Fredolini, V. Espina, T. A. Douglas, A. Ranganathan, L. llag, W. Zhou, P. Russo, B. H. Espina, G. Muto, E. F. Petricoin, 3rd, L. A. Liotta, A. Luchini, J Am Chem Soc 2011, 133, 19178.

[39] M. Berle, A. C. Kroksveen, O. A. Haaland, T. T. Aye, J. A. Opsahl, E. Oveland, K. Wester, R. J. Ulvik, C. A. Helland, F. S. Berven, Fluids Barriers CNS 2011, 8, 19.

[40] V. Zavvos, A. T. Buxton, C. Evans, M. Lambie, S. J. Davies, N. Topley, M. Wilkie, A. Summers, P. Brenchley, D. S. Goumenos, T. S. Johnson, Kidney Int 2017, 92, 988.

[41] Y. Wu, Y. H. Z. Feng, R. Shu, Y. Chen, Y. Feng, H. W. Liu, International Journal of Clinical and Experimental Medicine 2016, 9, 15540.

[42] A. Gabizon, R. Catane, B. Uziely, B. Kaufman, T. Safra, R. Cohen, F. Martin, A. Huang, Y. Barenholz, Cancer Res 1994, 54, 987.

[43] M. Lundqvist, C. Augustsson, M. Lilja, K. Lundkvist, B. Dahlback, S. Linse, T. Cedervall, PLoS One 2017, 12, e0175871. 


\section{FIGURE LEGENDS}

Figure 1: The effect of protein corona formation on the physicochemical characteristics and morphology of liposomes. (A) Schematic description of the experimental design. PEGylated, doxorubicinencapsulated liposomes (Caely ${ }^{\circledR}$ ) were infused intravenously in patients with recurrent ovarian carcinoma and subsequently recovered from the blood circulation immediately on completion of their first-ever cycle of Caelyx ${ }^{\circledR}$ treatment. Corona coated liposomes were isolated from blood components and protein corona was quantitatively and qualitatively characterized; (B) Table shows the mean diameter $(\mathrm{nm}), \zeta$-potential $(\mathrm{mV})$ and polydispersity index (PDI) values of bare and corona-coated liposomes recovered from the blood circulation of 6 adenocarcinoma patients. Representative size and $\zeta$-potential distributions of corona-coated liposomes recovered from Patient 1 are also shown; (C) Negative stain TEM and Cryo-EM imaging of liposomes after their I.V injection and recovery from ovarian carcinoma patients. All scale bars are 100nm.

Figure 2: Characterization of in vivo protein corona: (A) The total amount of proteins adsorbed in vivo onto liposomes recovered from the blood circulation of ovarian carcinoma patients. $\mathrm{Pb}$ values ( $\mu \mathrm{g}$ of protein/ $\mu \mathrm{M}$ lipid) represent the mean and standard deviation from 6 patients; (B) Classification of the corona proteins identified according to their molecular mass; (C) Most-abundant proteins (top-20) identified in the protein corona of PEGylated doxorubicin-encapsulated liposomes by LC-MS/MS. Relative protein abundance (RPA) values represent the average and standard deviation from 6 ovarian carcinoma patients; (D) The relative percentage of immunoglobulins, lipoproteins and complement proteins identified in the protein corona. The percentage of relative protein abundance (\%RPA) for each protein class represents the average from 6 ovarian carcinoma patients.

Figure 3: Blood-circulation proteome analysis: (A) Imperial stained SDS-PAGE gel of corona proteins associated with liposomes in the blood circulation of 6 ovarian carcinoma patients and plasma control; (B) Venn diagram reports the number of unique and common proteins between corona and plasma proteins and their respective overlaps as identified by LC-MS/MS. Proteins were identified in at least one of the six plasma and/or corona samples; (C) Heatmap of RPA (\%) of corona and plasma proteins, as identified by LC-MS/MS. Only proteins with RPA $>1 \%$ on at least one of the samples are shown. Protein-rows are sorted according to the RPA\% values (from highest to lowest) of the first sample (plasma, patient 1). The full list of proteins identified and their respective accession numbers are shown in Table S3 and Table S4. 


\section{FIGURE 1}

\section{A}

B
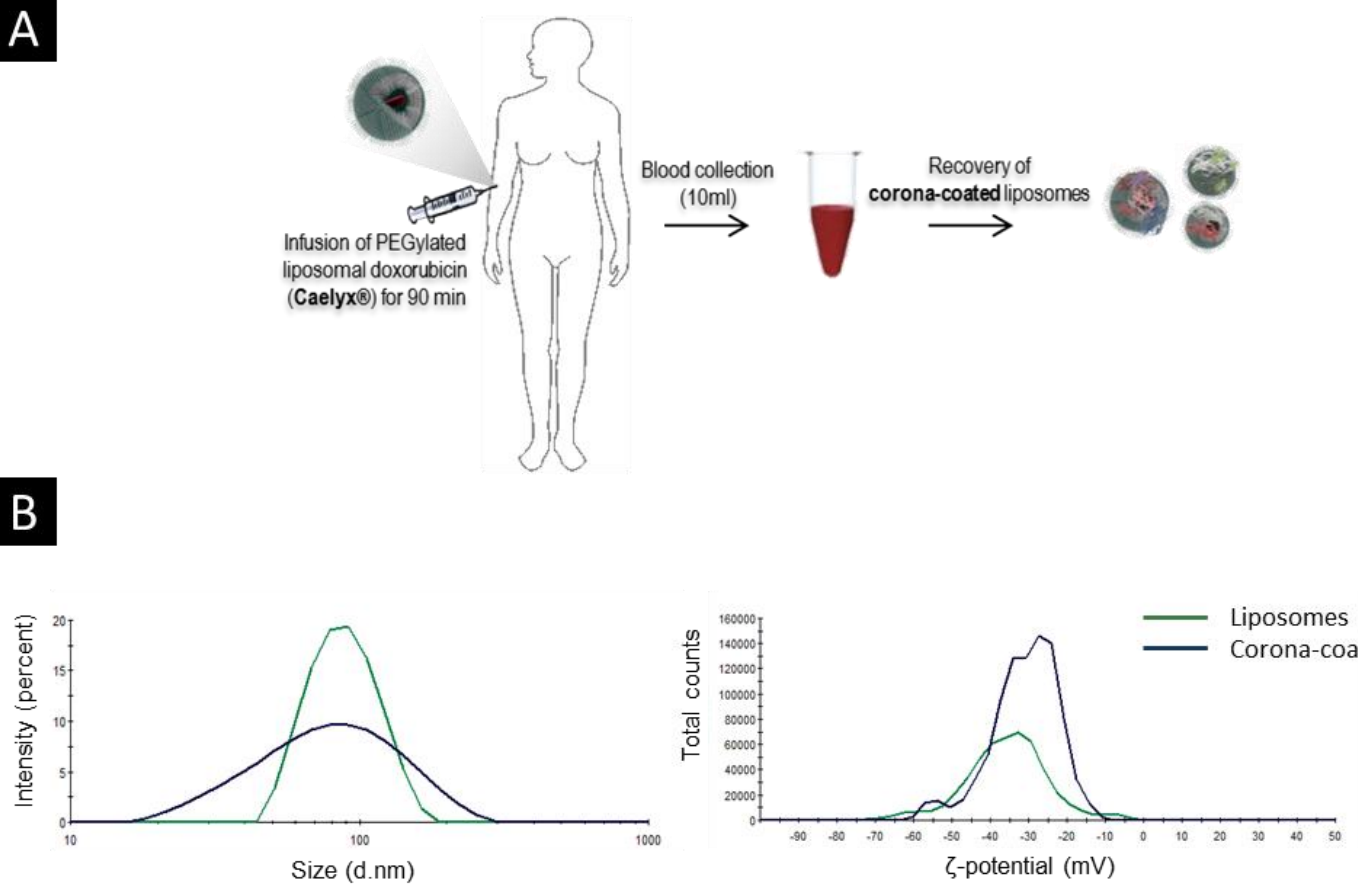

\begin{tabular}{l|ccc} 
& $\begin{array}{c}\text { Mean hydrodynamic } \\
\text { diameter }(\mathbf{n m})\end{array}$ & $\begin{array}{c}\text { \%-potential } \\
(\mathbf{m V})\end{array}$ & PDI \\
\hline $\begin{array}{l}\text { Liposomes-Caelyx } \\
\text { [HSPC/CHOL/mPEG2000-DSPE(56.2:38.555.3)] }\end{array}$ & $82.63 \pm 1.391$ & $-33.8 \pm 1.74$ & $0.087 \pm 0.023$ \\
Corona-coated liposomes (Patient1) & $72.81 \pm 1.120$ & $-30.7 \pm 1.87$ & $0.275 \pm 0.021$ \\
Corona-coated liposomes (Patient2) & $89.56 \pm 1.662$ & $-36.2 \pm 0.85$ & $0.243 \pm 0.011$ \\
Corona-coated liposomes (Patient3) & $80.80 \pm 0.782$ & $-33.0 \pm 2.04$ & $0.234 \pm 0.006$ \\
Corona-coated liposomes (Patient4) & $86.95 \pm 3.469$ & $-37.5 \pm 0.76$ & $0.228 \pm 0.008$ \\
Corona-coated liposomes (Patient5) & $79.78 \pm 0.550$ & $-30.8 \pm 0.06$ & $0.274 \pm 0.006$ \\
Corona-coated liposomes (Patient6) & $98.28 \pm 2.012$ & $-39.8 \pm 2.80$ & $0.229 \pm 0.002$
\end{tabular}

\section{C}

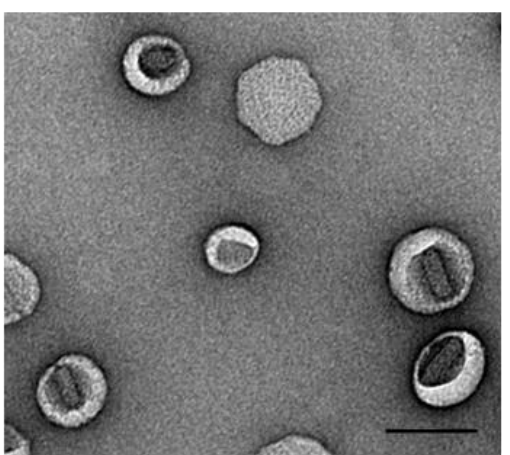

Liposomes $\left(\right.$ Caelyx $^{\circledast}$ ) by TEM

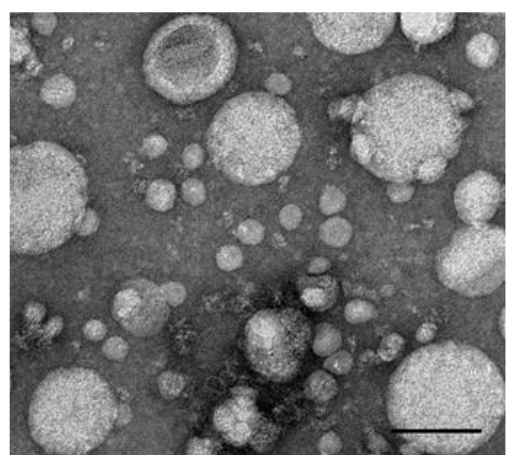

Liposome: in vivo corona by TEM

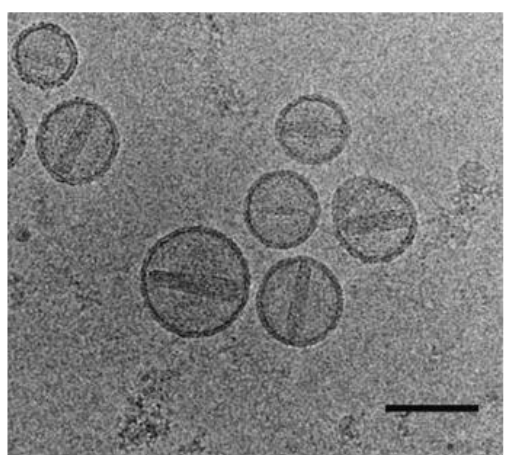

Liposome: in vivo corona by cryo-EM 
FIGURE 2

A

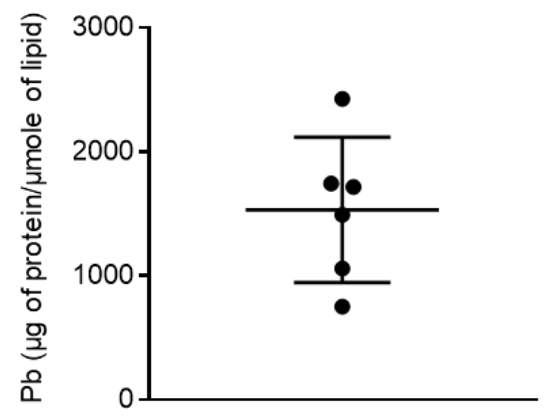

B

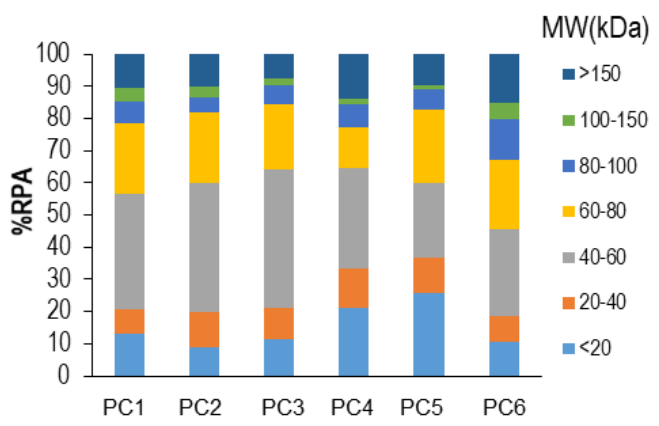

C

TOP 20 IDENTIFIED PROTENS

Full-length cDNA clone CSODD006YL02 of Homo sapiens (human)

Ig mu chain $\mathrm{C}$ region (A0A087X2C0)

Ig mu chain $\mathrm{C}$ region (A0A087WY J9)

Alpha-2-macroglobulin

Fibrinogen beta chain

Fibrinogen alpha chain

Apolipoprotein B (Including $\mathrm{Ag}(\aleph$ antigen)

Serum albumin

APOB protein

Protein IGHV3-72

C4b-binding protein alpha chain

Fibrinogen gamma chain

Haptoglobin (Fragment)

Haptoglobin

IGH protein

Ig gamma-1 chain $\mathrm{C}$ region

Uncharacterized protein (A8K008)

Uncharacterized protein (Q6N089)

IgG $\mathrm{H}$ chain

Actin, cytoplasmic 2

\section{RPA\%}

$7.99 \pm 2.48$

$5.70 \pm 1.81$

$4.56 \pm 2.83$

$3.48 \pm 1.02$

$3.45 \pm 1.19$

$2.98 \pm 0.67$

$2.64 \pm 1.44$

$2.47 \pm 2.06$

$2.22 \pm 2.10$

$1.91 \pm 0.58$

$1.74 \pm 0.59$

$1.59 \pm 0.53$

$1.35 \pm 0.34$

$1.35 \pm 0.43$

$1.14 \pm 0.64$

$1.12 \pm 0.59$

$1.11 \pm 0.59$

$1.05 \pm 0.60$

$1.04 \pm 0.64$

$1.04 \pm 0.60$
Immunoglobulins

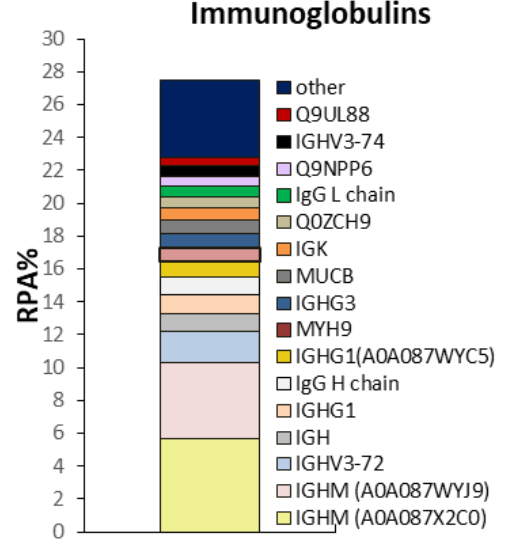

Lipoproteins

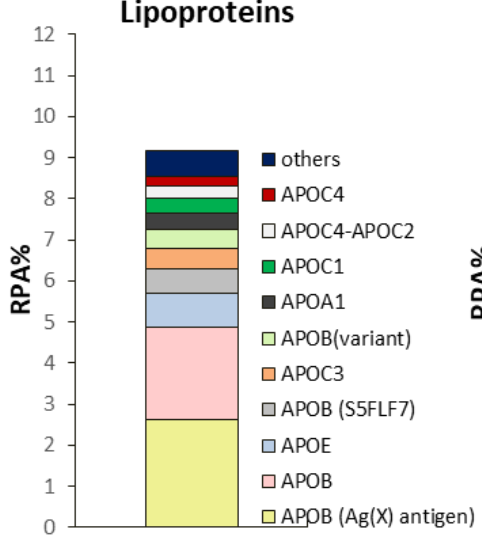

\section{Complement}

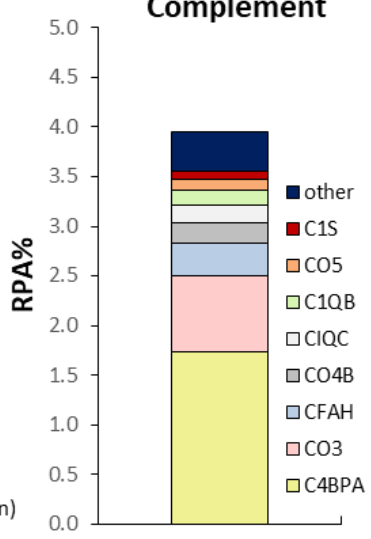




\section{FIGURE 3}

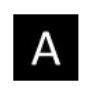

12

13

14

15

16

17

18

19

20

21

22

23

24

25

26

27

28

29

30

31

32

33

34

35

36

37

38

39

40

41

42

43

44

45

46

47

48

49

50

51

52

53

54

55

56

57

58

59

60

61

62

63

64

65
B

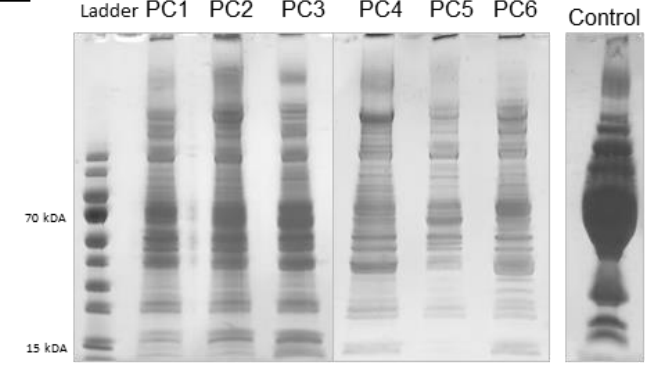

\section{Plasma Corona}

$\begin{array}{lll}70 & 188 & 257\end{array}$

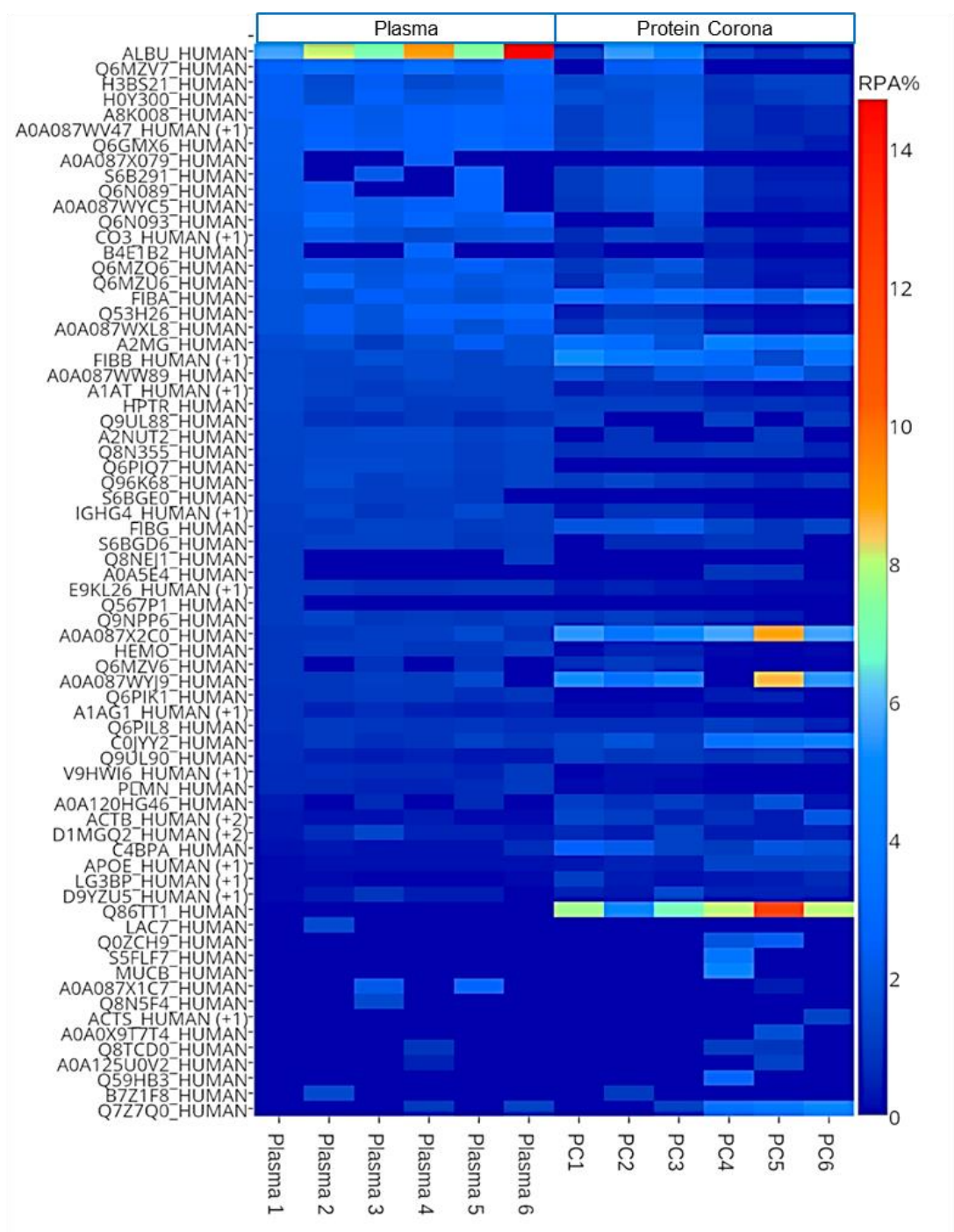




\title{
SUPPORTING INFORMATION
}

\author{
Supporting Figure 1
}

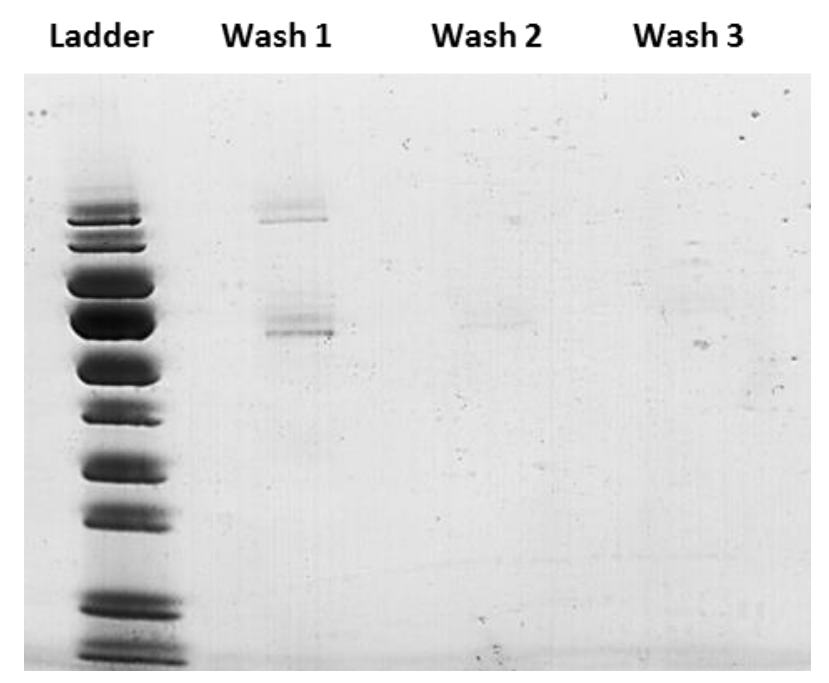

Figure S1: Separation of protein corona coated liposomes from unbound proteins. Corona-coated liposomes were separated from excess plasma proteins by size exclusion chromatography followed by membrane ultrafiltration. Immediately after plasma preparation, plasma samples were loaded onto a Sepharose CL-4B (SIGMA-ALDRICH) column $(15 \times 1.5 \mathrm{~cm})$ equilibrated with HBS. Fractions containing liposomes (fractions 4,5\&6) were then pooled together and concentrated to $500 \mu$ l by centrifugation using Vivaspin 6 column (10000 MWCO, Sartorious, Fisher Scientific) at 3000rpm. Vivaspin 500 centrifugal concentrator (1000 000 MWCO, Sartorious, Fisher Scientific) was then used at $3000 \mathrm{rpm}$, to further concentrate the samples to $100 \mu \mathrm{l}$ and to ensure separation of protein-coated nanoparticles from the remaining large unbound proteins. This procedure results in the filtration of unbound proteins while coronacoated liposomes because of their higher MW remain at the top of the membrane. Imperial stained SDSPAGE shows that any remaining proteins in Chromatographic Fractions 4,5 and 6 were removed to the filtrate of Viva Spin (wash1) and no proteins remained at the top of the membrane (column residue). Column residue was washed 2 more time with HBS to ensure complete purification. 


\section{Supporting Figure 2}

\begin{tabular}{l|ccc} 
Ex vivo & $\begin{array}{c}\text { Mean hydrodynamic } \\
\text { diameter }(\mathbf{n m})\end{array}$ & $\begin{array}{c}\text { Z-potential } \\
(\mathbf{m V})\end{array}$ & PDI \\
\hline Corona-coated liposomes (Patient1) & $73.80 \pm 1.518$ & $-32.1 \pm 3.50$ & $0.161 \pm 0.005$ \\
Corona-coated liposomes (Patient2) & $72.28 \pm 1.095$ & $-30.2 \pm 2.97$ & $0.227 \pm 0.006$ \\
Corona-coated liposomes (Patient3) & $75.05 \pm 1.500$ & $-37.2 \pm 1.80$ & $0.149 \pm 0.005$ \\
Corona-coated liposomes (Patient4) & $97.88 \pm 1.980$ & $-27.8 \pm 3.91$ & $0.244 \pm 0.015$ \\
Corona-coated liposomes (Patient5) & $86.61 \pm 1.393$ & $-28.7 \pm 0.40$ & $0.238 \pm 0.006$ \\
Corona-coated liposomes (Patient6) & $98.03 \pm 1.812$ & $-40.9 \pm 2.51$ & $0.237 \pm 0.007$
\end{tabular}
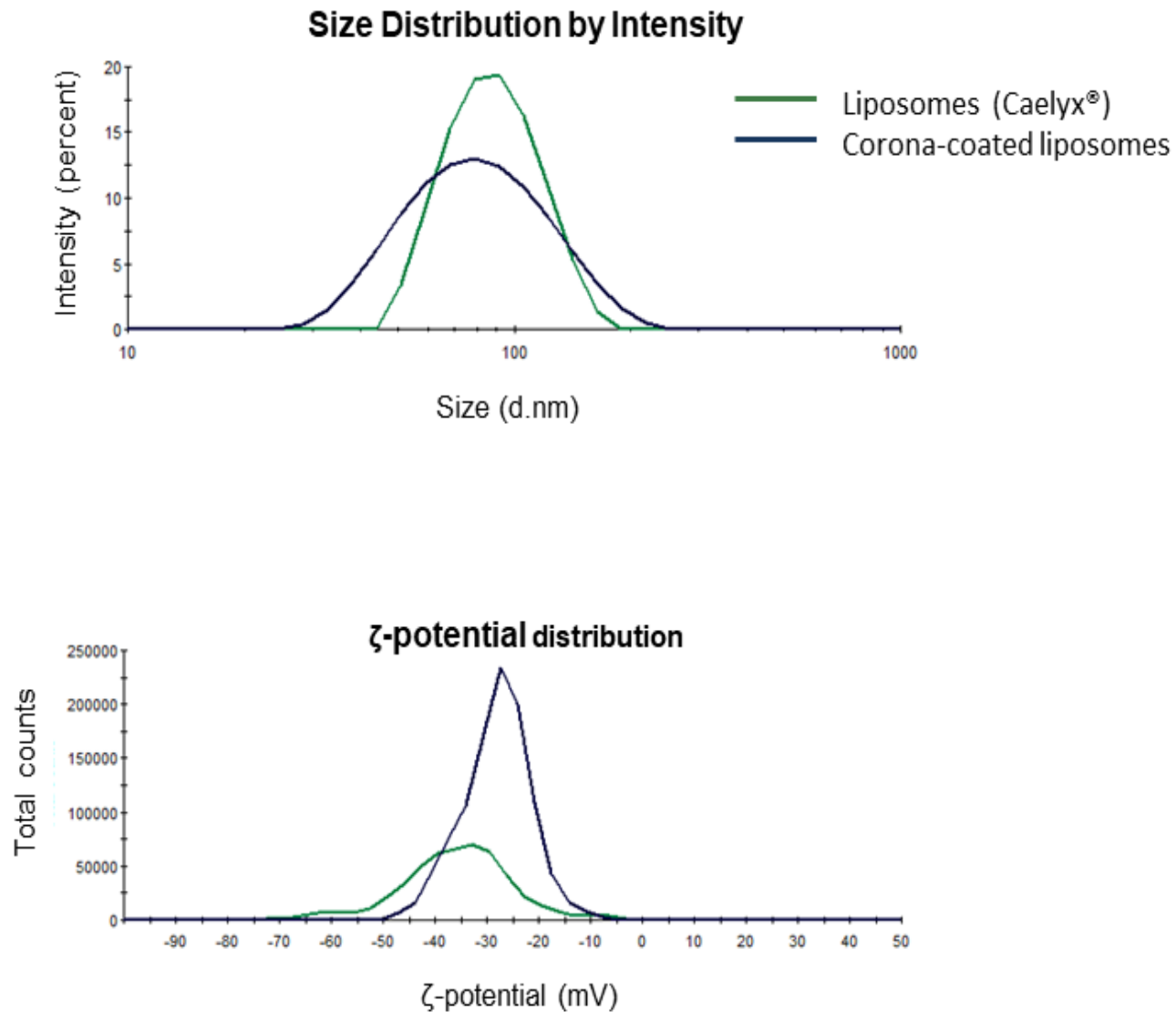

Figure S2: The effect of the ex vivo formed protein corona on the physicochemical characteristics of liposomes. Mean diameter $(\mathrm{nm}), \zeta$-potential $(\mathrm{mV})$ and polydispersity index (PDI) values and representative size and $\zeta$-potential distributions of bare and corona-coated liposomes incubated ex vivo with plasma samples obtained from 6 ovarian adenocarcinoma patient. 


\section{Supporting Figure 3}

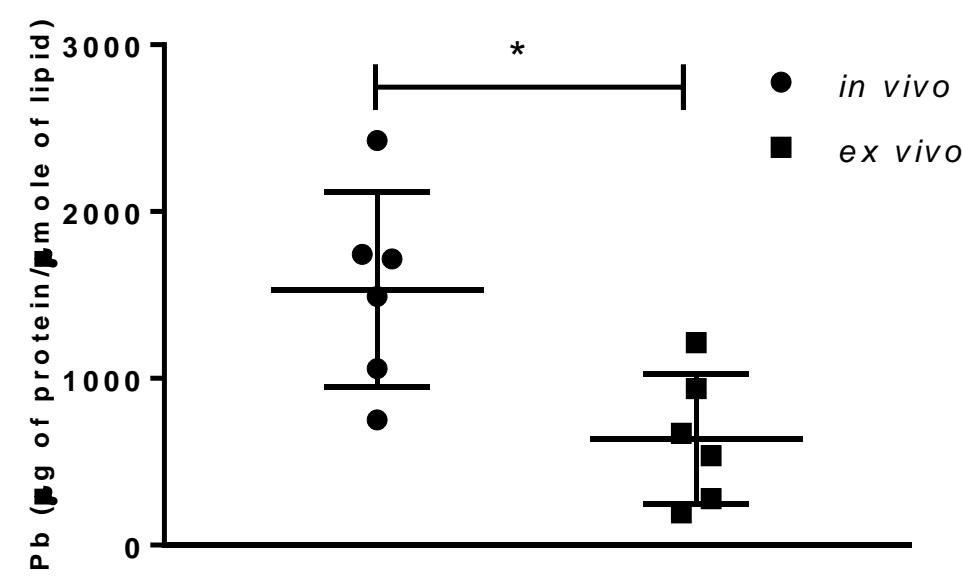

Figure S3: Comparison of in vivo vs. ex vivo protein coronas. The total amount of proteins adsorbed onto liposomes in vivo after recovery from the blood circulation of ovarian carcinoma patients and after their ex vivo incubation with plasma samples obtained from the same patients before the infusion of Caelyx ${ }^{\circledR}$. $\mathrm{Pb}$ values ( $\mu \mathrm{g}$ of protein $/ \mu \mathrm{M}$ lipid) represent the mean and standard deviation from 6 patients; ${ }^{* *}$ indicates $p<0.01$ $(p=0.0086)$; * indicates $p<0.05(p=0.0111)$. 


\section{Supporting Figure 4}

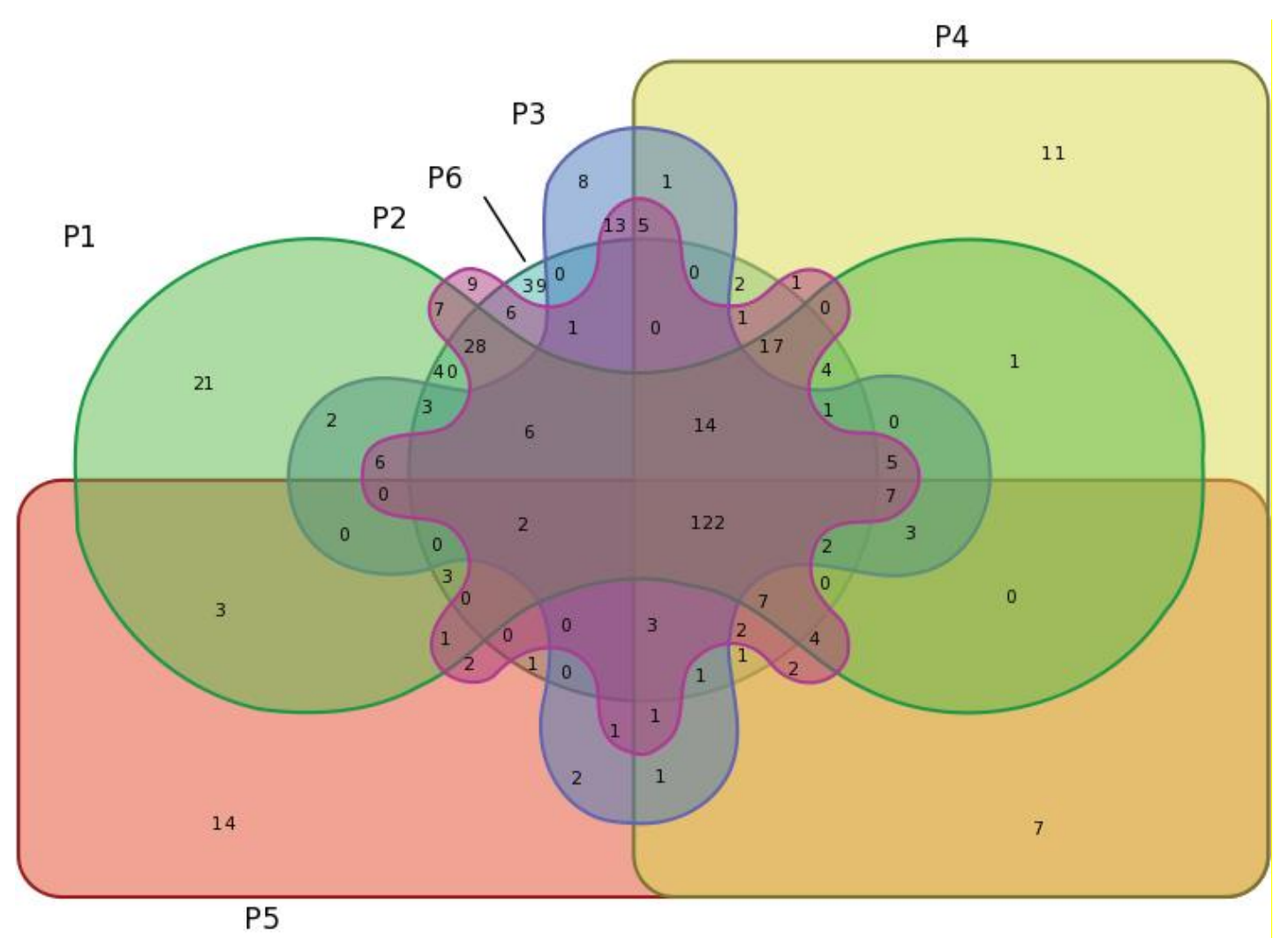

Figure S4: Characterisation of the in vivo protein corona: Venn diagram reports the number of unique and common proteins between the liposomal coronas formed in 6 ovarian carcinoma patients and their respective overlaps as identified by LC-MS/MS. The full list of common proteins identified in the liposomal protein corona in all 6 ovarian carcinoma patients are shown in Tables S5. 


\section{Supporting Figure 5}

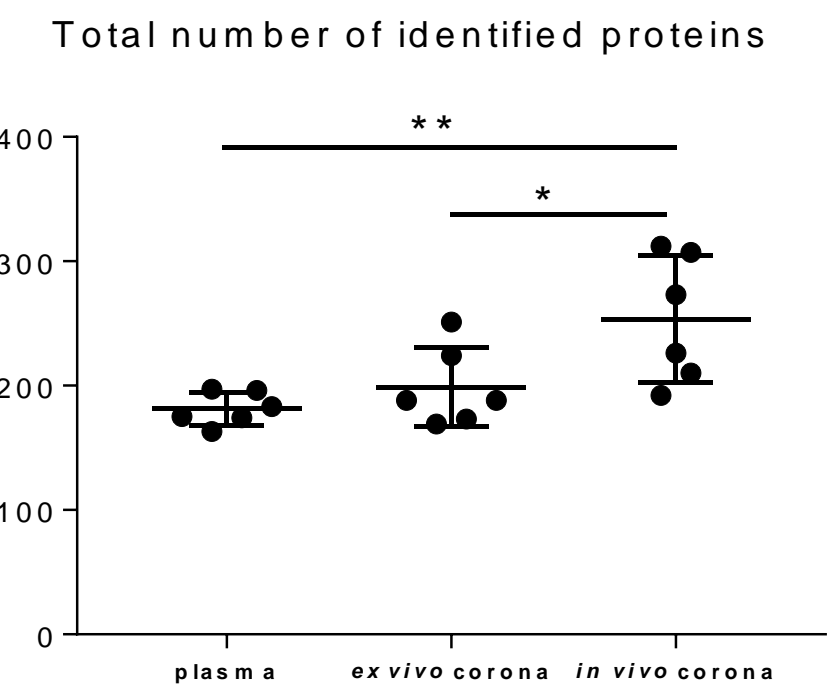

Figure S5: Blood-circulation proteome analysis. Total number of proteins identified by mass spectrometry analysis of plasma samples obtained from ovarian carcinoma patients and corona samples formed ex vivo and in vivo. Values represent the mean and standard deviation from 6 patients; ${ }^{*}$ indicates $p<0.05$ $(p=0.0459)$ and ${ }^{* *}$ indicates $p<0.01(p=0.0087)$. 


\section{Supporting Table 1}

Table S1: Patient cohort clinical and basic blood characteristics.

\begin{tabular}{|l|l|}
\hline Age (yrs) & Median 71 (56-77) \\
\hline Prior lines of chemotherapy & Median 2 (range 2-3) \\
\hline Histological subtype & $\begin{array}{l}\text { High grade serous carcinoma- 5 } \\
\text { Moderately differentiated carcinoma NOS-1 }\end{array}$ \\
\hline FIGO stage at diagnosis & $\begin{array}{l}\text { IIIC-5 } \\
\text { IVA-1 }\end{array}$ \\
\hline Baseline CA125 (at commencement of Caelyx) (U/ml) & Median 665 (103-3081) \\
\hline Albumin (at commencement of Caelyx) (g/L) & Median 41 (30-44) \\
\hline Total protein (at commencement of Caelyx) (g/L) & Median 68 (56-72) \\
\hline
\end{tabular}




\section{Supporting Table 2}

Table S2: Most-abundant proteins (top-20) identified by LC-MS/MS in the protein corona of PEGylated liposomal doxorubicin, recovered from 6 different ovarian carcinoma patients.

\begin{tabular}{l|l|l|l|}
\multicolumn{2}{|c|}{ Patient 1 } & \\
\cline { 2 - 4 } & \multicolumn{2}{|c|}{ Patient 2 } \\
\cline { 2 - 4 } $\mathbf{1}$ & Identified Proteins & RPA\% & Identified Proteins \\
\cline { 2 - 4 } $\mathbf{2}$ & Full-length cDNA clone CS0DD006YL02 & 7.68 & Serum albumin \\
$\mathbf{3}$ & Ig mu chain C region(A0A087X2C0) & 5.42 & Full-length cDNA clone CS0DD006YL02 \\
$\mathbf{4}$ & Fibrinogen C region(A0A087WYJ9) & 5.17 & Fibrinogen beta chain \\
$\mathbf{5}$ & Alpha-2-macroglobulin & 5.17 & Ig mu chain C region(A0A087X2C0) \\
$\mathbf{6}$ & Fibrinogen alpha chain & 3.49 & Ig mu chain C region(A0A087WYJ9) \\
$\mathbf{7}$ & C4b-binding protein alpha chain & 3.25 & Alpha-2-macroglobulin \\
$\mathbf{8}$ & Fibrinogen gamma chain & 2.55 & Fibrinogen alpha chain \\
$\mathbf{9}$ & Protein IGHV3-72 & 1.92 & Putative uncharacterized protein DKFZp686C11235 \\
$\mathbf{1 0}$ & Haptoglobin & 1.84 & C4b-binding protein alpha chain \\
$\mathbf{1 1}$ & Haptoglobin (Fragment) & 1.65 & Fibrinogen gamma chain \\
$\mathbf{1 2}$ & Actin, cytoplasmic 2 & 1.41 & Putative uncharacterized protein DKFZp686C15213 \\
$\mathbf{1 3}$ & Serum albumin & 1.40 & Apolipoprotein B (Including Ag(X) antigen) \\
$\mathbf{1 4}$ & Apolipoprotein B (Including Ag(X) antigen) & 1.36 & Ig gamma-3 chain C region \\
$\mathbf{1 5}$ & Myosin-reactive immunoglobulin heavy chain variable region & 1.31 & IGH protein \\
$\mathbf{1 6}$ & IGH protein & 1.18 & Haptoglobin (Fragment) \\
$\mathbf{1 7}$ & Myosin-reactive immunoglobulin heavy chain variable region (Fragment) & 1.16 & Ig gamma-1 chain C region \\
$\mathbf{1 8}$ & GCT-A10 heavy chain variable region (Fragment) & 1.15 & Uncharacterized protein (Q6N089) \\
$\mathbf{1 9}$ & Uncharacterized protein (A8K008) & 1.14 & IgG H chain \\
$\mathbf{2 0}$ & Lectin galactoside-binding soluble 3 binding protein isoform 1 (Fragment) & 1.13 & Uncharacterized protein(A8K008) \\
& & 1.11 & Haptoglobin \\
\hline
\end{tabular}




\section{Supporting Table 3}

Table S3: Characterization of in vivo protein corona. Full list of all the proteins identified in the liposomal protein corona recovered from 6 ovarian carcinoma patients (across all samples).

RPA\% (P3)
RPA\% (P4)
RPA\% $\quad$ RPA $\% \quad$ Average $\begin{array}{llll}\text { (P5) } & \text { (P6) } & \text { RPA\% } & \text { STV }\end{array}$

$\begin{array}{lll}66 & 5.174 & 3.402\end{array}$

6.908

$7.987 \quad 12.443$

7.997 $7.985 \quad 2.476$ Uncharacterized protein OS=Homo sapiens

Q6GMX6_HUMAN

$163 \quad 3.494$

3.119

4.812

$5.693 \quad 8.994$

5.756

$5.701 \quad 1.813$

A0A087WV47_HUMAN (+1)

A8K008_HUMAN

\section{Q6N089_HUMAN}

S6B291_HUMAN

ACTG_HUMAN (+1)

$56 \quad 5.166$

3.952

4.659

$0.000 \quad 8.654$

$\begin{array}{lllllllll}95 & 3.253 & 2.704 & 3.282 & 2.766 & 1.964 & 3.927 & 2.983 & 0.666\end{array}$

$\begin{array}{lllllllll}516 & 1.309 & 1.744 & 1.096 & 3.368 & 3.794 & 4.518 & 2.638 & 1.439\end{array}$

A0A087WYC5_HUMAN

$\begin{array}{lllllllll}69 & 1.359 & 5.469 & 4.718 & 1.320 & 0.692 & 1.252 & 2.468 & 2.062\end{array}$

A0A120HG46_HUMAN

\begin{tabular}{lllllllll}
92 & 0.000 & 0.000 & 1.205 & 3.421 & 3.786 & 4.924 & 2.223 & 2.102 \\
\hline
\end{tabular}

$\begin{array}{lllllllll}11 & 1.840 & 1.120 & 1.904 & 2.112 & 2.853 & 1.602 & 1.905 & 0.575\end{array}$

Q96K68HUMAN

HPTR_HUMAN

$\begin{array}{lll}67 & 2.553 \quad 2.098\end{array}$

1.179

$0.979 \quad 1.953$

$\begin{array}{llllll}52 & 1.916 & 1.913 & 2.224 & 1.445 & 0.757\end{array}$

1.657

$1.736 \quad 0.589$

$\begin{array}{lllllllll}25 & 1.407 & 1.661 & 1.771 & 0.820 & 1.238 & 1.222 & 1.353 & 0.343\end{array}$

$\begin{array}{lllllllll}49 & 1.653 & 1.490 & 1.923 & 0.734 & 1.056 & 1.223 & 1.347 & 0.429\end{array}$

$\begin{array}{lllllllll}51 & 1.160 & 1.673 & 2.109 & 0.813 & 0.650 & 0.438 & 1.140 & 0.643\end{array}$




\begin{tabular}{|c|c|c|c|c|c|c|c|c|c|}
\hline S6BGD6_HUMAN & 25 & 0.490 & 0.621 & 0.628 & 0.893 & 0.760 & 0.353 & 0.624 & 0.191 \\
\hline Q9NPP6_HUMAN & 45 & 0.781 & 1.105 & 0.804 & 0.617 & 0.413 & 0.000 & 0.620 & 0.380 \\
\hline A2KBC6_HUMAN & 25 & 0.725 & 0.456 & 0.666 & 0.747 & 0.831 & 0.235 & 0.610 & 0.223 \\
\hline S5FLF7_HUMAN & 10 & 0.000 & 0.000 & 0.000 & 3.644 & 0.000 & 0.000 & 0.607 & 1.488 \\
\hline A2NUT2_HUMAN & 25 & 0.000 & 0.803 & 0.819 & 1.020 & 0.973 & 0.000 & 0.602 & 0.474 \\
\hline A0A0K2BMD8_HUMAN (+2) & 15 & 0.604 & 0.365 & 1.174 & 0.395 & 0.442 & 0.548 & 0.588 & 0.301 \\
\hline A0A0B4J1X5_HUMAN & 13 & 0.656 & 0.421 & 0.586 & 0.596 & 0.918 & 0.316 & 0.582 & 0.207 \\
\hline AOA0S2Z4K3_HUMAN (+2) & 75 & 0.838 & 0.560 & 0.374 & 0.425 & 0.542 & 0.705 & 0.574 & 0.173 \\
\hline Q9UL88_HUMAN & 14 & 1.180 & 0.000 & 0.000 & 1.171 & 0.000 & 0.965 & 0.553 & 0.610 \\
\hline A0A0S2Z3Y1_HUMAN (+1) & 65 & 1.115 & 0.414 & 0.242 & 0.343 & 0.354 & 0.560 & 0.505 & 0.317 \\
\hline A3KPE2_HUMAN (+2) & 11 & 0.145 & 0.166 & 0.173 & 0.828 & 0.643 & 1.068 & 0.504 & 0.399 \\
\hline B4E1B2_HUMAN & 75 & 0.376 & 1.028 & 0.920 & 0.352 & 0.088 & 0.235 & 0.500 & 0.383 \\
\hline B2R950_HUMAN (+1) & 164 & 0.481 & 0.545 & 0.360 & 0.592 & 0.466 & 0.516 & 0.493 & 0.079 \\
\hline TSP1_HUMAN & 129 & 0.785 & 0.350 & 0.244 & 0.300 & 0.363 & 0.852 & 0.482 & 0.264 \\
\hline AOAOC4DH38_HUMAN & 13 & 0.451 & 0.421 & 0.439 & 0.420 & 0.680 & 0.407 & 0.470 & 0.104 \\
\hline A0A024QZN4_HUMAN (+2) & 117 & 0.610 & 0.378 & 0.228 & 0.358 & 0.227 & 0.944 & 0.458 & 0.277 \\
\hline A0A024R462_HUMAN & 259 & 0.485 & 0.384 & 0.351 & 0.378 & 0.469 & 0.665 & 0.455 & 0.116 \\
\hline Q59HB3_HUMAN & 184 & 0.000 & 0.000 & 0.000 & 2.681 & 0.000 & 0.000 & 0.447 & 1.094 \\
\hline H6VRF8_HUMAN (+3) & 66 & 0.452 & 0.512 & 0.346 & 0.545 & 0.342 & 0.418 & 0.436 & 0.084 \\
\hline FLNA_HUMAN & 281 & 0.595 & 0.351 & 0.212 & 0.311 & 0.160 & 0.889 & 0.420 & 0.275 \\
\hline Q6MZV6_HUMAN & 52 & 0.738 & 1.000 & 0.741 & 0.000 & 0.000 & 0.000 & 0.413 & 0.463 \\
\hline HV306_HUMAN & 12 & 0.355 & 0.266 & 0.238 & 0.493 & 0.774 & 0.343 & 0.412 & 0.198 \\
\hline A0A125QYY9_HUMAN & 14 & 0.381 & 0.554 & 0.000 & 0.423 & 0.726 & 0.378 & 0.410 & 0.241 \\
\hline A1AT_HUMAN (+1) & 47 & 0.442 & 0.738 & 0.638 & 0.291 & 0.150 & 0.200 & 0.410 & 0.239 \\
\hline TLN1_HUMAN & 270 & 0.610 & 0.370 & 0.213 & 0.317 & 0.170 & 0.775 & 0.409 & 0.236 \\
\hline A0A024R694_HUMAN (+1) & 103 & 0.672 & 0.443 & 0.194 & 0.301 & 0.146 & 0.673 & 0.405 & 0.231 \\
\hline A0A024R3E3_HUMAN (+1) & 31 & 0.395 & 0.442 & 0.461 & 0.544 & 0.242 & 0.303 & 0.398 & 0.110 \\
\hline Q53H26_HUMAN & 77 & 0.374 & 1.037 & 0.940 & 0.000 & 0.000 & 0.000 & 0.392 & 0.485 \\
\hline VWF_HUMAN & 309 & 0.760 & 0.347 & 0.421 & 0.124 & 0.132 & 0.546 & 0.388 & 0.246 \\
\hline A0A0G2JPD4_HUMAN (+1) & 36 & 0.340 & 0.837 & 0.820 & 0.316 & 0.000 & 0.000 & 0.386 & 0.373 \\
\hline L8E853_HUMAN & 298 & 0.771 & 0.358 & 0.441 & 0.000 & 0.138 & 0.592 & 0.383 & 0.284 \\
\hline QOKKI6_HUMAN (+1) & 24 & 0.000 & 0.000 & 0.000 & 1.234 & 1.032 & 0.000 & 0.378 & 0.588 \\
\hline A2J1N6_HUMAN & 13 & 0.000 & 0.000 & 0.586 & 0.876 & 0.748 & 0.000 & 0.368 & 0.414 \\
\hline F13A_HUMAN & 83 & 0.558 & 0.341 & 0.247 & 0.181 & 0.117 & 0.722 & 0.361 & 0.234 \\
\hline A0A024R0T8_HUMAN (+2) & 9 & 0.000 & 0.355 & 0.000 & 0.708 & 0.540 & 0.522 & 0.354 & 0.296 \\
\hline
\end{tabular}


\begin{tabular}{l|l|l}
4 & & SV=1 \\
\hline 5 & & Rheumatoid factor RF-ET6 (Fragment)
\end{tabular}

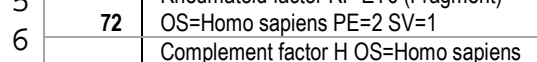

\begin{tabular}{l|l|l}
7 & 73 & $\mathrm{GN}=\mathrm{CFH} P E=1 \mathrm{SV}=4$ \\
8 & & Keratin, type I cytoskeletal $10 \mathrm{OS}=$ Homo
\end{tabular}

\begin{tabular}{l|l|l}
8 & $\mathbf{2}$ & $\begin{array}{l}\text { Keratin, type I cytoskeletal } 10 \mathrm{OS}=\mathrm{Hom} \\
\text { sapiens } \mathrm{GN}=\mathrm{KRT} 10 \mathrm{PE}=1 \mathrm{SV}=6\end{array}$ \\
\hline 9
\end{tabular}

$9 \quad 74$ Protein APOC4-APOC2 OS=Homo

1075 sapiens GN=APOC4-APOC2 PE=1 SV=1 MS-D3 heavy chain variable region

6 (Fragment) OS=Homo sapiens $\mathrm{PE}=2 \mathrm{SV}=1$ Ig heavy chain $V$-III region WEA OS=Homo

1377 sapiens $P E=1 \mathrm{SV}=1$

Uncharacterized protein OS=Homo

78 sapiens $\mathrm{PE}=2 \mathrm{SV}=1$

Keratin, type I cytoskeletal 9 OS=Homo

cDNA FLJ75066, highly similar to Homo

sapiens complement component $1, r$

subcomponent (C1R), mRNA OS=Homo

80 sapiens $P E=2 \mathrm{SV}=1$

Epididymis tissue protein Li 173 OS=Homo

81 sapiens $\mathrm{GN}=\mathrm{SERPING1} \mathrm{PE}=2 \mathrm{SV}=1$

Myosin, heavy polypeptide 9 , non-muscle,

isoform CRA_a OS=Homo sapiens

$82 \mathrm{GN}=\mathrm{MYH} 9 \mathrm{PE}=4 \mathrm{SV}=1$

Cryocrystalglobulin $\mathrm{CC} 1$ heavy chain

variable region (Fragment) OS=Homo

83 sapiens $P E=2 \mathrm{SV}=1$

Putative uncharacterized protein

DKFZp686104196 (Fragment) OS=Homo

sapiens GN=DKFZp686I04196 PE=2

Heat shock cognate $71 \mathrm{kDa}$ protein

OS=Homo sapiens $\mathrm{GN}=\mathrm{HSPA} 8 \mathrm{PE}=1$

$85 \quad S V=1$

Apolipoprotein C-IV OS=Homo sapiens

$86 \mathrm{GN}=\mathrm{APOC} 4 \mathrm{PE}=2 \mathrm{SV}=1$

Hemopexin OS=Homo sapiens GN=HPX

$87 \quad \mathrm{PE}=1 \mathrm{SV}=2$

Epididymis secretory protein Li 52

$\mathrm{OS}=$ Homo sapiens $\mathrm{GN}=\mathrm{HEL}-\mathrm{S}-52 \mathrm{PE}=2$

Actin, alpha skeletal muscle OS=Homo

89 sapiens $\mathrm{GN}=\mathrm{ACTA} 1 \mathrm{PE}=1 \mathrm{SV}=1$

Keratin, type II cytoskeletal 2 epidermal

OS=Homo sapiens $\mathrm{GN}=\mathrm{KRT} 2 \mathrm{PE}=1 \mathrm{SV}=2$

Putative uncharacterized protein

DKFZp686M08189 OS=Homo sapiens

$91 \mathrm{GN}=\mathrm{DKFZ}$ 6869M08189 PE=2 SV=1

Fermitin family homolog 3 OS=Homo

92 sapiens $\mathrm{GN}=\mathrm{FERMT} 3 \mathrm{PE}=1 \mathrm{SV}=1$

Complement C4-B OS=Homo sapiens

$93 \mathrm{GN}=\mathrm{C} 4 \mathrm{~B} \quad \mathrm{PE}=1 \mathrm{SV}=2$

Pyruvate kinase PKM OS=Homo sapiens

$4 \quad G N=P K M \quad P E=1 \quad S V=4$

GCT-A1 heavy chain variable region

A2J1N5_HUMAN

CFAH_HUMAN

K1C10_HUMAN

K7ER74_HUMAN

AOA0X9T7T4_HUMAN

HV302_HUMAN

A0A5E4_HUMAN

K1C9_HUMAN

10 0.479 0.319 0.238 0.410 0.619 0.000 $0.344 \quad 0.214$

PEBLM2000267, highly similar to Tubulin alpha-ubiquitous chain OS=Homo sapiens

$97 \quad \mathrm{PE}=2 \mathrm{SV}=1$ cDNA FLJ56821, highly similar to Interalpha-trypsin inhibitor heavy chain $\mathrm{H} 1$

A8K5J8 HUMAN (+1)

E9KL26_HUMAN (+1)

A0A024R1N1_HUMAN (+1)

B1N7B6_HUMAN

Q6N093_HUMAN

$\begin{array}{lll}46 & 0.000 \quad 0.000\end{array}$

$71 \quad 0.330$

0.296

$\begin{array}{lll}15 & 0.000 & 0.091\end{array}$

0.000

$52 \quad 0.143$

HEMO_HUMAN

V9HWG7_HUMAN (+1)

ACTS_HUMAN (+1)

$\begin{array}{lll}66 & 0.347 & 0.228\end{array}$

$\begin{array}{lll}42 & 0.000 & 0.000\end{array}$

K22E_HUMAN

$65 \quad 0.295$

0.225

$\begin{array}{lll}52 & 0.697 & 0.000\end{array}$

Q6MZX9_HUMAN

URP2_HUMAN

$\begin{array}{lll}76 & 0.273 & 0.150\end{array}$

$193 \quad 0.177 \quad 0.331$

$0.331 \quad 0.289$

$\begin{array}{lll}58 & 0.340 & 0.252\end{array}$

$\begin{array}{lll}14 & 0.000 \quad 0.000\end{array}$

0.148

0.000

$86 \quad 0.30$

GELS_HUMAN

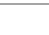

14

$0.304 \quad 0.202$

0.144

0.115

0.11

.110
0.000

0.067

$0.000 \quad 1.315$

0.217 0.21

$0.154 \quad 0.294$

$0.294 \quad 0.109$ $0.587 \quad 0.000$

0.000

0.2

$214 \quad 0.333$

Ig heavy chain V-III region GAL OS=Homo

sapiens $P E=1 \mathrm{SV}=1$

Immunglobulin heavy chain variable region

101 (Fragment) OS=Homo sapiens $P E=4$ SV=1

Complement component $1, q$

subcomponent, C chain, isoform CRA_a

102 OS=Homo sapiens $\mathrm{GN}=\mathrm{C} 1 \mathrm{C}$ PE=4 SV=1

Tubulin alpha-4A chain OS=Homo sapiens

103 GN=TUBA4A PE=1 SV=1
B3KPS3 HUMAN (+2)

46

B7Z549_HUMAN (+1)

HV320_HUMAN

QOZCH6_HUMAN

A0A024RAA7_HUMAN (+1)

TBA4A_HUMAN
CD5L_HUMAN

\begin{tabular}{lllllllll|}
46 & 0.359 & 0.139 & 0.197 & 0.139 & 0.000 & 0.307 & 0.190 & 0.129 \\
76 & 0.154 & 0.312 & 0.194 & 0.204 & 0.081 & 0.178 & 0.187 & 0.075 \\
38 & 0.210 & 0.216 & 0.301 & 0.156 & 0.163 & 0.077 & 0.187 & 0.075 \\
13 & 0.000 & 0.491 & 0.000 & 0.000 & 0.612 & 0.000 & 0.184 & 0.287 \\
14 & 0.228 & 0.163 & 0.170 & 0.163 & 0.253 & 0.126 & 0.184 & 0.047 \\
26 & 0.164 & 0.193 & 0.146 & 0.193 & 0.153 & 0.226 & 0.179 & 0.030 \\
50 & 0.341 & 0.091 & 0.171 & 0.109 & 0.062 & 0.294 & 0.178 & 0.115
\end{tabular}


4 \begin{tabular}{l|l}
\hline & Actinin alpha 4 isoform 1 (Fragment)
\end{tabular}

$5 \quad$ OS=Homo sapiens $\mathrm{GN}=\mathrm{ACTN} 4 \mathrm{PE}=2$

CDNA FLJ53487, highly similar to

AOAOS2Z3G9 HUMAN (+1)

105

0.416

0.230

0.000

$0.108 \quad 0.000$

0.308

$0.177 \quad 0.170$

Coagulation factor XIII A chain ( $E C$

$8 \quad 105 \quad 2.3 .2 .13) \mathrm{OS}=$ Homo sapiens $\mathrm{PE}=2 \mathrm{SV}=1$ Profilin-1 OS=Homo sapiens GN=PFN1

B4E2L8_HUMAN

$\begin{array}{lllllllll}70 & 0.000 & 0.274 & 0.000 & 0.169 & 0.000 & 0.579 & 0.170 & 0.230\end{array}$

106

$P E=1 S V=2$

(Fragment) OS=Homo sapiens $\mathrm{PE}=4 \mathrm{SV}=1$

PROF1 HUMAN

$15 \quad 0.213 \quad 0.274$

0.000

$0.121 \quad 0.000$

0.392

$0.167 \quad 0.156$

QOZCF6_HUMAN

$15 \quad 0.213$

0.122

0.127

0.243

0.295

0.000

$0.167 \quad 0.106$

highly similar to ALPHA-1-

ANTICHYMOTRYPSIN OS=Homo sapiens

$108 \quad \mathrm{PE}=2 \mathrm{SV}=1$

B3KS79_HUMAN

$51 \quad 0.146$

0.340

0.289

0.089

0.061

0.069

$0.166 \quad 0.120$

CDNA FLJ53025, highly similar to

Complement C4-B OS=Homo sapiens

$109 P E=2 S V=1$

B7Z1F8_HUMAN

$\begin{array}{lll}30 & 0.000 & 0.989\end{array}$

0.000

0.000

0.000

0.000

$0.165 \quad 0.404$

110 sapiens GN=JCHAIN PE=1 SV=4

$78 \mathrm{kDa}$ glucose-regulated protein

IGJ_HUMAN

$18 \quad 0.17$

OS=Homo sapiens $\mathrm{GN}=\mathrm{HSPA} 5 \mathrm{PE}=1$

$111 \quad \mathrm{SV}=2$

Inter-alpha (Globulin) inhibitor $\mathrm{H} 2$

GRP78_HUMAN (+1)

$\begin{array}{lll}72 & 0.259 & 0.165\end{array}$

0.079

0.202

0.270

0.131

$0.164 \quad 0.067$

subcomponent, $B$ chain, isoform CRA_a

117 OS=Homo sapiens $G N=C 1 Q B P E=4$ SV=1 Beta 5 -tubulin OS=Homo sapiens

118 GN=TUBB PE=2 $S V=1$ $\lg \mathrm{H}$ chain $\mathrm{OS}=$ Homo sapiens $\mathrm{PE}=2$

Rheumatoid factor RF-IP12 (Fragment)

120 OS=Homo sapiens $\mathrm{PE}=2 \mathrm{SV}=1$ Protein IGHV3-73 OS=Homo sapiens

$121 \mathrm{GN}=$ IGHV3-73 $P E=1 \mathrm{SV}=1$

Lipoprotein, $L p(A)$ OS=Homo sapiens

122 GN=LPA PE=1 SV=1

Keratin, type I cytoskeletal 16 OS=Homo

123 sapiens $\mathrm{GN}=\mathrm{KRT} 16 \mathrm{PE}=1 \mathrm{SV}=4$ Heat shock protein HSP 90-alpha

OS=Homo sapiens $\mathrm{GN}=\mathrm{HSP} 90 \mathrm{AA} 1 \mathrm{PE}=1$

124 SV=5

Putative uncharacterized protein

DKFZp686C02220 (Fragment) OS=Homo

sapiens GN=DKFZp686C02220 PE=2

A2RTY6_HUMAN (+3)

$106 \quad 0.131$

Q6PIK1_HUMAN

$\begin{array}{lll}25 & 0.000 & 0.000\end{array}$

0.033 $0.076 \quad 0.031$

0.375 $0.156 \quad 0.139$ MOES_HUMAN (+1)

$\begin{array}{lll}68 & 0.212 & 0.121\end{array}$

B4E2F9_HUMAN

$\begin{array}{lll}83 & 0.424 & 0.000\end{array}$

0.166

0.16

APOM_HUMAN

$\begin{array}{lll}21 & 0.076 & 0.152\end{array}$

0.000

0.437

$\begin{array}{lll}0.077 & 0.121 & 0.065\end{array}$

0.155

$0.156 \quad 0.053$

Platelet factor 4 OS=Homo sapiens

$26 \quad \mathrm{GN}=\mathrm{PF} 4 \mathrm{PE}=1 \mathrm{SV}=2$

Apolipoprotein D (Fragment) OS=Homo

sapiens $\mathrm{GN}=\mathrm{APOD} P E=1 \mathrm{SV}=1$

Keratin, type I cytoskeletal 14 OS=Homo

128 sapiens $\mathrm{GN}=\mathrm{KRT} 14 \mathrm{PE}=1 \mathrm{SV}=4$

Inter-alpha (Globulin) inhibitor H4 (Plasma

Kallikrein-sensitive glycoprotein)

129 OS=Homo sapiens GN=ITIH4 PE=2 SV=1 Tubulin beta- 1 chain OS=Homo sapiens

$130 \mathrm{GN}=T \mathrm{TUB} 1 \mathrm{PE}=1 \mathrm{SV}=1$ Protein SAA2-SAA4 OS=Homo sapiens

$\begin{array}{lllllllllll}\text { A0A024RAB9_HUMAN (+3) } & 27 & 0.138 & 0.152 & 0.071 & 0.236 & 0.098 & 0.196 & 0.149 & 0.061\end{array}$

\author{
Q5SU16_HUMAN (+1)
}

S6B2A6_HUMAN

$50 \quad 0.394 \quad 0.082$

0.067

0.082

$0.082 \quad 0.018$

A2J1M8_HUMAN

$\begin{array}{lll}32 & 0.000 & 0.000\end{array}$

0.684

$0.185 \quad 0.000$

0.235

$0.146 \quad 0.142$

A0A0G2JN55_HUMAN

$\begin{array}{lll}11 & 0.242 & 0.166\end{array}$

0.000

0.16

Q1HP67_HUMAN

$\begin{array}{lll}9 & 0.000 & 0.000\end{array}$

0.000

0.166

0.281

0.000

$0.145 \quad 0.274$

K1C16_HUMAN

$227 \quad 0.005$

0.296

0.342

0.014

0.835

0.000

$0.139 \quad 0.341$

$51 \quad 0.084$

0.134

0.065

0.20

HS90A_HUMAN (+1)

$\begin{array}{lll}85 & 0.176 & 0.134\end{array}$

0.084 0.07

Q6N091_HUMAN

$54 \quad 0.000 \quad 0.465$

0.000

$0.287 \quad 0.000$

0.000

$0.125 \quad 0.202$

PLF4_HUMAN

C9JF17_HUMAN

$\begin{array}{lll}11 & 0.194 & 0.124\end{array}$

$\begin{array}{lll}0.000 & 0.166 & 0.000\end{array}$

$\begin{array}{lll}24 & 0.000 & 0.000\end{array}$

0.000

$0.247 \quad 0.203$

0.267

$\begin{array}{ll}0.125 & 0.108\end{array}$

K1C14_HUMAN

$\begin{array}{lll}52 & 0.113 & 0.123\end{array}$

0.000

0.2

B2RMS9_HUMAN (+1)

$\begin{array}{lll}103 & 0.093 & 0.191\end{array}$

0.254 $0.097 \quad 0.026$ 0.136 0.12

$0.124 \quad 0.139$

$\mathrm{GN}=\mathrm{SAA} 2-\mathrm{SAA} 4 \mathrm{PE}=4 \mathrm{SV}=1$

TBB1_HUMAN

$\begin{array}{lll}50 & 0.277 & 0.100\end{array}$ 0.076

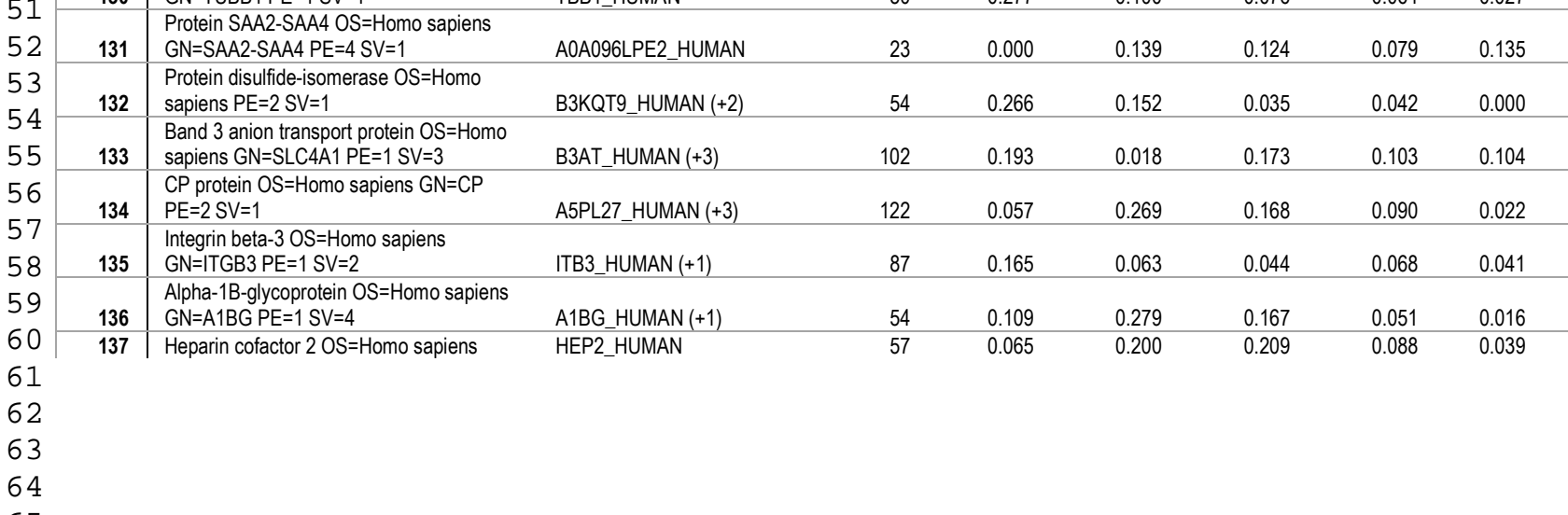

0.124

$\begin{array}{ll}0.064 & 0.027\end{array}$

0.051 $0.119 \quad 0.087$

AOA096LPE2_HUMAN

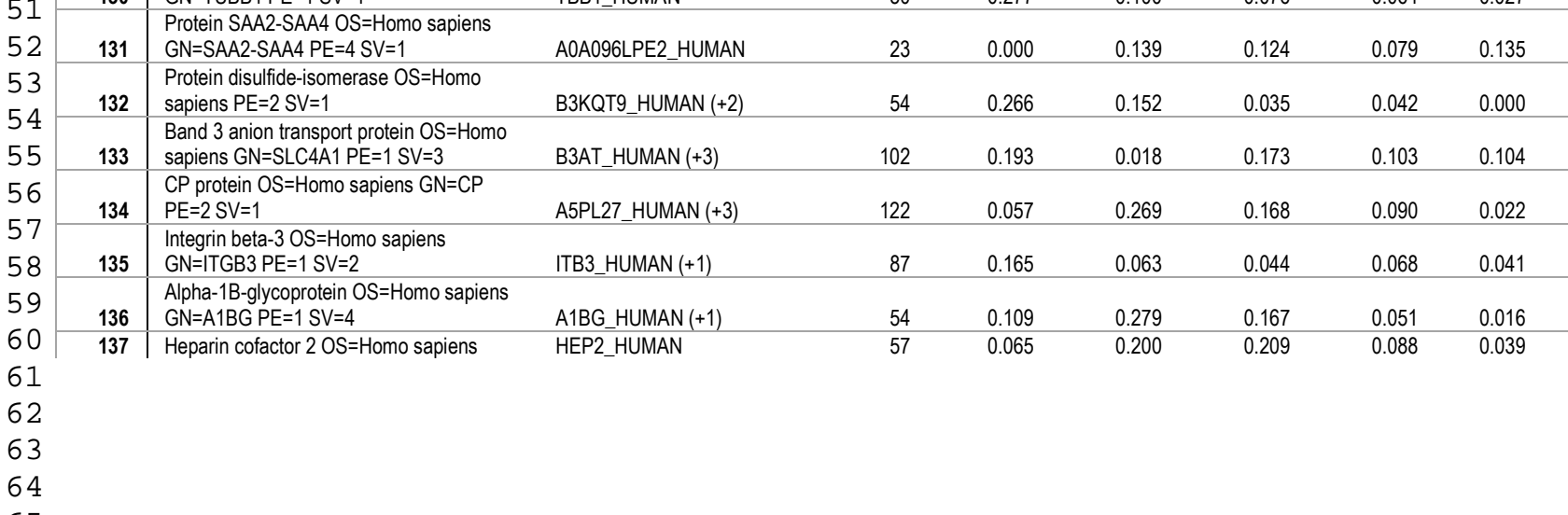

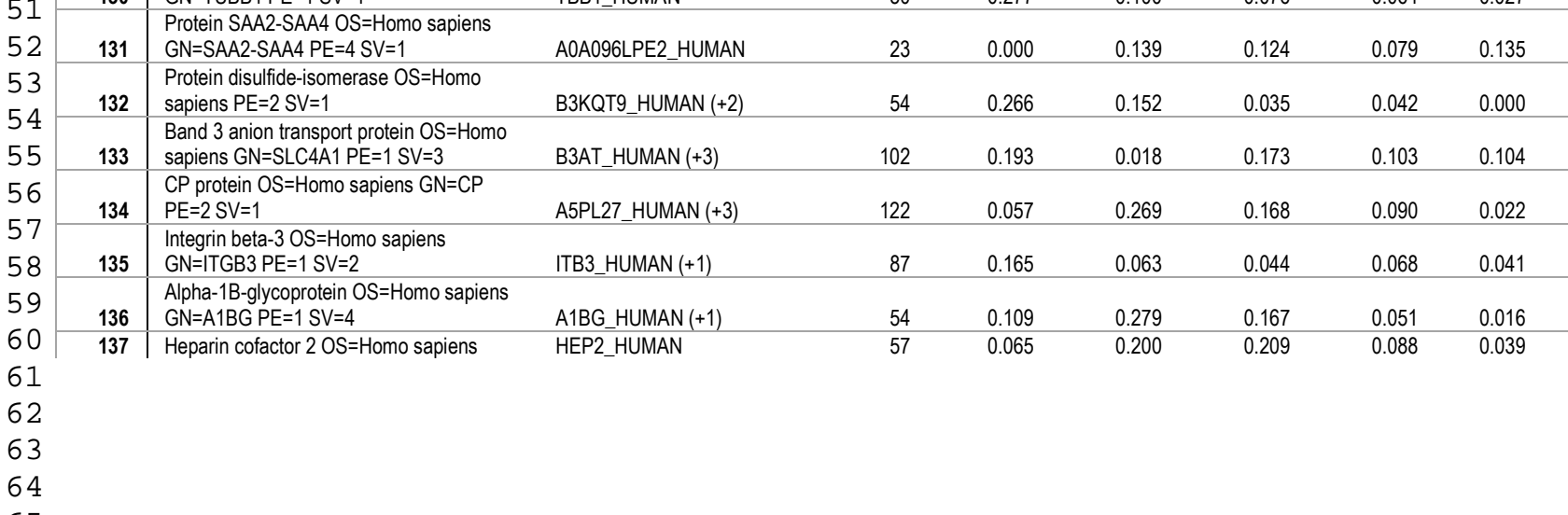

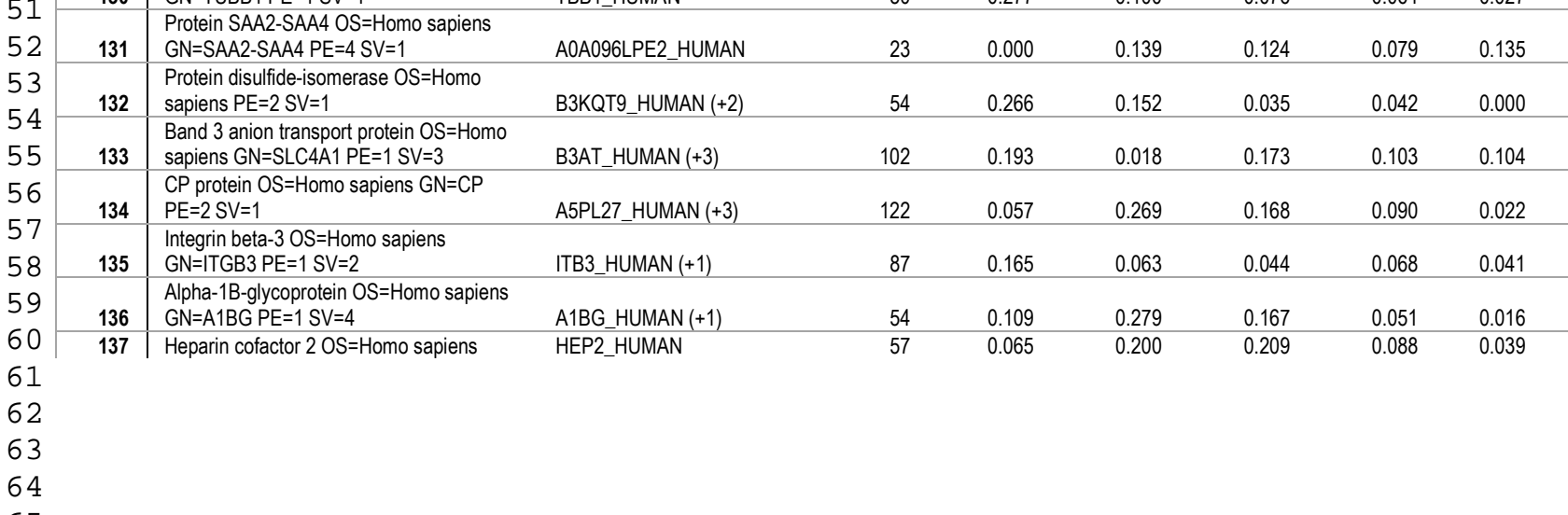

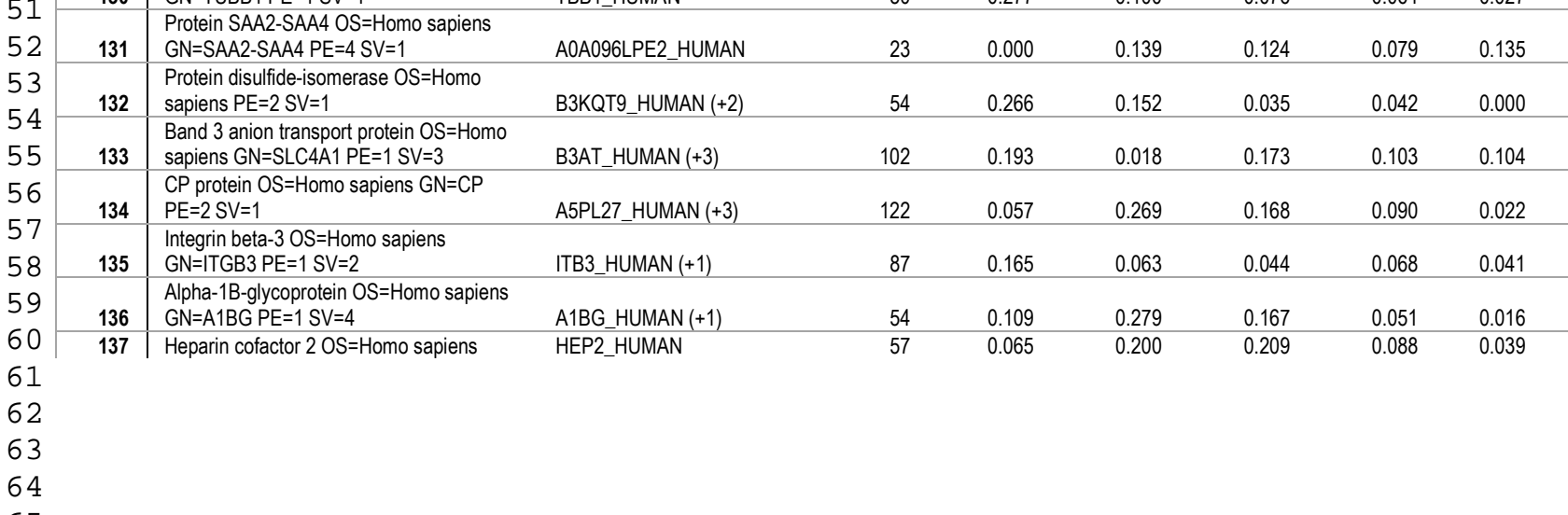


$4 \quad$\begin{tabular}{l|l}
\hline & GN=SERPIND1 PE=1 SV=3
\end{tabular}

5 Ig heavy chain V-III region CAM OS=Homo

0.182

$\begin{array}{lll}0.180 & 0.075 & 0.035\end{array}$

0.044 $0.102 \quad 0.065$

$\mathrm{PE}=1 \mathrm{SV}=1$

Adenylyl cyclase-associated protein

145 OS=Homo sapiens GN=CAP1 PE=3 SV=1

Complement $\mathrm{C}$ 1s subcomponent

146 OS=Homo sapiens $\mathrm{GN}=\mathrm{C} 1 \mathrm{~S} P \mathrm{PE}=1 \mathrm{SV}=1$

Complement C4-A OS=Homo sapiens

$147 \mathrm{GN}=\mathrm{C} 4 \mathrm{~A} P \mathrm{PE}=1 \mathrm{SV}=1$

CLUS_HUMAN

$\begin{array}{lll}92 & 0.139 & 0.099\end{array}$

0.047

0.05

$\begin{array}{lll}13 & 0.082 & 0.140\end{array}$

0.000

$0.280 \quad 0.102$

0.243

$0.101 \quad 0.080$

Glyceraldehyde-3-phosphate

dehydrogenase $\mathrm{OS}=\mathrm{Homo}$ sapiens

ropomyosin alpha-4 chain OS=Homo

sapiens $\mathrm{PE}=2 \mathrm{SV}=1$

Heat shock protein $90 \mathrm{kDa}$ alpha

(Cytosolic), class $\mathrm{B}$ member 1 , isoform

CRA_a OS=Homo sapiens

$150 \quad \mathrm{GN}=\mathrm{HSP} 90 \mathrm{AB} 1 \mathrm{PE}=3 \mathrm{SV}=1$ C4b-binding protein beta chain OS=Homo

151 sapiens $\mathrm{GN}=\mathrm{C} 4 \mathrm{BPB} \mathrm{PE}=1 \mathrm{SV}=1$ Anti-Influenza $A$ hemagglutinin heavy chain variable region (Fragment)

152 OS=Homo sapiens PE=2 SV=1

Integrin alpha-llb OS=Homo sapiens

$153 \mathrm{GN}=$ ITGA2B $P E=1 \mathrm{SV}=3$

Vitronectin OS=Homo sapiens GN=VTN

$154 \quad \mathrm{PE}=4 \mathrm{SV}=1$

Keratin, type II cytoskeletal 5 OS=Homo

155 sapiens $\mathrm{GN}=\mathrm{KRT} 5 \mathrm{PE}=1 \mathrm{SV}=3$

Ig heavy chain $V$-III region GA OS=Homo

156 sapiens $\mathrm{PE}=1 \mathrm{SV}=1$

GCT-A2 heavy chain variable region

157 (Fragment) OS=Homo sapiens PE=2 SV=1 IgGFc-binding protein OS=Homo sapiens

$158 \mathrm{GN}=\mathrm{FCGBP} P E=1 \mathrm{SV}=1$

Plasminogen OS=Homo sapiens $\mathrm{GN}=\mathrm{PLG}$

$159 \quad \mathrm{PE}=1 \mathrm{SV}=2$

Ficolin (Collagen/fibrinogen domain

containing) 3 (Hakata antigen), isoform

CRA_b OS=Homo sapiens $\mathrm{GN}=\mathrm{FCN} 3$

$160 \quad P E=2 S V=1$

Vitamin D-binding protein OS=Homo

161 sapiens $\mathrm{GN}=\mathrm{GC} \mathrm{PE}=1 \mathrm{SV}=1$

D6RF35_HUMAN

A0A024RD80_HUMAN (+2)

$\begin{array}{lll}52 & 0.113 & 0.08\end{array}$

D3DPU2_HUMAN

$52 \quad 0.246$

0.123 0.073

C1S_HUMAN

$\begin{array}{lll}77 & 0.090 & 0.207\end{array}$

0.073

0.10

A0A0G2JPRO_HUMAN

193

G3P_HUMAN (+1)

$36 \quad 0.133$

0.165

0.066

$\begin{array}{ll}0.063 & 0.037\end{array}$

0.098

$0.094 \quad 0.048$

B4DVY2_HUMAN (+2)

$\begin{array}{lll}26 & 0.184 & 0.088\end{array}$

0.037

$0.088 \quad 0.000$

0.158

$0.092 \quad 0.070$

C4BPB_HUMAN

$\begin{array}{lll}83 & 0.122 & 0.088\end{array}$

0.063

0.055

0.048

0.177

$0.092 \quad 0.050$

G1FM90_HUMAN

$\begin{array}{lll}28 & 0.171 & 0.098\end{array}$

$0.051 \quad 0.081$

ITA2B_HUMAN

$\begin{array}{lll}15 & 0.213 & 0.152\end{array}$

0.000

0.000

0.079

0.063

$0.091 \quad 0.043$

D9ZGG2_HUMAN (+1)

$\begin{array}{lll}113 & 0.132 & 0.040\end{array}$

0.055

$\begin{array}{lll}0.060 & 0.047 & 0.198\end{array}$

$0.090 \quad 0.101$

K2C5_HUMAN

$54 \quad 0.05$

0.127

0.203

0.03

HV308_HUMAN

$\begin{array}{lll}62 & 0.112 & 0.088\end{array}$

$0.069 \quad 0.1$

$\begin{array}{lll}13 & 0.205 & 0.000\end{array}$

0.000

0.175

0.050

$14 \quad 0.000$

A0A087WXI2_HUMAN (+1)

$\begin{array}{lll}445 & 0.152 & 0.084\end{array}$

0.000

0.09

PLMN_HUMAN

$91 \quad 0.076$

0.216

0.062

0.136

0.052

$P E=1 S V=1$

cDNA FLJ53075, highly similar to

Kininogen-1 OS=Homo sapiens $\mathrm{PE}=2$

50

51

163 SV $=1$

14-3-3 protein zeta/delta OS=Homo

164 sapiens $\mathrm{GN}=\mathrm{YWHAZ} \mathrm{PE}=1 \mathrm{SV}=1$

Transitional endoplasmic reticulum

ATPase OS=Homo sapiens $\mathrm{GN}=\mathrm{VCP}$

$165 \quad P E=1 S V=4$

Uncharacterized protein (Fragment)

166 OS=Homo sapiens $P E=1 \quad S V=1$

cDNA FLJ41552 fis, clone

COLON2004478, highly similar to Protein

Tro alpha1 $\mathrm{H}$,myeloma OS=Homo sapiens

$167 \quad \mathrm{PE}=2 \mathrm{SV}=1$

Platelet glycoprotein $V$ OS=Homo sapiens

$168 \mathrm{GN}=\mathrm{GP5} \mathrm{PE}=1 \mathrm{SV}=1$

Q6UXM4_HUMAN (+1)

$\begin{array}{lll}32 & 0.117 & 0.057\end{array}$

0.074

$0.114 \quad 0.055$

$53 \quad 0.030$

CALR_HUMAN (+2)

$\begin{array}{lll}48 & 0.144 & 0.105\end{array}$

0.225

$0.026 \quad 0.000$

0.092

0.08

$0.085 \quad 0.027$

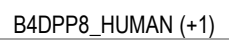

B4DPP8_HUMAN (+1)

$\begin{array}{lll}46 & 0.046 & 0.149\end{array}$

$0.040 \quad 0.028$

$0.028 \quad 0.000$

1433Z_HUMAN (+1)

$\begin{array}{lll}28 & 0.057 & 0.098\end{array}$

0.207

$0.050 \quad 0.000$

$0.000 \quad 0.147$

TERA_HUMAN (+1)

$89 \quad 0.138$

0.067

0.034

0.081

$0.081 \quad 0.032$

0.000

$13 \quad 0.205$

A0A0J9YX35_HUMAN

0.016

0.04

0.000

0.00

Q6ZW64_HUMAN

$\begin{array}{lll}53 & 0.000 \quad 0.000\end{array}$

0.000

0.000

0.434

0.000

$0.072 \quad 0.177$

GPV_HUMAN

$61 \quad 0.052$

0.120

0.000

0.03

0.02

0.202


$4 \quad$ Platelet basic protein OS=Homo sapiens

\begin{tabular}{ll|l}
5 & 169 & GN=PPBP PE=1 SV=3 \\
6 & & Proteoglycan 4, isoform CRA_a OS=Homo
\end{tabular}

\begin{tabular}{l|l|l}
6 & 170 & $\begin{array}{l}\text { Proteoglycan 4, isoform CRA_a OS=Homo } \\
\text { sapiens GN=PRG4 PE=4 SV=1 }\end{array}$ \\
\hline 7 & & Protein IGHV3-13 (Fragment) OS=Homo
\end{tabular}

$8 \quad 171 \quad \begin{aligned} & \text { Protein } I G H V 3-13 \text { (Fragment) } \\ & \text { sapiens } G N=I G H V 3-13 P E=1\end{aligned}$

8
9 172 Complement component 9, isoform CRA_a

\begin{tabular}{r|l|l}
9 & 172 & OS=Homo sapiens $\mathrm{GN}=\mathrm{C} 9 \mathrm{PE}=4 \mathrm{SV}=1$ \\
10 & & Protein disulfide-isomerase OS=Homo
\end{tabular}

$11 \quad 173$ sapiens $G N=P 4 H B \quad P E=3$ SV=1

$\begin{array}{llllllllll}\text { CXCL7_HUMAN (+1) } & 14 & 0.190 & 0.000 & 0.000 & 0.000 & 0.063 & 0.168 & 0.070 & 0.088\end{array}$ $\begin{array}{lllllllllll}\text { A0A024R930_HUMAN (+2) } & 151 & 0.042 & 0.033 & 0.035 & 0.054 & 0.111 & 0.132 & 0.068 & 0.043\end{array}$ $\begin{array}{llllllllll}\text { A0A0A0MS11_HUMAN } & 13 & 0.000 & 0.000 & 0.000 & 0.000 & 0.408 & 0.000 & 0.068 & 0.167\end{array}$ $\begin{array}{llllllllll}\text { A0A024R035_HUMAN (+1) } & 63 & 0.059 & 0.152 & 0.151 & 0.022 & 0.000 & 0.019 & 0.067 & 0.068\end{array}$ Epididymis luminal protein 180 (Fragment) OS=Homo sapiens $\mathrm{GN}=\mathrm{HEL} 180 \mathrm{PE}=2$ $174 \quad \mathrm{SV}=1$ Heat shock $70 \mathrm{kDa}$ protein $1 \mathrm{~B}$ OS=Homo

15 sapiens $\mathrm{GN}=\mathrm{HSPA} 1 \mathrm{~B} P \mathrm{PE}=1 \mathrm{SV}=1$ Alpha-1-acid glycoprotein 1 OS=Homo

176 sapiens $\mathrm{GN}=\mathrm{ORM} 1 \mathrm{PE}=1 \mathrm{SV}=1$ Zyxin OS=Homo sapiens GN=ZYX PE=1 $177 \quad$ SV=1

Anti-streptococcal/anti-myosin

immunoglobulin kappa light chain variable region (Fragment) OS=Homo sapiens

178 $\mathrm{PE}=2 \mathrm{SV}=1$

Catalase OS=Homo sapiens $\mathrm{GN}=\mathrm{CAT}$

$179 \quad \mathrm{PE}=1 \mathrm{SV}=3$ Integrin-linked protein kinase OS=Homo

180 sapiens $\mathrm{GN}=$ =ILK PE=1 SV=1

mo

Q96SA9_HUMAN

$12 \quad 0.000$ 0.000 0.159

$\begin{array}{lll}60 & 0.098 & 0.038\end{array}$
0.095

0.000

CATA_HUMAN

GCT-A5 light chain variable region

181 (Fragment) OS=Homo sapiens PE=2 SV=1 AOAOX9UWL5_HUMAN Myosin-reactive immunoglobulin heavy

chain variable region (Fragment)

$O S=H o m o$ sapiens $P E=2 S V=1$

Prothrombin (EC 3.4.21.5) OS=Homo

183 sapiens $P E=2$ SV=1

Prenylcysteine oxidase $10 \mathrm{OS}=\mathrm{Homo}$

184 sapiens $\mathrm{GN}=\mathrm{PCYOX} 1 \mathrm{PE}=1 \mathrm{SV}=3$

Carboxypeptidase $\mathrm{N}$ subunit $2 \mathrm{OS}=\mathrm{Homo}$

185

sapiens $\mathrm{GN}=\mathrm{CPN} 2 \mathrm{PE}=1 \mathrm{SV}=3$

V2-17 protein (Fragment) OS=Homo

186 sapiens $\mathrm{GN}=\mathrm{V} 2-17 \mathrm{PE}=1 \mathrm{SV}=1$

Multimerin-1 OS=Homo sapiens

187 GN=MMRN1 PE=1 SV=3

Ig gamma- 1 chain $C$ region OS=Homo

188 sapiens $\mathrm{GN}=\mid \mathrm{GHG} 1 \mathrm{PE}=1 \mathrm{SV}=1$

Tropomyosin 3 isoform 1 (Fragment)

189 OS=Homo sapiens GN=TPM3 PE=2 SV=1 Alpha-2-antiplasmin OS=Homo sapiens

190

$\mathrm{GN}=\mathrm{SERPINF} 2 \mathrm{PE}=1 \mathrm{SV}=3$

Calpain 1, (Mu/l) large subunit, isoform

CRA_a OS=Homo sapiens $\mathrm{GN}=\mathrm{CAPN} 1$

$191 \quad P E=4 \quad S V=1$

Ig heavy chain variable region (Fragment)

192 OS=Homo sapiens $P E=2 \mathrm{SV}=1$

Enolase 1, (Alpha), isoform CRA_a

193 OS=Homo sapiens GN=ENO1 PE=2 SV=1 Apolipoprotein A-IV OS=Homo sapiens

194 GN=APOA4 PE=1 SV=3

MS-D1 light chain variable region

195 (Fragment) OS=Homo sapiens PE=2 SV=1 Serpin peptidase inhibitor, clade $C$

(Antithrombin), member 1, isoform CRA_a OS=Homo sapiens GN=SERPINC1 PE=3

196

$\mathrm{SV}=1$

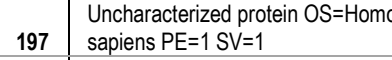

198 GCT-A8 heavy chain variable region

198 (Fragment) OS=Homo sapiens $\mathrm{PE}=2 \mathrm{SV}=$ Protein AMBP OS=Homo sapiens

199 GN=AMBP PE=1 SV=1

Fructose-bisphosphate aldolase $A$

$\mathrm{OS}=$ Homo sapiens $\mathrm{GN}=\mathrm{ALDOA} \mathrm{PE}=1$

$200 \quad S V=2$

Clathrin heavy chain OS=Homo sapiens

$201 \mathrm{GN}=\mathrm{CLTC} P E=1 \mathrm{SV}=1$

Inter-alpha-trypsin inhibitor heavy chain $\mathrm{H} 3$

202 OS=Homo sapiens GN=ITIH3 PE=1 SV=2

AOAOAOMTH3_HUMAN (+2)

$\begin{array}{lll}55 & 0.126 & 0.108\end{array}$

Q9UL89_HUMAN

$12 \quad 0.000$

0.000

0.000

0.000

$13 \quad 0.082$

0.000

0.293

$53 \quad 0.060$ 0.172

$\begin{array}{lll}57 & 0.019 & 0.056\end{array}$

PCYOX_HUMAN

CPN2_HUMAN

$\begin{array}{lll}61 & 0.070 & 0.097\end{array}$

$0.097 \quad 0.062$

$\begin{array}{lll}0.072 & 0.034 & 0.000\end{array}$

$0.096 \quad 0.078$

Q5NV90_HUMAN

MMRN1_HUMAN

$\begin{array}{lll}10 & 0.000 & 0.183\end{array}$

0.095

$0.067 \quad 0.036$

A0A087X1C7_HUMAN

$\begin{array}{lll}50 & 0.000 & 0.000\end{array}$

0.000

A0A0S2Z4G4_HUMAN (+1)

A2AP_HUMAN

$\begin{array}{lll}29 & 0.129 & 0.063\end{array}$

0.000

0.03

A2AP, HUMAN

A0A024R580_HUMAN (+2)

55

A0A068LRW6_HUMAN (+2)

$\begin{array}{lll}82 & 0.071 & 0.045\end{array}$

0.012

A0A024R4F1_HUMAN (+1)

APOA4_HUMAN

$\begin{array}{lll}14 & 0.114 & 0.000\end{array}$

0.102

$\begin{array}{lll}47 & 0.091 & 0.058\end{array}$

$\begin{array}{lll}45 & 0.000 & 0.041\end{array}$

0.051

A0A0X9TD47_HUMAN

$12 \quad 0.000$

0.000

0.000

0.000

0.000

$\quad 0.354$

0.000

0.03

$0.033 \quad 0.032$

$0.032 \quad 0.02$

0.162

.021

0.05

0.072

$0.058 \quad 0.037$

A0A024R944_HUMAN (+1)

$53 \quad 0.030$

0.129

0.099

$\begin{array}{lll}141 & 0.038 & 0.123\end{array}$

0.142

0.043

$\begin{array}{lll}13 & 0.000 & 0.000\end{array}$

A0A0X9V9C4_HUMAN

AMBP_HUMAN

$39 \quad 0.055$

0.140

0.330

0.061

0.03

0.02

022

0.165

$0.058 \quad 0.056$

ALDOA_HUMAN (+2)

$\begin{array}{lll}39 & 0.082 & 0.047\end{array}$

0.000

0.058

A0A087WVQ6_HUMAN (+1)

$\begin{array}{lll}192 & 0.103 & 0.031\end{array}$

ITIH3_HUMAN
100
0.059
0.100

0.015

$0.021 \quad 0.012$

0.076

0.02

0.01 
4 Pleckstrin OS=Homo sapiens GN=PLEK

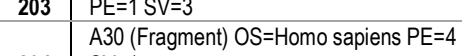

PLEK_HUMAN

$\begin{array}{lll}40 & 0.080 & 0.091\end{array}$

0.000

$\begin{array}{lll}0.000 & 0.000 \quad 0.103\end{array}$

$0.046 \quad 0.051$

Glycogen phosphorylase, brain form

A2MYE1_HUMAN (+1)

$\begin{array}{lll}10 & 0.000 & 0.000\end{array}$

0.000

$0.273 \quad 0.000$

0.000

$0.046 \quad 0.112$

$\mathrm{OS}=$ Homo sapiens $\mathrm{GN}=\mathrm{PYGB} P E=1 \mathrm{SV}=5$
Coagulation factor XIII B chain OS=Homo

sapiens $\mathrm{GN}=\mathrm{F} 13 \mathrm{~B} P \mathrm{PE}=1 \mathrm{SV}=3$

Rheumatoid factor RF-ET10 (Fragment)

207 OS=Homo sapiens $P E=2$ SV=1

cDNA FLJ61290, highly similar to Neutral

alpha-glucosidase $A B$ OS=Homo sapiens

208 PE=2 SV=1

$\begin{array}{lllllllllll}\text { PYGB_HUMAN (+1) } & 97 & 0.071 & 0.061 & 0.015 & 0.038 & 0.000 & 0.085 & 0.045 & 0.033\end{array}$

F13B HUMAN

$\begin{array}{lll}76 & 0.056 \quad 0.054\end{array}$

0.063

$0.048 \quad 0.017$

0.031

$0.045 \quad 0.017$

A2J1N7_HUMAN

$\begin{array}{lll}10 & 0.000 & 0.000\end{array}$

0.000

0.000

0.265

0.000

$0.044 \quad 0.108$

B4DJ30_HUMAN (+2)

$\begin{array}{lll}113 & 0.052 & 0.044\end{array}$

0.013

$0.016 \quad 0.008$

0.120

$0.042 \quad 0.042$

2 Phosphoglucomutase-1 OS=Hom

Complement component $1, q$

PGM1_HUMAN

$\begin{array}{lll}61 & 0.061 & 0.052\end{array}$

$\begin{array}{lll}0.000 & 0.022 & 0.000\end{array}$

0.116

subcomponent, A chain, isoform CRA

210 OS=Homo sapiens $G N=C 1 Q A P E=4$ SV $=1$ HCG2039812, isoform CRA_b (Fragment) $\mathrm{OS}=$ Homo sapiens $\mathrm{GN}=\mathrm{KRT} 6 \mathrm{~A} \mathrm{PE}=2$

$211 \quad S V=1$

A0A024RAG6_HUMAN (+2)

$\begin{array}{lll}26 & 0.061 & 0.035\end{array}$ 0.000

$0.035 \quad 0.051$

0.068

$0.042 \quad 0.024$

Protein IGKV2D-24 (Fragment) OS=Homo

sapiens $\mathrm{GN}=\mid \mathrm{GKV} 2 \mathrm{D}-24 \mathrm{PE}=4 \mathrm{SV}=1$

Protein IGHV1-46 (Fragment) OS=Homo

213 sapiens $\mathrm{GN}=I \mathrm{GHV} 1-46 \mathrm{PE}=4 \mathrm{SV}=1$

Class IVb beta tubulin OS=Homo sapiens

22

23

cDNA FLJ78516 OS=Homo sapiens $P E=2$

cDNA FLJ
SV $=1$

Transketolase (Fragment) OS=Homo

A0A0S2Z428_HUMAN (+3)

$\begin{array}{lllllllll}60 & 0.000 & 0.084 & 0.000 & 0.099 & 0.066 & 0.000 & 0.041 & 0.047\end{array}$

A0A075B6R9_HUMAN (+1)

$13 \quad 0.000$

0.000

0.000

0.105

0.000

0.136

$0.040 \quad 0.063$

A0A0B4J1V4_HUMAN (+1)

$\begin{array}{lll}13 & 0.000 & 0.070\end{array}$

0.000

$0.000 \quad 0.170$

Q8IWP6 HUMAN (+3)

\begin{tabular}{lllllllll}
50 & 0.000 & 0.000 & 0.000 & 0.000 & 0.000 & 0.235 & 0.039 & 0.096 \\
\hline
\end{tabular}

A8K2W3_HUMAN (+1)

$\begin{array}{lll}47 & 0.102 & 0.000\end{array}$

0.041

$0.029 \quad 0.000 \quad 0.063$

$0.039 \quad 0.039$

6 sapiens $P E=2 S V=1$

Myosin light polypeptide 6 OS=Homo

217 sapiens $\mathrm{GN}=\mathrm{MYL} 6 \mathrm{PE}=1 \mathrm{SV}=1$

Spectrin alpha chain, erythrocytic 1

$\mathrm{OS}=$ Homo sapiens $\mathrm{GN}=\mathrm{SPTA} 1 \mathrm{PE}=1$

218 SV=1

L-lactate dehydrogenase $B$ chain

Q53EM5_HUMAN (+2)

$\begin{array}{lll}68 & 0.063 & 0.040\end{array}$

0.000

B7Z6Z4_HUMAN (+6)

$\begin{array}{lll}27 & 0.138 & 0.000\end{array}$

0.000

$0.020 \quad 0.000$

0.104

$0.038 \quad 0.040$

AOA087WZE4 HUMAN $(+$

$281 \quad 0.072$

0.000

0.068

$37 \quad 0.043$

0.037

0.000

$0.000 \quad 0.000$

0.087

$0.038 \quad 0.060$

Mannan-binding lectin serine protease 1

$\mathrm{OS}=$ Homo sapiens $\mathrm{GN}=\mathrm{MASP} 1 \mathrm{PE}=1$

$220 \quad S V=3$

Complement component $\mathrm{C} 6$ OS=Homo

221 sapiens $\mathrm{GN}=\mathrm{C} 6 \mathrm{PE}=1 \mathrm{SV}=3$

GDP dissociation inhibitor 1 isoform 1

(Fragment) OS=Homo sapiens $\mathrm{GN}=\mathrm{GDI} 1$

$222 \quad \mathrm{PE}=2 \mathrm{SV}=1$

Ankyrin-1 OS=Homo sapiens GN=ANK1

$23 \quad P E=1 S V=3$

Ig heavy chain variable region (Fragment)

24 OS=Homo sapiens $\mathrm{PE}=2 \mathrm{SV}=1$

Cofilin-1 OS=Homo sapiens $\mathrm{GN}=\mathrm{CFL} 1$

$225 \quad P E=1 S V=3$

Polymeric immunoglobulin receptor

226 OS=Homo sapiens GN=PIGR PE=1 SV=4

Coronin OS=Homo sapiens $\mathrm{GN}=\mathrm{CORO} 1 \mathrm{~A}$

$227 \quad \mathrm{PE}=3 \mathrm{SV}=1$

Transferrin receptor protein $10 S=$ Homo

228 sapiens $G N=T F R C$ PE=1 $S V=2$

Hexokinase (Fragment) OS=Homo sapiens

$9 \mathrm{PE}=2 \mathrm{SV}=1$

Soluble scavenger receptor cysteine-rich

domain-containing protein SSC5D

$O S=H o m o$ sapiens $G N=S S C 5 D$ PE $=1$

MASP1_HUMAN

$\begin{array}{lll}79 & 0.067 & 0.040\end{array}$

0.018

$0.039 \quad 0.033$

0.033

0.013 $0.037 \quad 0.029$

CO6_HUMAN

$\begin{array}{lll}105 & 0.015 & 0.091\end{array}$
0.073

0.062

0.02

$0.000 \quad 0.079$
$0.037 \quad 0.032$

A0A0S2Z3X8_HUMAN (+2)

$\begin{array}{lll}51 & 0.104 & 0.027\end{array}$

0.019

0.013

0.028

0.030 $0.035 \quad 0.017$

ANK1_HUMAN

$\begin{array}{lll}206 & 0.067 & 0.000\end{array}$

0.067

$0.000 \quad 0.000$

0.058 $0.035 \quad 0.040$

A0A068LKQ2_HUMAN

$\begin{array}{lll}13 & 0.000 \quad 0.000\end{array}$

0.000

$0.031 \quad 0.021$

0.020

$0.034 \quad 0.027$

COF1_HUMAN $(+3)$

$\begin{array}{lllllllll}19 & 0.056 & 0.048 & 0.000 & 0.000 & 0.000 & 0.093 & 0.033 & 0.039\end{array}$

PIGR_HUMAN

$\begin{array}{lll}83 & 0.032 & 0.016\end{array}$

0.017

$\begin{array}{lll}0.022 & 0.107 & 0.000\end{array}$ $0.032 \quad 0.038$

A0A024R611_HUMAN (+1)

$51 \quad 0.084$

TFR1_HUMAN

$85 \quad 0.06$

Q59FD4_HUMAN

$\begin{array}{lll}106 & 0.040 \quad 0.034\end{array}$

0.027

$\begin{array}{lll}0.019 & 0.018 & 0.000\end{array}$

0.046

$0.032 \quad 0.029$

$S V=3$

CDNA FLJ55606, highly similar to Alpha-2-

HS-glycoprotein OS=Homo sapiens $\mathrm{PE}=2$

231 SV=1

Ig heavy chain V-III region JON OS=Homo

232 sapiens $P E=1 \mathrm{SV}=1$

Peptidyl-prolyl cis-trans isomerase

233 OS=Homo sapiens $P E=2$ SV=1

Spectrin, beta, erythrocytic (Includes

spherocytosis, clinical type I) variant

234 (Fragment) OS=Homo sapiens PE=2 SV= RAP1B, member of RAS oncogene family, isoform CRA_a OS=Homo sapiens

$235 \mathrm{GN}=\mathrm{RAP} 1 \mathrm{~B} P \mathrm{PE}=4 \mathrm{SV}=1$

236 Coagulation factor V OS=Homo sapiens

SRCRL_HUMAN

166 0.055 0.025 0.029 0.02

B7Z8Q2 HUMAN (+2)

$47 \quad 0.000$

0.078 0.091 0.019

HV319_HUMAN

$\begin{array}{lll}13 & 0.082 & 0.000\end{array}$

0.000 0.000

$18 \quad 0.000$ 0.000 0.000 0.051

Q59FP5_HUMAN 268

A0A024RB87_HUMAN (+3) $\begin{array}{rll}21 & 0.000 & 0.065\end{array}$ AOAOAOMRJ7_HUMAN (+1)

252 0.051 0.025 0.013 0.025 $0.000 \quad 0.000$ $\begin{array}{ll}0.021 & 0.112 \\ 0.042\end{array}$ $0.023 \quad 0.021$ 
$4 \quad$ GN=F5 PE=1 SV=1

5 Ubiquitin-activating enzyme E1 (A1S9T

and BN75 temperature sensitivity

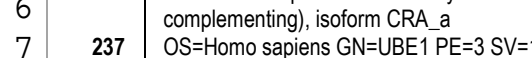

\begin{tabular}{l|l|l}
7 & 237 & CDNA, FLJ93914, highly similar to Homo
\end{tabular} sapiens histidine-rich glycoprotein (HRG),

9238 mRNA OS=Homo sapiens $P E=2 \quad S V=1$

Protein unc-13 homolog D OS=Homo

239 sapiens $G N=U N C 13 D P E=1 \quad S V=1$

Apolipoprotein A-II OS=Homo sapien

$240 \mathrm{GN}=\mathrm{APOA} 2 \mathrm{PE}=1 \mathrm{SV}=1$

Nucleosome assembly protein 1-like 1 ,

isoform CRA_a OS=Homo sapiens

$241 \mathrm{GN}=\mathrm{NAP} 1 \mathrm{~L} 1 \mathrm{PE}=3 \mathrm{SV}=1$

Serum paraoxonase/arylesterase 1

242 OS=Homo sapiens GN=PON1 PE=1 SV=

Protein IGKV2D-28 OS=Homo sapiens

$243 \mathrm{GN}=I G K V 2 D-28 \mathrm{PE}=1 \mathrm{SV}=1$

Beta-2-glycoprotein 1 OS=Homo sapiens

$244 \mathrm{GN}=\mathrm{APOH} P E=1 \mathrm{SV}=3$

CDNA FLJ50805, highly similar to

Erythrocyte membrane protein band 4.2

245 OS=Homo sapiens $P E=2 S V=1$

Transgelin-2 OS=Homo sapiens

$\mathrm{GN}=$ TAGLN2 PE=1 SV=3

Cryocrystalglobulin CC1 kappa light chain

variable region (Fragment) OS=Homo

247 sapiens $P E=2 S V=1$

Syntaxin-binding protein $2 \mathrm{OS}=\mathrm{Homo}$

248 sapiens $\mathrm{GN}=\mathrm{STXBP} 2 \mathrm{PE}=1 \mathrm{SV}=2$

Phosphoglycerate kinase OS=Homo

249 sapiens $P E=2 \mathrm{SV}=1$

cDNA FLJ77947, highly similar to Human

complement protein $\mathrm{C} 8$ beta subunit

250 mRNA OS=Homo sapiens $\mathrm{PE}=2 \mathrm{SV}=1$

Beta-parvin OS=Homo sapiens

251 GN=PARVB PE=1 SV=1

Programmed cell death 6 -interacting

protein OS=Homo sapiens GN=PDCD6IP

$252 \quad P E=1 S V=1$

T-complex protein 1 subunit gamma

253 OS=Homo sapiens PE=2 SV=1

Nicotinate phosphoribosyltransferase

(Fragment) OS=Homo sapiens

254 GN=NAPRT $P E=1 \mathrm{SV}=1$

A0A024R1A3_HUMAN (+1)

$\begin{array}{lll}118 & 0.050 & 0.043\end{array}$

0.012

0.000

0.000

0.070 $0.029 \quad 0.029$

B2R8I2_HUMAN (+1)

$60 \quad 0.036$

0.061

0.040

$0.023 \quad 0.015$

0.000

$0.029 \quad 0.021$

UN13D_HUMAN

$\begin{array}{lll}123 & 0.052 & 0.026\end{array}$

0.000

0.00

APOA2_HUMAN (+3)

$\begin{array}{lll}11 & 0.000 & 0.000\end{array}$

0.000

0.16

A0A024RBB7_HUMAN (+12)

$\begin{array}{lll}45 & 0.059 & 0.030\end{array}$

0.021

$0.000 \quad 0.000$

$\begin{array}{lll}40 & 0.000 & 0.068\end{array}$

0.048

$0.046 \quad 0.000$

0.000

0.025

AOAOAOMTQ6

$\begin{array}{lll}11 & 0.000 & 0.000\end{array}$

APOH_HUMAN (+1)

$38 \quad 0.000$

0.060

0.000

$0.000 \quad 0.161$

0.000

$0.027 \quad 0.031$

$G N=N A P R T P E=1 S V=1$

Glucose-6-phosphate isomerase
(Fragment) OS=Homo sapiens $\mathrm{GN}=\mathrm{GP}$

$255 \quad P E=1 S V=1$

Arachidonate 12-lipoxygenase, 12S-type

OS=Homo sapiens GN=ALOX12 PE=1

256 SV $=4$

ATP-dependent 6-phosphofructokinase,

platelet type OS=Homo sapiens GN=PFKP

$257 \quad P E=1 \mathrm{SV}=2$

IQ motif containing GTPase activating

protein 2, isoform CRA_b OS=Homo

258 sapiens $\mathrm{GN}=\mid \mathrm{QGAP} 2 \mathrm{PE}=4 \mathrm{SV}=1$

Glucose-6-phosphate 1-dehydrogenase

259 OS=Homo sapiens $\mathrm{GN}=\mathrm{G} 6 \mathrm{PD} P \mathrm{PE}=1 \mathrm{SV}=4$ Nidogen-1 OS=Homo sapiens GN=NID1

$260 \quad P E=1 S V=3$

cDNA, FLJ95650, highly similar to Homo

sapiens karyopherin (importin) beta 1

(KPNB1), mRNA OS=Homo sapiens $\mathrm{PE}=2$

$261 \quad S V=1$

SV $=1$

complement protein component $\mathrm{C} 7 \mathrm{mRNA}$

262 OS=Homo sapiens PE=2 SV=1

Chloride intracellular channel protein 1

54

55

56

$77 \quad 0.055$

TAGL2_HUMAN (+1)

$\begin{array}{lll}22 & 0.048 & 0.000\end{array}$

0.100

0.000

$0.000 \quad 0.000$

$\begin{array}{llll}0.000 & 0.000 & 0.027 & 0.043\end{array}$

B1N7B8_HUMAN

$\begin{array}{lll}12 & 0.000 \quad 0.000\end{array}$

$0.035 \quad 0.017$

0.017

0.000

$0.026 \quad 0.024$

STXB2_HUMAN

B4E1H9_HUMAN (+2)

$\begin{array}{lll}66 & 0.032 & 0.035\end{array}$

0.000

$\begin{array}{lll}0.000 & 0.000 & 0.107\end{array}$

$0.026 \quad 0.044$

A8K9M5 HUMAN (+6)

$\begin{array}{lll}35 & 0.030 & 0.039\end{array}$

$\begin{array}{lll}0.000 & 0.152 & 0.000\end{array}$

0.000

$0.025 \quad 0.062$

PARVB_HUMAN

$\begin{array}{lll}67 & 0.024 & 0.048\end{array}$

0.000

$0.000 \quad 0.000$

0.080

$0.024 \quad 0.032$

PDC6I_HUMAN $(+1)$

$\begin{array}{lll}42 & 0.038 & 0.033\end{array}$

0.071

0.000

$026 \quad 0.000$

$0.000 \quad 0.050$

$\begin{array}{lll}0.050 & 0.024 & 0.021\end{array}$

PDC6I_HUMAN (+1)

$\begin{array}{lll}96 & 0.028 & 0.019\end{array}$

0.000

0.000

0.000

$0.024 \quad 0.030$

B3KX11_HUMAN (+4)

$\begin{array}{lll}58 & 0.064 & 0.000\end{array}$

0.000

$0.000 \quad 0.000$

0.070

$0.023 \quad 0.029$

HOYDA6 HUMAN

HOYDA6_HUMAN

$14 \quad 0.000-0.000$

$0.000 \quad 0.063$

AOAOAOMTS2 HUMAN

$65 \quad 0.066$

0.042

0.022

$0.000 \quad 0.000$

0.000

$0.022 \quad 0.027$

LOX12 HUMAN

$\begin{array}{lll}76 & 0.056 & 0.000\end{array}$

0.025

$0.000 \quad 0.000$

0.046

$0.021 \quad 0.025$

PFKAP HUMAN

$\begin{array}{lll}86 & 0.037 & 0.053\end{array}$

0.000

$0.000 \quad 0.000$

0.034

$0.021 \quad 0.024$

A0A024RAJ8_HUMAN (+2)

$\begin{array}{lll}181 & 0.032 & 0.023\end{array}$

0.000

$0.013 \quad 0.000$

0.055

$0.020 \quad 0.021$

G6PD_HUMAN

$\begin{array}{lll}59 & 0.054 & 0.031\end{array}$

0.016

$0.000 \quad 0.000$

0.020

$0.020 \quad 0.021$

NID1_HUMAN

$\begin{array}{lll}136 & 0.051 \quad 0.007\end{array}$ 0.000 0.013

B2RBR9_HUMAN (+1)

$\begin{array}{lll}97 & 0.044 & 0.024\end{array}$

0.010

$0.000 \quad 0.000$

0.042

$0.020 \quad 0.020$

A8K2T4 HUMAN (+2)

$\begin{array}{lll}93 & 0.000 & 0.074\end{array}$

0.046

$0.000 \quad 0.000$

0.000 $0.020 \quad 0.032$

OS=Homo sapiens $\mathrm{GN}=\mathrm{CLIC} 1 \mathrm{PE}=1 \mathrm{SV}=$

Plasma kallikrein (Fragment) OS=Homo

264 sapiens $\mathrm{GN}=\mathrm{KLKB} 1 \mathrm{PE}=1 \mathrm{SV}=1$

Rho-associated protein kinase 2

$\mathrm{OS}=$ Homo sapiens $\mathrm{GN}=\mathrm{ROCK} 2 \mathrm{PE}=1$

$265 \quad S V=4$

Tyrosine-protein phosphatase non-

\begin{tabular}{ll|llll}
60 & 266 & PTN6_HUMAN $(+2)$ & 68 & 0.024 & 0.034 \\
61 & & & & 0.000 \\
62 & & & & & \\
63 & & & & & \\
64 & & &
\end{tabular}

CLIC1_HUMAN (+2)

HOYAC1_HUMAN (+1)

$\begin{array}{lll}27 & 0.039 & 0.034\end{array}$

0.000

$0.000 \quad 0.000$

0.044

$0.019 \quad 0.022$

$\begin{array}{lll}77 & 0.014 & 0.041\end{array}$

0.049

0.012

ROCK2_HUMAN

$\begin{array}{lll}161 & 0.033 & 0.014\end{array}$

0.000

$0.000 \quad 0.000$

0.066

$0.019 \quad 0.026$

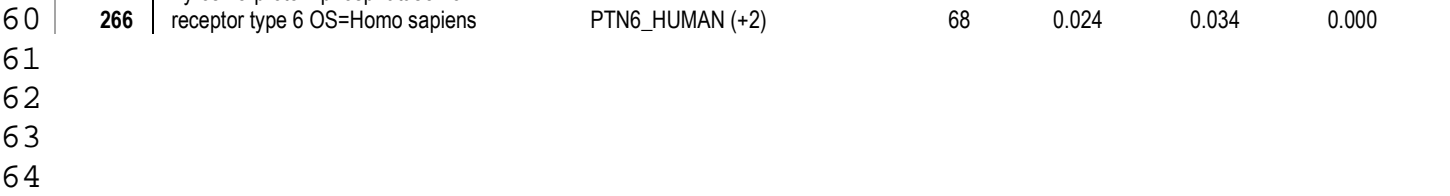

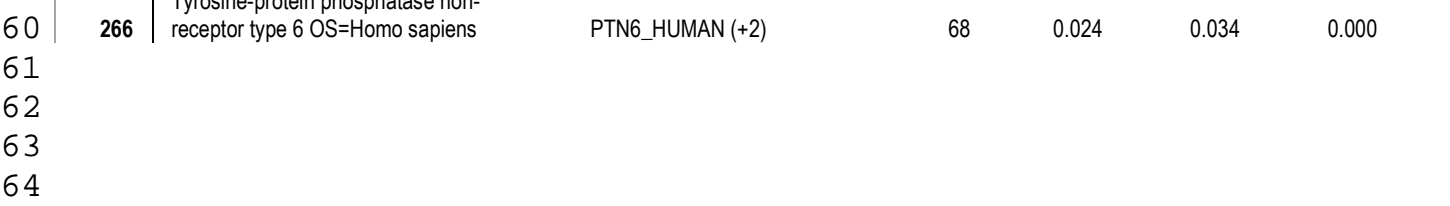

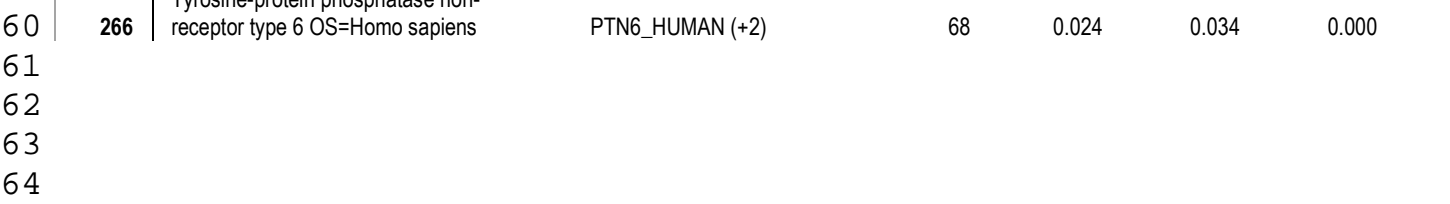

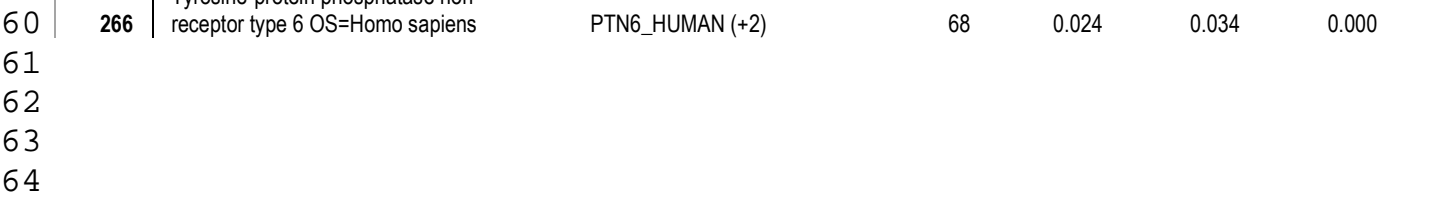

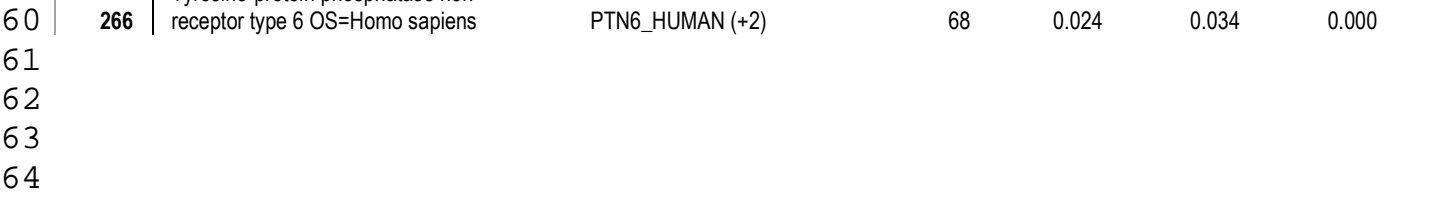

0.02 


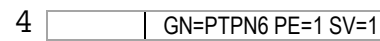

267 sapiens $P E=2 \mathrm{SV}=1$

CDNA FLJ50442, highly similar to T-

B4DUI5_HUMAN (+4)

$23 \quad 0.069 \quad 0.040$ 0.000 $0.000 \quad 0.000$ 0.000 $0.018 \quad 0.030$

complex protein 1 subunit epsilon

8268 OS=Homo sapiens $P E=2 \mathrm{SV}=1$

9269 Ig heavy chain variable region (Fragment)

$\mathrm{OS}=$ Homo sapiens $\mathrm{PE}=2 \mathrm{SV}=1$

Protein IGKV1-16 (Fragment) OS=Homo

270 sapiens $G N=\mid G K V 1-16 \quad P E=1 \mathrm{SV}=1$

Chaperonin containing TCP1, subunit 7

(Eta) variant (Fragment) OS=Homo

\begin{tabular}{lccccccccc} 
B4DDU6_HUMAN (+5) & 55 & 0.048 & 0.017 & 0.000 & 0.000 & 0.000 & 0.043 & 0.018 & 0.022 \\
A0A068LN03_HUMAN & 13 & 0.000 & 0.000 & 0.000 & 0.105 & 0.000 & 0.000 & 0.018 & 0.043 \\
\hline A0A0A0MT74_HUMAN & 13 & 0.000 & 0.000 & 0.000 & 0.105 & 0.000 & 0.000 & 0.018 & 0.043 \\
Q53HV2_HUMAN (+1) & 59 & 0.036 & 0.039 & 0.000 & 0.000 & 0.000 & 0.030 & 0.017 & 0.019 \\
\hline CO8A_HUMAN & 65 & 0.025 & 0.042 & 0.037 & 0.000 & 0.000 & 0.000 & 0.017 & 0.020 \\
\hline A0A024RBI5_HUMAN (+3) & 53 & 0.080 & 0.000 & 0.000 & 0.000 & 0.000 & 0.022 & 0.017 & 0.032 \\
\hline A0A0C4DH83_HUMAN (+1) & 62 & 0.026 & 0.022 & 0.000 & 0.015 & 0.000 & 0.038 & 0.017 \\
\hline
\end{tabular}

275 SV=2 Chaperonin containing TCP1, subunit 8

(Theta) variant (Fragment) OS=Homo

276 sapiens $P E=2 ~ S V=1$

Protein disulfide-isomerase A6 OS=Homo

Complement component $\mathrm{C} 8$ alpha chain

2 OS=Homo sapiens $\mathrm{GN}=\mathrm{C} 8 \mathrm{~A} \mathrm{PE}=1 \mathrm{SV}=2$

Coronin OS=Homo sapiens $\mathrm{GN}=\mathrm{CORO} 1 \mathrm{C}$

$P E=3 S V=1$

EH domain-containing protein 3 OS=Homo

4 sapiens $\mathrm{GN}=\mathrm{EHD} 3 \mathrm{PE}=1 \mathrm{SV}=1$

sapiens $G N=P D I A 6 P E=1 S V=1$

Protein S100-A8 OS=Homo sapiens

$8 \mathrm{GN}=\mathrm{S} 100 \mathrm{~A} 8 \mathrm{PE}=1 \mathrm{SV}=1$

Capping protein (Actin filament) muscle Z-

line, beta, isoform CRA_a OS=Homo

279 sapiens $\mathrm{GN}=\mathrm{CAPZB} P E=1 \mathrm{SV}=1$

Rheumatoid factor RF-IP4 (Fragment)

280 OS=Homo sapiens $P E=2 \mathrm{SV}=1$

LTBP1 protein OS=Homo sapiens

281 GN=LTBP1 PE=2 SV=1

cDNA FLJ51409, highly similar to

Thrombospondin-4 OS=Homo sapiens

$282 \mathrm{PE}=2 \mathrm{SV}=1$

Alpha-1,4 glucan phosphorylase

283 OS=Homo sapiens $P E=2 S V=1$

Serum amyloid $\mathrm{P}$-component $\mathrm{OS}=\mathrm{Homo}$

284 sapiens $\mathrm{GN}=\mathrm{APCS} P E=1 \mathrm{SV}=2$

Cytoplasmic FMR1 interacting protein 1

isoform $A$ (Fragment) OS=Homo sapiens

$285 \mathrm{GN}=\mathrm{CYFIP} 1 \mathrm{PE}=2 \mathrm{SV}=1$

286 Protein disulfide-isomerase A4 OS=Homo

sapiens $\mathrm{GN}=\mathrm{ERP} 70 \mathrm{PE}=3 \mathrm{SV}=1$

cDNA FLJ50491, highly similar to Amyloid

beta A4 protein (APP) (ABPP)(Alzheimer

disease amyloid protein) (Cerebral

vascularamyloid peptide) (CVAP)

(Protease nexin-II) (PN-II)(APPI) (PreA4)

287 OS=Homo sapiens $P E=2$ SV=1

CD36 antigen (Collagen type I receptor,

thrombospondin receptor) OS=Homo

288 sapiens $\mathrm{GN}=\mathrm{CD} 36 \mathrm{PE}=2 \mathrm{SV}=1$

L-lactate dehydrogenase $A$ chain

-

$00-0.044-0.015$

Q53HUO_HUMAN (+2)

$\begin{array}{llll}60 & 0.044 & 0.015 & 0.000\end{array}$

$0.000 \quad 0.000$

PDIA6_HUMAN

$\begin{array}{lll}48 & 0.022 & 0.038\end{array}$

S10A8_HUMAN

$\begin{array}{lll}11 & 0.097 & 0.000\end{array}$

0.000

$0.000 \quad 0.000$

0.000

0.039

$0.016 \quad 0.021$

289 OS=Homo sapiens GN=LDHA PE=1

Proto-oncogene tyrosine-protein kinase

$\mathrm{Src}$ OS=Homo sapiens $\mathrm{GN}=\mathrm{SRC} \mathrm{PE}=1$

$290 \quad S V=3$

$\mathrm{V}$-type proton ATPase catalytic subunit A

OS=Homo sapiens GN=ATP6V1A PE=1

$291 \quad \mathrm{SV}=2$

Heat shock 60kDa protein 1 (Chaperonin),

isoform CRA_a OS=Homo sapiens

292 GN=HSPD1 $\mathrm{PE}=2 \mathrm{SV}=1$

cDNA, FLJ93545, highly similar to Homo

sapiens 5-aminoimidazole-4-carboxamide

ribonucleotide formyltransferase/IMP

cyclohydrolase (ATIC), mRNA OS=Homo

293 sapiens $P E=2$ SV=1

cDNA, FLJ92620, highly similar to Homo

sapiens staphylococcal nuclease domain

containing 1 (SND1), mRNA OS=Homo

294 sapiens $P E=2 S V=1$

295 Ubiquitin-like modifier-activating enzyme 7

B1AK87_HUMAN (+3)

$\begin{array}{lll}29 & 0.055 & 0.000\end{array}$

0.000

$\begin{array}{lll}0.000 & 0.000 & 0.000\end{array}$

\title{
A2J1M5_HUMAN
}

B7ZLY3_HUMAN (+3)

$\begin{array}{lll}10 & 0.000 & 0.000\end{array}$

0.000

$0.000 \quad 0.000$

0.041

$0.016 \quad 0.025$

B7Z832 HUMAN $(+2)$

$\begin{array}{lll}148 & 0.029 & 0.009\end{array}$

$\begin{array}{lll}0.095 & 0.000 & 0.000\end{array}$

0.000

0.000

$0.016 \quad 0.039$

B7Z832_HUMAN (+2)

B2R825_HUMAN (+1)

$96 \quad 0.028$

0.010

0.015

$0.009 \quad 0.014$

0.018

0.01

SAMP_HUMAN (+1)

$\begin{array}{lll}97 & 0.038 & 0.000\end{array}$

$\begin{array}{lll}0.000 & 0.000 & 0.000\end{array}$

0.055

$0.015 \quad 0.025$

-

X5D2F4_HUMAN

$\begin{array}{lll}25 & 0.000 & 0.055\end{array}$

0.038

0.000

A0A090N8Y2_HUMAN (+1)

$\begin{array}{lll}145 & 0.029 & 0.019\end{array}$

0.000

0.006

0.000

0.000

$0.015 \quad 0.025$

AOA090NBY2 HUMAN $(+1)$

$\begin{array}{ll}73 & 0.029\end{array}$

0.013

0.000 0.000

0.000

0.036

$0.015 \quad 0.016$

(2)

\author{
B4DGDO_HUMAN
}

$\begin{array}{lll}81 & 0.046 & 0.000\end{array}$

0.000

$0.000 \quad 0.000$

0.044

$0.015 \quad 0.023$

A4D1B1_HUMAN (+6)

$\begin{array}{lll}53 & 0.020 & 0.000\end{array}$

0.000

$0.000 \quad 0.000$

0.067

$0.014 \quad 0.027$

LDHA_HUMAN (+1)

$\begin{array}{lll}37 & 0.000 & 0.037\end{array}$

0.000

0.00

SRC_HUMAN

$\begin{array}{lll}60 & 0.027 & 0.038\end{array}$

0.000

VATA HUMAN

$\begin{array}{lll}68 & 0.024 & 0.000\end{array}$

0.000

A0A024R3X4_HUMAN (+2)

61

0.035

0.000

0.000

$0.000 \quad 0.000$

0.048

0.01

$0.014 \quad 0.022$

B2R7P8_HUMAN (+2)

65

0.041

0.014

0.000 100

UBA7_HUMAN

$100 \quad 0.027$

$\begin{array}{lll}100 & 0.027 & 0.014 \\ 112 & 0.010 & 0.024\end{array}$

\begin{tabular}{lllll|}
0.000 & 0.000 & 0.060 & 0.014 & 0.025 \\
0.000 & 0.000 & 0.048 & 0.014 & 0.022 \\
& & & & \\
& & & & \\
0.000 & 0.000 & 0.027 & 0.014 & 0.017 \\
& & & & \\
0.000 & 0.000 & 0.041 & 0.014 & 0.017 \\
\hline 0.000 & 0.000 & 0.047 & 0.014 & 0.019
\end{tabular}


$4 \quad$ OS=Homo sapiens GN=UBA7 PE=1 SV=2

Angiotensinogen variant (Fragment)

$\mathrm{OS}=$ Homo sapiens $\mathrm{PE}=2 \mathrm{SV}=1$

(Fragment) OS=Homo sapiens $\mathrm{PE}=2 \mathrm{SV}=1$ Alpha-1-acid glycoprotein $2 \mathrm{OS}=\mathrm{Homo}$

299 sapiens $\mathrm{GN}=\mathrm{ORM} 2 \mathrm{PE}=1 \mathrm{SV}=2$ Isocitrate dehydrogenase [NADP], mitochondrial OS=Homo sapiens

$300 \mathrm{GN}=\mathrm{IDH} 2 \mathrm{PE}=1 \mathrm{SV}=2$ Integrin beta-1 OS=Homo sapiens

$301 \mathrm{GN}=\mathrm{ITGB} 1 \mathrm{PE}=1 \mathrm{SV}=2$

Bridging integrator $2 \mathrm{OS}=\mathrm{Homo}$ sapiens

302 GN=BIN2 $P E=1 \mathrm{SV}=1$

Protein diaphanous homolog 1 OS=Homo

303 sapiens $\mathrm{GN}=\mathrm{DIAPH} 1 \mathrm{PE}=1 \mathrm{SV}=1$

Chaperonin containing TCP1, subunit $6 \mathrm{~A}$

(Zeta 1), isoform CRA_a OS=Homo

304 sapiens $\mathrm{GN}=\mathrm{CCT} 6 \mathrm{~A}$ PE $=3 \mathrm{SV}=1$ Lipopolysaccharide-binding protein

305 OS=Homo sapiens GN=LBP PE=1 SV=3

Oncoprotein-induced transcript 3 protein

306 OS=Homo sapiens $\mathrm{GN}=\mathrm{OIT} 3 \mathrm{PE}=1 \mathrm{SV}=2$ Calcium-transporting ATPase OS=Homo

307 sapiens $\mathrm{PE}=2 \mathrm{SV}=1$

Lumican OS=Homo sapiens GN=LUM

$P E=1 S V=2$

cDNA, FLJ94361, highly similar to Homo

sapiens serine (or cysteine) proteinase

inhibitor, clade A(alpha-1 antiproteinase,

antitrypsin), member 6 (SERPINA6),

309 mRNA OS=Homo sapiens $P E=2$ SV=1

Hydroxysteroid (17-beta) dehydrogenase

4, isoform CRA_b (Fragment) OS=Homo

310 sapiens $\mathrm{GN}=\mathrm{HSD} 17 \mathrm{~B} 4 \mathrm{PE}=2 \mathrm{SV}=1$

Anti-HER3 scFv (Fragment) OS=Homo

311 sapiens $\mathrm{PE}=2 \mathrm{SV}=1$

Ig heavy chain $V$-III region TRO OS=Homo

312 sapiens $\mathrm{PE}=1 \mathrm{SV}=1$

Beta-arrestin-1 (Fragment) OS=Homo

313 sapiens $\mathrm{GN}=\mathrm{ARRB} 1 \mathrm{PE}=1 \mathrm{SV}=7$

$\mathrm{N}$-acetylmuramoyl-L-alanine amidase

OS=Homo sapiens GN=PGLYRP2 PE=1

$314 \quad \mathrm{SV}=1$

Fibulin-1 OS=Homo sapiens GN=FBLN1

315

$P E=1 S V=4$

Selectin $\mathrm{P}$ (Granule membrane protein

$140 \mathrm{kDa}$, antigen CD62), isoform CRA_b

316 OS=Homo sapiens GN=SELP PE=4 SV=1

LIM and senescent cell antigen-like-

containing domain protein $3 \mathrm{OS}=\mathrm{Homo}$

317 sapiens $\mathrm{GN}=\mathrm{LIMS4} \mathrm{PE}=1 \mathrm{SV}=1$

T-complex protein 1 subunit delta

318 OS=Homo sapiens $\mathrm{PE}=2 \mathrm{SV}=1$

T-complex protein 1 subunit alpha

319 OS=Homo sapiens $\mathrm{GN}=\mathrm{TCP} 1 \mathrm{PE}=1 \mathrm{SV}=1$

Glutathione reductase, mitochondrial

320 OS=Homo sapiens $G N=G S R \quad P E=1 S V=2$

Phospholipase A2, group VII (Platelet-

activating factor acetylhydrolase, plasma),

isoform CRA_a OS=Homo sapiens

$321 \mathrm{GN}=\mathrm{PLA} 2 \mathrm{G} 7 \mathrm{PE}=4 \mathrm{SV}=1$

14-3-3 protein eta OS=Homo sapiens

$322 \mathrm{GN}=\mathrm{YWHAH} \mathrm{PE}=1 \mathrm{SV}=4$

Tripeptidyl-peptidase 2 OS=Homo sapiens

323 GN=TPP2 PE=1 SV=1

cGMP-dependent protein kinase 1

OS=Homo sapiens $\mathrm{GN}=\mathrm{PRKG} 1 \mathrm{PE}=1$

324 SV=1

Alanine--tRNA ligase, cytoplasmic

325 OS=Homo sapiens GN=AARS PE=1 SV=2

Complement factor I OS=Homo sapiens

$326 \mathrm{GN}=\mathrm{CFI} P E=1 \mathrm{SV}=2$

Reelin OS=Homo sapiens GN=RELN

$327 \quad \mathrm{PE}=1 \mathrm{SV}=1$

328 Dipeptidyl peptidase 3 OS=Homo sapiens

\section{AFAM_HUMAN}

Q53GY3_HUMAN (+3)

$\begin{array}{lll}69 & 0.000 & 0.040\end{array}$

0.028

$0.013 \quad 0.000$

0.000

$0.013 \quad 0.017$

Q59GB4_HUMAN

$\begin{array}{lll}53 & 0.000 & 0.017\end{array}$

0.063

$0.000 \quad 0.000$

$\begin{array}{lll}68 & 0.063 & 0.000\end{array}$

0.000

0.00

A1AG2_HUMAN

$\begin{array}{lll}24 & 0.000 & 0.000\end{array}$

0.079

0.000

IDHP_HUMAN (+1)

$\begin{array}{lll}51 & 0.021 & 0.000\end{array}$

ITB1_HUMAN

$\begin{array}{lll}88 & 0.018 & 0.000\end{array}$

$\begin{array}{lll}0.000 & 0.000 & 0.000\end{array}$

A0A087X188_HUMAN (+4)

$\begin{array}{lll}65 & 0.025 & 0.000\end{array}$

0.000

A0A0G2JH68_HUMAN (+3)

$141 \quad 0.019$

A0A024RDL1_HUMAN (+2)

LBP_HUMAN (+1)

$\begin{array}{lll}58 & 0.055 & 0.000\end{array}$

OIT3_HUMAN

A8K9K1_HUMAN (+1)

LUM_HUMAN (+1)

$\begin{array}{lll}53 & 0.000 & 0.017\end{array}$

$\begin{array}{lll}60 & 0.036 & 0.000\end{array}$

$\begin{array}{lll}109 & 0.015 & 0.000\end{array}$

38

0.000

0.036

B2R9F2_HUMAN (+1)

$\begin{array}{lll}45 & 0.000 & 0.051\end{array}$

0.021

$0.000 \quad 0.000$

0.000

A0A0S2Z4J1_HUMAN (+2)

$\begin{array}{lll}80 & 0.020 & 0.000\end{array}$

0.000

$0.000 \quad 0.000$

0.051

$0.012 \quad 0.021$

A2J422_HUMAN

$\begin{array}{lll}26 & 0.000 & 0.070\end{array}$

0.000

$0.000 \quad 0.000$

0.000

0.012

HV301_HUMAN

$\begin{array}{lll}13 & 0.000 & 0.000\end{array}$

0.000

$0.070 \quad 0.000$

E9PM35_HUMAN

$\begin{array}{lll}29 & 0.037 & 0.031\end{array}$

0.000

PGRP2_HUMAN

$\begin{array}{lll}62 & 0.000 & 0.037\end{array}$

FBLN1_HUMAN

$77 \quad 0.000$

0.041

0.031

91

A0A024R8Y9_HUMAN (+7)

$91 \quad 0.018$

0.015

0.000

$\begin{array}{lll}46 & 0.035 & 0.030\end{array}$

0.000

0.00

A8K3C3_HUMAN (+1)

$\begin{array}{lll}58 & 0.064 & 0.000\end{array}$

$60 \quad 0.044$

0.000

0.000

$\begin{array}{lll}56 & 0.048 & 0.016\end{array}$

GSHR_HUMAN (+1)

0.000

0.000

A0A024RD39_HUMAN (+2)

50

1433F_HUMAN (+1)

$0.000-0.000$

0.000

0.036

0.027

0.000

0.010

Q5VZU9_HUMAN (+1)

$140-0.038=0.007$

0.000

AOA0AOMSB3_HUMAN (+1)

SYAC_HUMAN

CFAI_HUMAN (+2)

J3KQ66_HUMAN (+1)

DPP3_HUMAN (+2)
$52 \quad 0.000 \quad 0.026$

$\begin{array}{lll}107 & 0.010 & 0.009\end{array}$

388

83

$\begin{array}{lll}66 & 0.016 & 0.014\end{array}$

0.000

$0.000 \quad 0.000$

0.000

$0.009 \quad 0.000$

0.033

$0.010 \quad 0.016$

0.029

$0.000 \quad 0.000$

0.000
0.01

\begin{tabular}{ll}
.011 & 0.018 \\
\hline .011 & 0.016 \\
\hline & \\
\hline .011 & 0.013 \\
\hline & \\
\hline 011 & 0.017 \\
\hline
\end{tabular}

$\begin{array}{lll}0.025 & 0.011 & 0.005\end{array}$

0.005

0.000

0.000

0.000

0.035

$\begin{array}{ll}0.010 & 0.010 \\ 0.009 & 0.015\end{array}$ 
$4 \quad$\begin{tabular}{l|l}
\hline & GN=DPP3 PE=1 SV=2
\end{tabular}

\begin{tabular}{l|l|l}
5 & $\begin{array}{l}\text { TNC variant protein (Fragment) } \mathrm{OS}=\text { Homo } \\
\text { sapiens } \mathrm{GN}=\mathrm{TNC} \text { variant protein } \mathrm{PE}=2\end{array}$
\end{tabular}

Phosphodiesterase $5 \mathrm{~A}$ OS=Homo sapien

$330 \quad \mathrm{PE}=2 \mathrm{SV}=1$

Carboxypeptidase $\mathrm{N}$ catalytic chain

IgG $\mathrm{H}$ chain $\mathrm{OS}=$ Homo sapiens $\mathrm{PE}=2$

SV $=1$

NAD-dependent malic enzyme,

mitochondrial OS=Homo sapiens $\mathrm{GN}=\mathrm{ME} 2$

cDNA FLJ56381, highly similar to

Dynamin-1-like protein (EC 3.6.5.5)

334 OS=Homo sapiens $\mathrm{PE}=2 \mathrm{SV}=1$

Thyroxine-binding globulin OS=Homo

335 sapiens $\mathrm{GN}=\mathrm{SERPINA7} \mathrm{PE=1} \mathrm{SV=2}$

Ras-related $\mathrm{C} 3$ botulinum toxin substrate 2

(Rho family, small GTP binding protein

Rac2), isoform CRA_a OS=Homo sapiens

$336 \quad \mathrm{GN}=\mathrm{RAC2} P E=3 \mathrm{SV}=1$

Collagen alpha-1(VI) chain OS=Homo

337 sapiens $\mathrm{GN}=\mathrm{COL} 6 \mathrm{~A} 1 \mathrm{PE}=1 \mathrm{SV}=1$

Rho GTPase activating protein OS=Homo

Q4LE33_HUMAN

244

$0.017 \quad 0.006$

0.000

$0.011 \quad 0.020$

0.000

$0.009 \quad 0.009$

$\begin{array}{llllllllll}\text { I6NLS4_HUMAN (+1) } & 100 & 0.000 & 0.018 & 0.000 & 0.000 & 0.000 & 0.035 & 0.009 & 0.015\end{array}$

\begin{tabular}{llllllllll} 
CBPN_HUMAN & 52 & 0.000 & 0.035 & 0.018 & 0.000 & 0.000 & 0.000 & 0.009 & 0.015 \\
\hline
\end{tabular}

S6AWFO_HUMAN

$25 \quad 0.000 \quad 0.000$

0.000

0.000

0.053

0.000

$0.009 \quad 0.022$

MAOM_HUMAN

$65 \quad 0.025$

0.000

0.000

$0.000 \quad 0.000$

0.027

$0.009 \quad 0.013$

B4DYR6_HUMAN (+5)

$\begin{array}{lll}85 & 0.013 & 0.011\end{array}$

0.000

$0.000 \quad 0.000$

0.028

$0.008 \quad 0.011$

THBG_HUMAN

$\begin{array}{lll}46 & 0.000 & 0.020\end{array}$

0.031

0.000

A0A024R1P2_HUMAN (+5)

$21 \quad 0.051 \quad 0.000$

0.000

$0.000 \quad 0.000$

0.000

0.00

A0A087X0S5_HUMAN (+1)

$\begin{array}{lll}108 & 0.020 & 0.008\end{array}$

0.000

$0.000 \quad 0.000$

A9UK01_HUMAN (+1)

$\begin{array}{lll}75 & 0.014 & 0.000\end{array}$

0.000

$\begin{array}{lll}0.012 & 0.000 & 0.024\end{array}$

0.00

ATP-citrate synthase OS=Homo sapiens

$39 \quad \mathrm{GN}=A C L Y \quad P E=3 \quad S V=1$

A0A024R1T9_HUMAN (+3)

$\begin{array}{lll}121 & 0.018 & 0.008\end{array}$

0.000

0.000

Proteoglycan 1 , secretory granule, isoform

$340 \quad \mathrm{PE}=4 \mathrm{SV}=1$

Coatomer subunit alpha OS=Homo

341 sapiens $\mathrm{GN}=\mathrm{COPA} \mathrm{PE}=1 \mathrm{SV}=2$

cDNA FLJ78437, highly similar to Homo

sapiens cartilage oligomeric matrix protein

(COMP), mRNA OS=Homo sapiens $\mathrm{PE}=2$

$342 \quad \mathrm{SV}=1$

Glutathione S-transferase P OS=Homo

343 sapiens $\mathrm{GN}=\mathrm{GSTP} 1 \mathrm{PE}=1 \mathrm{SV}=2$

IgG H chain OS=Homo sapiens $P E=2$

$344 \quad S V=1$

345 Fibulin-1 OS=Homo sapiens PE=2 SV=1

A0A024QZL1_HUMAN (+1)

$\begin{array}{lll}18 & 0.000 & 0.000\end{array}$

COPA_HUMAN

$\begin{array}{lll}138 & 0.027 & 0.000\end{array}$

0.000

0.000

0.000

0.02

$0.008 \quad 0.010$

Ras suppressor protein 1 variant 5

346 OS=Homo sapiens GN=RSU1 PE=4 SV=1 Integrin alpha-6 OS=Homo sapiens

$347 \quad \mathrm{GN}=I T G A 6 \mathrm{PE}=1 \mathrm{SV}=5$

PDZ and LIM domain protein 1 OS=Homo

348

sapiens $\mathrm{GN}=\mathrm{PDLIM} 1 \mathrm{PE}=1 \mathrm{SV}=4$

Vacuolar protein sorting 35 variant

349 (Fragment) OS=Homo sapiens PE=2 SV=1 Vasodilator-stimulated phosphoprotein

isoform 1 OS=Homo sapiens $\mathrm{GN}=\mathrm{VASP}$

$350 \quad P E=2 S V=1$

Signal transducer and activator of

transcription (Fragment) OS=Homo

351 sapiens $P E=2 S V=1$

Septin 6, isoform CRA_b OS=Homo

352 sapiens $\mathrm{GN}=\mathrm{SEPT} 6 \mathrm{PE}=1 \mathrm{SV}=1$

Insulin-like growth factor-binding protein

complex acid labile subunit OS=Homo

353 sapiens $\mathrm{GN}=$ IGFALS $P E=1 \mathrm{SV}=1$

Sushi, von Willebrand factor type A, EGF

and pentraxin domain-containing protein 1

$\mathrm{OS}=$ Homo sapiens $\mathrm{GN}=\mathrm{SVEP} 1 \mathrm{PE}=1$

$354 \quad S V=1$

EMILIN-1 OS=Homo sapiens

355 GN=EMILIN1 PE=1 SV=1

Ras GTPase-activating protein 3

$\mathrm{OS}=$ Homo sapiens $\mathrm{GN}=\mathrm{RASA} 3 \mathrm{PE}=1$

$356 \quad$ SV $=3$

Glycyl-tRNA synthetase OS=Homo

357 sapiens $\mathrm{GN}=\mathrm{GARS} \mathrm{PE}=4 \mathrm{SV}=1$

cDNA FLJ53218, highly similar to Homo

sapiens SAC1 suppressor of actin

mutations 1-like (SACM1L), mRNA

358 OS=Homo sapiens $P E=2$ SV=1

Collagen alpha- $3(\mathrm{VI})$ chain $\mathrm{OS}=\mathrm{Homo}$

359 sapiens $\mathrm{GN}=\mathrm{COL} 6 \mathrm{~A} 3 \mathrm{PE}=1 \mathrm{SV}=5$

$=1$

A8K310 HUMAN $(+4)$

GSTP1_HUMAN (+1)

$83 \quad 0.026 \quad 0.000$

0.000

0.000

S6B2B6_HUMAN

B4DUV1_HUMAN

$\begin{array}{lll}23 & 0.046 \quad 0.000\end{array}$

0.000

$0.000 \quad 0.000$

$\begin{array}{lll}29 & 0.000 & 0.000\end{array}$

$\begin{array}{lll}0.000 & 0.000 & 0.046 \\ 0.000 & 0.000 & 0.000\end{array}$

$\begin{array}{lll}70 & 0.000 & 0.046\end{array}$

BOYJ73_HUMAN (+1)

$\begin{array}{lll}26 & 0.000 & 0.000\end{array}$

0.000

$0.000 \quad 0.000$

$\begin{array}{lll}127 & 0.017 & 0.000\end{array}$

0.000

$0.000 \quad 0.000$

PDLI1_HUMAN (+1)

$\begin{array}{lll}36 & 0.044 & 0.000\end{array}$

0.000

$0.000 \quad 0.000$

0.000

$92 \quad 0.012$

0.010

0.000

0.01

A0A024ROV4_HUMAN (+2)

$\begin{array}{lll}40 & 0.000 & 0.000\end{array}$

0.000

$0.000 \quad 0.000$

Q59H39 HUMAN (+1)

$\begin{array}{lll}90 & 0.018 & 0.000\end{array}$

0.000

$0.000 \quad 0.000$

B1AMS2_HUMAN (+9)

$49 \quad 0.043$

0.000

0.000

0.000

ALS_HUMAN (+2)

66

0.000

0.000

0.043

0.000

AOAOAOMSDO HUMAN (+1)

$\begin{array}{lll}390 & 0.038 & 0.000\end{array}$

0.002

0.000

0.002

A0A0C4DFX3_HUMAN (+1)

$\begin{array}{lll}107 & 0.010 & 0.000\end{array}$

0.000

$\begin{array}{lll}0.000 & 0.000 \quad 0.033\end{array}$

0.000

0.000

0.043

0.000

0.00

A0A090N8G0_HUMAN (+1)

$\begin{array}{lll}78 & 0.027 & 0.000\end{array}$

0.000

B4DVV3_HUMAN (+2)

56

0.000

0.000

0.000

0.000

0.000

0.042

0.007

CO6A3_HUMAN (+1)

344

0.00

0.004

0.000

0.00

0.00
0.026

$0.007 \quad 0.010$ 
$4 \quad$ Platelet endothelial cell adhesion molecule

$5 \quad$ OS=Homo sapiens GN=PECAM1 PE=1

8

Solute carrier family 2, facilitated glucose

transporter member $14 \mathrm{OS}=$ Homo sapiens

$\mathrm{GN}=\mathrm{SLC2A} 14 \mathrm{PE}=2 \mathrm{SV}=1$

cDNA, FLJ96812, highly similar to Homo

sapiens threonyl-tRNA synthetase (TARS)

mRNA OS=Homo sapiens $P E=1 \mathrm{SV}=1$

Serine/threonine-protein phosphatase $2 \mathrm{~A}$

$65 \mathrm{kDa}$ regulatory subunit $A$ alpha isoform

OS=Homo sapiens $\mathrm{GN}=\mathrm{PPP} 2 \mathrm{R} 1 \mathrm{~A} P \mathrm{PE}=1$

UDP-glucose:glycoprotein

glucosyltransferase $1 \mathrm{OS}=\mathrm{Homo}$ sapiens

1 antiproteinase, antitrypsin), member 4 ,

isoform CRA_a OS=Homo sapiens

365 GN=SERPINA4 PE=3 SV=1

6-phosphogluconate dehydrogenase

decarboxylating OS=Homo sapiens

A0A075B738_HUMAN (+2)

GTR14_HUMAN (+1)

B2RDX5_HUMAN (+2)

2AAA_HUMAN $(+5)$

UGGG1_HUMAN

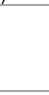



$A O A$

A0A024R6I9_HUMAN (+2)

$49 \quad 0.000$

0.019 0.000

$\mathrm{GN}=P G D \mathrm{PE}=1 \mathrm{SV}=3$

Dynamin-2 OS=Homo sapiens GN=DNM2

$367 \quad \mathrm{PE}=1 \mathrm{SV}=2$

Myosin light chain kinase, smooth muscle

368 OS=Homo sapiens $\mathrm{GN}=\mathrm{MYLK} P E=1 \mathrm{SV}=4$

Calnexin OS=Homo sapiens $\mathrm{GN}=\mathrm{CANX}$

$369 \quad \mathrm{PE}=1 \mathrm{SV}=2$

Lipopolysaccharide-responsive and beige-

like anchor protein OS=Homo sapiens

370 GN=LRBA PE=1 SV=1

Xaa-Pro aminopeptidase 1 OS=Homo

371 sapiens $\mathrm{GN}=\mathrm{XPNPEP} 1 \mathrm{PE}=1 \mathrm{SV}=3$

CD47 OS=Homo sapiens $G N=C D 47 P E=2$

$372 \quad S V=1$

cDNA FLJ59760, highly similar to 1,4

alpha-glucan branching enzyme (EC

373 2.4.1.18) OS=Homo sapiens PE=2 SV=1 Hepatocyte growth factor activator

OS=Homo sapiens $\mathrm{GN}=\mathrm{HGFAC} \mathrm{PE}=1$

374

$\mathrm{SV}=1$

cDNA, FLJ94267, highly similar to Homo

sapiens glutathione S-transferase omega 1

(GSTO1), mRNA OS=Homo sapiens PE=2

375 SV=1

Apolipoprotein A-V, isoform CRA_a

OS=Homo sapiens $\mathrm{GN}=\mathrm{APOA} 5 \mathrm{PE}=4$

$376 \quad$ SV $=1$

Platelet glycoprotein VI OS=Homo sapiens

377 GN=GP6 PE=1 SV=4

Aldehyde dehydrogenase family 16

member A1 OS=Homo sapiens

378 GN=ALDH16A1 PE=1 SV=2

$\mathrm{MHC}$ class I antigen (Fragment) OS=Hom

379 sapiens $\mathrm{GN}=\mathrm{HLA}-\mathrm{A} P \mathrm{PE}=3 \mathrm{SV}=1$

X-DING-CD4 (Fragment) OS=Homo

380 sapiens $P E=2$ SV=1

cDNA FLJ53963, highly similar to

Leukocyte elastase inhibitor OS=Homo

381 sapiens $P E=2$ SV=1

SLAM family member 5 OS=Homo sapiens

$382 \mathrm{GN}=\mathrm{CD} 84 \mathrm{PE}=1 \mathrm{SV}=1$

Tyrosine-protein kinase BTK OS=Homo

383 sapiens $\mathrm{GN}=\mathrm{BTK} \mathrm{PE}=1 \mathrm{SV}=3$

ATP synthase subunit alpha, mitochondrial

OS=Homo sapiens $G N=A T P 5 A 1 P E=1$

$384 \quad$ SV=1

Attractin OS=Homo sapiens $\mathrm{GN}=\mathrm{ATRN}$

385

$\mathrm{PE}=1 \mathrm{SV}=2$

Actin related protein $2 / 3$ complex, subunit

$1 \mathrm{~B}, 41 \mathrm{kDa}$ OS=Homo sapiens

386 GN=ARPC1B PE=2 SV=1

Coagulation factor VIII OS=Homo sapiens

$387 \quad \mathrm{GN}=\mathrm{F} 8 \mathrm{PE}=1 \mathrm{SV}=1$

ATP synthase subunit beta, mitochondrial

$\mathrm{OS}=$ Homo sapiens $\mathrm{GN}=\mathrm{ATP} 5 \mathrm{~B} \mathrm{PE}=1$

$388 \quad$ SV $=3$

6PGD_HUMAN (+1)

$53 \quad 0.020$

0.017

0.000

$0.000 \quad 0.000$

$\begin{array}{lll}98 & 0.011 & 0.000\end{array}$

0.000

$0.000 \quad 0.000$

MYLK HUMAN

$\begin{array}{lll}211 & 0.010 & 0.011\end{array}$

0.000

$0.000 \quad 0.000$

$\begin{array}{lll}68 & 0.000 & 0.000\end{array}$

0.000

0.000

CALX_HUMAN

$\begin{array}{lll}287 & 0.011 & 0.000\end{array}$

0.000

$0.000 \quad 0.000$

$\begin{array}{lll}70 & 0.000 & 0.000\end{array}$

0.000

XPP1_HUMAN

AOA0A1TSG4_HUMAN (+1)

$\begin{array}{lll}32 & 0.033 & 0.000\end{array}$

0.000

$0.000 \quad 0.000$

B4DUF1_HUMAN (+3)

$\begin{array}{ll}76 & 0.021\end{array}$

0.012

0.000

$\begin{array}{lll}0.000 & 0.000 & 0.000\end{array}$

$0.000 \quad 0.000$

0.000

$71 \quad 0.000$

0.019

0.013

0.000

0.000

0.000

$\begin{array}{lll}28 & 0.000 & 0.033\end{array}$

0.000

0.000

0.000

0.000

0.000

0.000

$0.032 \quad 0.000$

$\begin{array}{llll}37 & 0.000 & 0.000 & 0.000\end{array}$

0.000

GPVI_HUMAN

$85 \quad 0.000 \quad 0.011$

0.000

$0.000 \quad 0.000$

$\begin{array}{lll}38 & 0.000 & 0.000\end{array}$

0.000

$0.000 \quad 0.000$

$\begin{array}{lll}30 & 0.000 & 0.030\end{array}$

0.000

0.000

0.000

$\begin{array}{lll}39 & 0.000 & 0.000\end{array}$

$\begin{array}{lll}0.000 & 0.000 & 0.000\end{array}$

0.030

$\begin{array}{lll}39 & 0.000 & 0.000\end{array}$

0.000

SLAF5_HUMAN

BTK_HUMAN

$\begin{array}{lll}76 & 0.014 & 0.000\end{array}$

0.000

$0.000 \quad 0.000$

0.000

0.030

ATPA HUMAN (+1)

$\begin{array}{lll}60 & 0.000 \quad 0.000\end{array}$

0.000

$0.000 \quad 0.000$

0.029

ATRN_HUMAN (+1)

$159 \quad 0.000$

0.017

0.006

0.00

A4D275_HUMAN (+1)

$\begin{array}{lll}41 & 0.000 & 0.000\end{array}$

0.000

$0.000 \quad 0.000$

$\begin{array}{lll}267 & 0.010 & 0.000\end{array}$

0.007

0.00

ATPB_HUMAN (+3)
57
0.028
0.000

0.000

0.000

0.000 
\begin{tabular}{l|l}
\hline 4 & cDNA FLJ45348 fis, clone BRHIP3011269,
\end{tabular}

5 weakly similar to Prenylcysteine oxidase (EC 1.8.3.5) OS=Homo sapiens $\mathrm{PE}=2$

\begin{tabular}{ll|l}
6 & 389 & SV=1 \\
\hline 7 & & HMHA1 protein (Fragment) OS=Homo
\end{tabular}

8390 sapiens $\mathrm{GN}=\mathrm{HMHA1} \quad \mathrm{PE}=2 \mathrm{SV}=1$

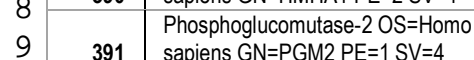

Protein 4.1 OS=Homo sapiens $G N=E P B 41$

$392 \quad \mathrm{PE}=1 \mathrm{SV}=4$

cDNA FLJ56358, highly similar to Cytosolic

nonspecific dipeptidase OS=Homo sapiens

$393 \mathrm{PE}=2 \mathrm{SV}=1$

Fatty acid synthase $\mathrm{OS}=$ Homo sapiens

Bromodomain adjacent to zinc finger

domain protein $2 \mathrm{~B} O \mathrm{OS}=$ Homo sapiens

$395 \mathrm{GN}=\mathrm{BAZ2B} \quad \mathrm{PE}=1 \mathrm{SV}=3$

Acyl-coenzyme A oxidase OS=Homo

ARP3 actin-related protein 3 homolog

(Yeast), isoform CRA_a OS=Homo

397 sapiens $G N=A C T R 3 \quad P E=3$ SV $=1$

Hypoxia up-regulated protein $1 \mathrm{OS}=\mathrm{Homo}$

398 sapiens $\mathrm{GN}=\mathrm{HYOU} 1 \mathrm{PE}=1 \mathrm{SV}=1$

Coatomer subunit beta' $\mathrm{OS}=$ Homo sapiens

$399 \mathrm{GN}=\mathrm{COPB} 2 \mathrm{PE}=1 \mathrm{SV}=2$

Nck-associated protein 1 OS=Homo

400 sapiens $G N=N C K A P 1 P E=1 \quad S V=1$

Carboxypeptidase B2 OS=Homo sapiens

$401 \quad \mathrm{GN}=\mathrm{CPB} 2 \mathrm{PE}=1 \mathrm{SV}=1$

ADAM metallopeptidase domain 10 ,

isoform CRA_b OS=Homo sapiens

$402 \mathrm{GN}=A D A M 10 \mathrm{PE}=4 \mathrm{SV}=1$

Complement factor properdin isoform 1

(Fragment) OS=Homo sapiens GN=CFP

$403 \quad \mathrm{PE}=2 \mathrm{SV}=1$

Neurobeachin-like protein $2 \mathrm{OS}=$ Homo

404 sapiens $\mathrm{GN}=\mathrm{NBEAL2} \mathrm{PE}=1 \mathrm{SV}=2$

Collagen alpha-2(VI) chain OS=Homo

405 sapiens $\mathrm{GN}=\mathrm{COL6A2} P E=1 \mathrm{SV}=4$

cDNA FLJ61564, highly similar to Plexin

domain-containing protein $2 \mathrm{OS}=\mathrm{Homo}$

406 sapiens $P E=2 \mathrm{SV}=1$

Kinesin-like protein KIF2A OS=Homo

407 sapiens GN=KIF2A PE=1 SV=3

Protein kinase C substrate $80 \mathrm{~K}-\mathrm{H}$, isoform

CRA_a OS=Homo sapiens $G N=P R K C S H$

$408 \quad \mathrm{PE}=4 \mathrm{SV}=1$

Coronin OS=Homo sapiens $\mathrm{GN}=\mathrm{CORO1B}$

$409 \quad P E=3 S V=1$

T-complex protein 1 subunit beta

410 OS=Homo sapiens $G N=C C T 2 P E=1 \quad S V=2$

cDNA FLJ76863, highly similar to Homo

sapiens stress-induced-phosphoprotein 1

(Hsp70/Hsp90-organizing protein) (STIP1),

411 mRNA OS=Homo sapiens $P E=2 S V=1$

Laminin, gamma 1 (Formerly LAMB2),

isoform CRA_a OS=Homo sapiens

$412 \mathrm{GN}=\mathrm{LAMC} 1 \mathrm{PE}=4 \mathrm{SV}=1$

B3KXF9_HUMAN (+2)

$\begin{array}{lll}42 & 0.000 \quad 0.000\end{array}$

0.000

$0.000 \quad 0.000$

0.028

$0.005 \quad 0.011$

Q8IYN3_HUMAN

$\begin{array}{lll}124 & 0.009 & 0.000\end{array}$

$\begin{array}{lll}0.000 & 0.000 \quad 0.000\end{array}$

0.019

PGM2_HUMAN

$\begin{array}{lll}68 & 0.000 & 0.000\end{array}$

0.000

0.000

0.000

0.026

0.00

41_HUMAN (+2)

$\begin{array}{lll}97 & 0.011 & 0.000\end{array}$

0.015

$\begin{array}{ll}0.000 & 0.000\end{array}$

B4DPF1_HUMAN (+2)

$42 \quad 0.025$

0.000

0.000

$0.000 \quad 0.000$

0.000

$0.004 \quad 0.010$

$\begin{array}{llllllllll}\text { AOAOU1RQFO_HUMAN (+1) } & 273 & 0.012 & 0.000 & 0.000 & 0.000 & 0.000 & 0.013 & 0.004 & 0.006\end{array}$

BAZ2B_HUMAN

$\begin{array}{lll}240 & 0.009 & 0.000\end{array}$

0.000

0.00

A0A024R8L7_HUMAN (+2)

$\begin{array}{lll}75 & 0.000 & 0.000\end{array}$

0.000

0.000

A0A024RAI1_HUMAN (+3)

$47 \quad 0.023$

0.000

0.000

$0.000 \quad 0.000$

0.000

$0.004 \quad 0.009$

A0A087X054_HUMAN (+2)

$105 \quad 0.000$

COPB2_HUMAN

$102 \quad 0.010-0.000$

0.000

0.00

NCKP1_HUMAN

$\begin{array}{lll}129 & 0.008 & 0.000\end{array}$

0.000

$0.000 \quad 0.000$

A0A087WSY5_HUMAN (+1)

$\begin{array}{lll}44 & 0.000 & 0.000\end{array}$

0.022

0.000

$0.000 \quad 0.000$

0.012

$0.004 \quad 0.009$

A0A024R5U5_HUMAN (+1)

$\begin{array}{lll}84 & 0.000 & 0.000\end{array}$

0.000

$0.000 \quad 0.000$

0.000

0.014

$0.004 \quad 0.006$

AOAOS2Z415_HUMAN (+1)

$\begin{array}{lll}51 & 0.021 & 0.000\end{array}$

0.000

0.000

$0.000 \quad 0.000$

0.000

0.021

$0.004 \quad 0.009$

NBEL2 HUMAN

$\begin{array}{lll}303 & 0.005 & 0.000\end{array}$

0.000

$0.000 \quad 0.000$

0.000

CO6A2_HUMAN

$\begin{array}{lll}109 & 0.010 & 0.000\end{array}$

0.000

$0.000 \quad 0.000$

0.000

0.016

0.011

(1)

B4E367_HUMAN (+1)

$\begin{array}{lll}58 & 0.000 & 0.000\end{array}$

0.000

$0.000 \quad 0.000$

0.020

$\begin{array}{ll}0.003 & 0.008\end{array}$

KIF2A_HUMAN

$\begin{array}{lll}80 & 0.020 & 0.000\end{array}$

0.000

0.000

0.000

A0A024R7F1 HUMAN $(+5)$

$\begin{array}{lll}59 & 0.000 & 0.000\end{array}$

0.000

$0.000 \quad 0.000$

0.020

$0.003 \quad 0.008$

A0A024R5K1_HUMAN (+5)

$\begin{array}{lll}54 & 0.020 & 0.000\end{array}$

0.000

$0.000 \quad 0.000$

0.000

0.003

$\begin{array}{lll}57 & 0.019 & 0.000\end{array}$

0.000

$0.000 \quad 0.000$

0.000

$0.003 \quad 0.008$

Coagulation factor IX OS=Homo sapiens

$413 \mathrm{GN}=F 9$ p22 $\mathrm{PE}=2 \mathrm{SV}=1$

A8K690_HUMAN (+2)

$\begin{array}{lll}63 & 0.000 \quad 0.000\end{array}$

0.000

0.000

0.000

0.019

$0.003 \quad 0.008$

A0A024R972_HUMAN (+1)

$\begin{array}{lll}174 & 0.006 & 0.000\end{array}$

0.000

$0.000 \quad 0.005$

0.007

$0.003 \quad 0.003$

F2RM37_HUMAN (+1)

$52 \quad 0.000$

0.018

0.000

0.00

Heparanase, isoform CRA_a OS=Homo

414 sapiens $\mathrm{GN}=\mathrm{HPSE} P E=4 \mathrm{SV}=1$

cDNA, FLJ95309, highly similar to Homo

sapiens adenosine monophosphate

deaminase 2 (isoform L)(AMPD2), mRNA

415 OS=Homo sapiens $P E=2$ SV=1

cDNA FLJ50778, highly similar to Protein

flightless- 1 homolog OS=Homo sapiens

416

$P E=2 S V=1$

A0A024RDB8_HUMAN (+2)

$61 \quad 0.017$

0.000

0.000

0.000

0.000

0.000

0.003

B2RB47_HUMAN

101

0.000

0.000

0.017

$\begin{array}{ll}0.003 & 0.007\end{array}$

B4DIXO_HUMAN (+1)

141

0.000

0.000

0.000

0.000

0.000

0.017

$0.003 \quad 0.007$

shedding aminopeptidase regulator,

isoform CRA_a OS=Homo sapiens

$417 \mathrm{GN}=\mathrm{ARTS}-1 \mathrm{PE}=4 \mathrm{SV}=1$

Phospholipid transfer protein, isoform

CRA_c OS=Homo sapiens GN=PLTP

$418 \quad P E=2$ SV=1

A0A024RAR8_HUMAN (+13)

107

0.000

0.000

0.000

0.000

0.000

0.016

$0.003 \quad 0.007$

B3KUE5_HUMAN (+2)

$\begin{array}{lll}57 & 0.000 \quad 0.016\end{array}$

0.000

0.00

0.000 
\begin{tabular}{l|l}
\hline & HLA-B associated transcript 5, isoform
\end{tabular}

5 CRA_b OS=Homo sapiens GN=ABHD16A

\section{6}

\section{7}

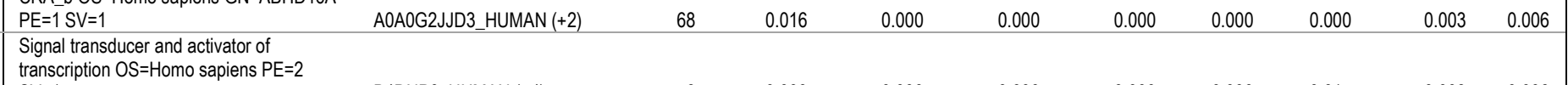

420 transcription OS=Homo sapiens $\mathrm{PE}=2$

ATPase $\mathrm{Ca}++$ transporting cardiac muscle

slow twitch 2 isoform 1 (Fragment)

OS=Homo sapiens $\mathrm{GN}=\mathrm{ATP} 2 \mathrm{~A} 2 \mathrm{PE}=2$

$421 \quad S V=1$

Glycerol-3-phosphate dehydrogenase

$\begin{array}{llllllllll}\text { B4DNPO_HUMAN (+4) } & 76 & 0.000 & 0.000 & 0.000 & 0.000 & 0.000 & 0.015 & 0.003 & 0.006\end{array}$

(Fragment) OS=Homo sapiens GN=GPD2

$422 \quad \mathrm{PE}=3 \mathrm{SV}=1$

$\mathrm{N}$-acylaminoacyl-peptide hydrolase

isoform CRA_b OS=Homo sapiens

423 GN=APEH PE=4 SV=1

Malic enzyme OS=Homo sapiens $\mathrm{PE}=2$

$424 \quad S V=1$

Integrin alpha-2 OS=Homo sapiens

425 GN=ITGA2 PE=1 SV=1

cDNA FLJ78440, highly similar to Human

426 lactoferrin $\mathrm{OS}=\mathrm{H}$ omo sapiens $\mathrm{PE}=2 \mathrm{SV}=1$

cDNA FLJ55918, highly similar to

Echinoderm microtubule-associated

protein-like $2 \mathrm{OS}=$ Homo sapiens $\mathrm{PE}=2$

$427 \quad \mathrm{SV}=1$

$\mathrm{NHL}$ repeat-containing protein $2 \mathrm{OS}=\mathrm{Homo}$

428 sapiens $\mathrm{GN}=\mathrm{NHLRC2} \mathrm{PE}=1 \mathrm{SV}=1$

Coagulation factor XII OS=Homo sapiens

$429 \mathrm{GN}=\mathrm{F} 12 \mathrm{PE}=4 \mathrm{SV}=1$

Aconitate hydratase, mitochondrial

430 OS=Homo sapiens $\mathrm{GN}=\mathrm{ACO} 2 \mathrm{PE}=1 \mathrm{SV}=1$

Carnitine palmitoyltransferase $1 \mathrm{~A}$ (Liver),

isoform CRA_a OS=Homo sapiens

431 GN=CPT1A PE=3 SV=1

cDNA FLJ50510, highly similar to Heat

shock $70 \mathrm{kDa}$ protein 4 OS=Homo sapiens

$432 \quad P E=2 S V=1$

Prolow-density lipoprotein receptor-related

protein $1 \mathrm{OS}=$ Homo sapiens $\mathrm{GN}=\mathrm{LRP} 1$

$433 \quad \mathrm{PE}=1 \mathrm{SV}=2$

Cytoplasmic aconitate hydratase

434 OS=Homo sapiens GN=AC01 PE=1 SV=3

cDNA FLJ77762, highly similar to Homo

sapiens cullin-associated and neddylation-

dissociated 1 (CAND1), mRNA OS=Homo

435 sapiens $P E=2 S V=1$

Phosphatidylinositol-glycan-specific

phospholipase D OS=Homo sapiens

$436 \mathrm{GN}=\mathrm{GPLD} 1 \mathrm{PE}=1 \mathrm{SV}=3$

cDNA FLJ51067, highly similar to DNA

damage-binding protein $1 \mathrm{OS}=\mathrm{Homo}$

437 sapiens $\mathrm{PE}=2 \mathrm{SV}=1$

Spectrin beta chain, non-erythrocytic 2

OS=Homo sapiens $\mathrm{GN}=\mathrm{SPTBN} 2 \mathrm{PE}=1$

$438 \quad$ SV=3

Epididymis luminal protein 102 OS=Homo

439 sapiens $\mathrm{GN}=\mathrm{SPTBN} 1 \mathrm{PE}=2 \mathrm{SV}=1$

Dynactin subunit $1 \mathrm{OS}=\mathrm{Homo}$ sapiens

$4 \quad \mathrm{GN}=\mathrm{DCTN} 1 \mathrm{PE}=1 \mathrm{SV}=3$

Laminin subunit beta-1 OS=Homo sapiens

$441 \mathrm{GN=LAMB1} P E=1 \mathrm{SV}=1$

Cytoplasmic dynein 1 heavy chain 1

OS=Homo sapiens GN=DYNC1H1 PE=1

$442 \quad S V=5$

Tenascin- $X$ OS=Homo sapiens GN=TNXB

$443 \quad P E=1 S V=1$

Hornerin OS=Homo sapiens GN=HRNR

$444 \quad \mathrm{PE}=1 \mathrm{SV}=2$

Hemicentin-1 OS=Homo sapiens

445 GN=HMCN1 PE=1 SV=2

AOA0S2Z3L2_HUMAN (+2)

$\begin{array}{lll}115 & 0.000 & 0.000\end{array}$

0.000

$0.000 \quad 0.000$

0.015

$0.003 \quad 0.006$

Q53T76_HUMAN

$\begin{array}{lll}77 & 0.000 & 0.000\end{array}$

0.000

$0.000 \quad 0.000$

0.015

$0.003 \quad 0.006$

A0A024R2U9_HUMAN (+2)

$\begin{array}{lll}81 & 0.000 & 0.000\end{array}$

A8K168_HUMAN (+1)

$\begin{array}{lll}64 & 0.000 & 0.014\end{array}$

0.000 $0.000 \quad 0.000$

0.015

$0.002 \quad 0.006$

ITA2_HUMAN

$\begin{array}{lll}129 & 0.000 & 0.000\end{array}$

0.000

$0.000 \quad 0.000$

A8K494_HUMAN (+8)

$\begin{array}{lll}78 & 0.014 & 0.000\end{array}$

0.000

0.000

0.000

0.000

$0.002 \quad 0.006$

B7Z2F5 HUMAN (+2)

NHLC2_HUMAN

A0A0R7FJH5_HUMAN (+2)

$\begin{array}{lll}87 & 0.000 & 0.000\end{array}$

0.000

$0.000 \quad 0.000$

0.014

$0.002 \quad 0.006$

A2A274_HUMAN (+7)

$\begin{array}{lll}79 & 0.013 & 0.000\end{array}$

$\begin{array}{lll}0.000 & 0.000 \quad 0.000\end{array}$

$\begin{array}{lll}0.000 & 0.002 & 0.006\end{array}$

A0A024R5F4_HUMAN (+2)

$\begin{array}{lll}68 & 0.000 & 0.013\end{array}$

0.000

$0.000 \quad 0.000$

0.000

$0.002 \quad 0.005$

B4DH02_HUMAN (+3)

$\begin{array}{lll}88 & 0.000 & 0.000\end{array}$

0.000

$0.000 \quad 0.000$

0.013

$0.002 \quad 0.005$

LRP1_HUMAN

$\begin{array}{lll}94 & 0.000 & 0.000\end{array}$

0.000

$0.000 \quad 0.000$

0.013

$0.002 \quad 0.005$

ACOC HUMAN (+1)

$505 \quad 0.004 \quad 0.002$

$0.000 \quad 0.000 \quad 0.003$

0.003

$0.002 \quad 0.002$

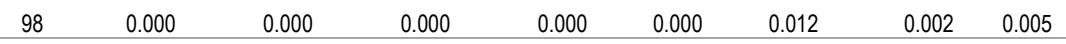

A8K8U1_HUMAN (+1)

$\begin{array}{lll}136 & 0.012 & 0.000\end{array}$

0.000

PHLD_HUMAN

$\begin{array}{lll}92 & 0.000 & 0.000\end{array}$

0.010

B4DSA8 HUMAN (+3)

$\begin{array}{lll}112 & 0.010 \quad 0.000\end{array}$

0.000

0.000

0.000

0.000

$0.002 \quad 0.004$

SPTN2_HUMAN

$271 \quad 0.000$

0.000

0.009

$0.000 \quad 0.000$

B2ZZ89_HUMAN (+2)

$\begin{array}{lll}275 & 0.000 & 0.000\end{array}$

0.000

$0.000 \quad 0.000$

0.000

0.009

DCTN1_HUMAN (+2)

G3XAI2_HUMAN (+1)

$\begin{array}{lll}142 & 0.000 & 0.000\end{array}$

0.000

$0.000 \quad 0.000$

0.000

0.008

0.00

$0.001 \quad 0.003$

DYHC1 HUMAN

200

\begin{tabular}{lccccccrrrr} 
DYHC1_HUMAN & 532 & 0.006 & 0.000 & 0.000 & 0.000 & 0.000 & 0.000 & 0.001 & 0.002 \\
A0A087X0I0_HUMAN (+2) & 213 & 0.000 & 0.000 & 0.000 & 0.000 & 0.000 & 0.006 & 0.001 & 0.002 \\
HORN_HUMAN & 282 & 0.000 & 0.000 & 0.000 & 0.003 & 0.000 & 0.000 & 0.001 & 0.001 \\
\hline HMCN1_HUMAN & 613 & 0.002 & 0.000 & 0.000 & 0.000 & 0.000 & 0.000 & 0.000 & 0.001
\end{tabular}


Table S4: Blood-circulation proteome analysis. Full list of proteins identified in plasma samples recovered from 6 ovarian carcinoma patients (across all samples).

\begin{tabular}{|c|c|c|c|c|c|c|c|c|c|c|c|}
\hline \# & Identified Proteins & Accession Number & MW & $\begin{array}{l}\text { RPA\% } \\
\text { (P1) }\end{array}$ & $\begin{array}{l}\mathrm{RPA} \% \\
(\mathrm{P} 2)\end{array}$ & $\begin{array}{l}\mathrm{RPA} \% \\
(\mathrm{P} 3)\end{array}$ & $\begin{array}{l}\text { RPA\% } \\
\text { (P4) }\end{array}$ & $\begin{array}{l}\text { RPA } \% \\
\text { (P5) }\end{array}$ & $\begin{array}{l}\text { RPA\% } \\
(\mathrm{P} 6)\end{array}$ & $\begin{array}{l}\text { Average } \\
\text { RPA\% }\end{array}$ & $\begin{array}{l}\text { Standard } \\
\text { Deviation }\end{array}$ \\
\hline 1 & $\begin{array}{l}\text { Serum albumin OS=Homo sapiens } G N=A L B \\
P E=1 S V=2\end{array}$ & ALBU_HUMAN & 69 & 5.712 & 8.072 & 7.063 & 9.004 & 7.367 & 14.689 & 8.651 & 3.154 \\
\hline 2 & $\begin{array}{l}\text { Putative uncharacterized protein } \\
\text { DKFZp686C11235 OS=Homo sapiens } \\
\text { GN=DKFZp686C11235 PE=1 SV=1 }\end{array}$ & Q6MZV7_HUMAN & 52 & 2.575 & 3.134 & 2.616 & 3.189 & 2.506 & 2.863 & 2.814 & 0.295 \\
\hline 3 & $\begin{array}{l}\text { IGH@ protein OS=Homo sapiens GN=IGH@ } \\
\text { PE=1 SV=1 }\end{array}$ & Q6GMX6_HUMAN & 51 & 2.195 & 2.551 & 2.253 & 2.503 & 2.837 & 2.488 & 2.471 & 0.230 \\
\hline 4 & $\begin{array}{l}\text { Putative uncharacterized protein } \\
\text { DKFZp686I04196 (Fragment) OS=Homo } \\
\text { sapiens GN=DKFZp686I04196 PE=1 SV=1 }\end{array}$ & Q6N093_HUMAN & 46 & 2.030 & 3.134 & 2.077 & 2.659 & 2.218 & 2.532 & 2.442 & 0.421 \\
\hline 5 & $\begin{array}{l}\text { Ig gamma-1 chain } \mathrm{C} \text { region OS=Homo sapiens } \\
\mathrm{GN}=I \mathrm{GHG} 1 \mathrm{PE}=1 \mathrm{SV}=1\end{array}$ & A0A087WV47_HUMAN (+1) & 51 & 2.220 & 2.459 & 2.184 & 2.455 & 2.746 & 2.424 & 2.415 & 0.202 \\
\hline 6 & cDNA FLJ78387 OS=Homo sapiens PE=1 SV=1 & A8K008_HUMAN & 52 & 2.283 & 2.420 & 2.142 & 2.473 & 2.702 & 2.451 & 2.412 & 0.189 \\
\hline 7 & $\begin{array}{l}\text { Transferrin variant (Fragment) OS=Homo } \\
\text { sapiens } P E=2 S V=1\end{array}$ & Q53H26_HUMAN & 77 & 1.810 & 2.250 & 1.807 & 2.637 & 2.602 & 2.911 & 2.336 & 0.459 \\
\hline 8 & $\begin{array}{l}\text { Putative uncharacterized protein } \\
\text { DKFZp686C15213 OS=Homo sapiens } \\
\text { GN=DKFZp686C15213 PE=1 SV=1 }\end{array}$ & Q6MZU6_HUMAN & 51 & 1.855 & 2.855 & 1.890 & 2.408 & 1.928 & 2.187 & 2.187 & 0.390 \\
\hline 9 & $\begin{array}{l}\text { Haptoglobin OS=Homo sapiens GN=HP PE=1 } \\
\text { SV=4 }\end{array}$ & HOY300_HUMAN & 49 & 2.319 & 1.639 & 2.471 & 1.875 & 2.006 & 2.433 & 2.124 & 0.336 \\
\hline 10 & $\begin{array}{l}\text { Putative uncharacterized protein } \\
\text { DKFZp686G11190 OS=Homo sapiens } \\
\text { GN=DKFZp686G11190 PE=1 SV=1 }\end{array}$ & Q6MZQ6_HUMAN & 52 & 1.860 & 2.023 & 1.820 & 2.110 & 2.399 & 1.976 & 2.031 & 0.209 \\
\hline 11 & $\begin{array}{l}\text { Ig gamma-3 chain } \mathrm{C} \text { region } \mathrm{OS}=\text { Homo sapiens } \\
\mathrm{GN}=1 \mathrm{GHG} 3 \mathrm{PE}=4 \mathrm{SV}=1\end{array}$ & A0A087WXL8_HUMAN & 57 & 1.808 & 2.290 & 1.776 & 2.273 & 1.676 & 2.274 & 2.016 & 0.291 \\
\hline 12 & $\begin{array}{l}\text { Haptoglobin (Fragment) OS=Homo sapiens } \\
\text { GN=HP PE=1 SV=1 }\end{array}$ & H3BS21_HUMAN & 25 & 2.332 & 1.540 & 2.289 & 1.508 & 1.744 & 2.461 & 1.979 & 0.429 \\
\hline 13 & $\begin{array}{l}\text { Fibrinogen alpha chain OS=Homo sapiens } \\
\mathrm{GN}=\mathrm{FGA} P E=1 \mathrm{SV}=2\end{array}$ & FIBA_HUMAN & 95 & 1.819 & 1.720 & 2.368 & 2.092 & 1.694 & 2.035 & 1.955 & 0.260 \\
\hline 14 & $\begin{array}{l}\text { Ig gamma-1 chain } C \text { region OS=Homo sapiens } \\
G N=I G H G 1 P E=1 S V=1\end{array}$ & A0A087WYC5_HUMAN & 52 & 2.120 & 2.330 & 2.083 & 2.362 & 2.613 & 0.000 & 1.918 & 0.959 \\
\hline 15 & $\begin{array}{l}\text { Complement } \mathrm{C} 3 \mathrm{OS}=\mathrm{Homo} \text { sapiens } \mathrm{GN}=\mathrm{C} 3 \\
\mathrm{PE}=1 \mathrm{SV}=2\end{array}$ & CO3_HUMAN (+1) & 187 & 1.927 & 2.207 & 1.820 & 1.536 & 1.835 & 1.889 & 1.869 & 0.216 \\
\hline 16 & $\begin{array}{l}\text { Alpha-2-macroglobulin OS=Homo sapiens } \\
\text { GN=A2M PE=1 SV=3 }\end{array}$ & A2MG_HUMAN & 163 & 1.514 & 1.740 & 1.007 & 1.667 & 2.265 & 1.715 & 1.651 & 0.406 \\
\hline 17 & $\begin{array}{l}\text { Fibrinogen beta chain OS=Homo sapiens } \\
\mathrm{GN}=\mathrm{FGB} P E=1 \mathrm{SV}=2\end{array}$ & FIBB_HUMAN (+1) & 56 & 1.441 & 1.291 & 1.667 & 1.537 & 1.259 & 1.707 & 1.484 & 0.187 \\
\hline 18 & $\begin{array}{l}\text { Lambda-chain (AA }-20 \text { to } 215) \text { OS=Homo } \\
\text { sapiens } P E=1 S V=1\end{array}$ & A2NUT2_HUMAN & 25 & 1.284 & 1.446 & 1.479 & 1.489 & 1.169 & 1.362 & 1.372 & 0.126 \\
\hline 19 & $\begin{array}{l}\text { IGL@ protein OS=Homo sapiens GN=IGL@ } \\
\text { PE=2 SV=1 }\end{array}$ & Q6PIQ7_HUMAN & 25 & 1.234 & 1.465 & 1.550 & 1.392 & 1.113 & 1.340 & 1.349 & 0.158 \\
\hline 20 & $\begin{array}{l}\text { IGL@ protein OS=Homo sapiens GN=IGL@ } \\
\text { PE=1 SV=1 }\end{array}$ & Q8N355_HUMAN & 25 & 1.267 & 1.446 & 1.444 & 1.431 & 1.132 & 1.340 & 1.343 & 0.126 \\
\hline 21 & $\begin{array}{l}\text { Protein IGHV3-72 OS=Homo sapiens } \\
\mathrm{GN}=I \mathrm{GHV} 3-72 \mathrm{PE}=4 \mathrm{SV}=1\end{array}$ & A0A087WW89_HUMAN & 11 & 1.421 & 1.195 & 1.161 & 1.538 & 1.265 & 1.199 & 1.296 & 0.150 \\
\hline 22 & $\begin{array}{l}\text { cDNA FLJ14473 fis, clone MAMMA1001080, } \\
\text { highly similar to Homo sapiens SNC73 protein } \\
\text { (SNC73) mRNA OS=Homo sapiens PE=2 SV=1 }\end{array}$ & Q96K68_HUMAN & 53 & 1.172 & 1.630 & 1.096 & 1.286 & 0.980 & 1.337 & 1.250 & 0.226 \\
\hline 23 & $\begin{array}{l}\text { Alpha-1-antitrypsin OS=Homo sapiens } \\
\text { GN=SERPINA1 PE=1 SV=3 }\end{array}$ & A1AT_HUMAN (+1) & 47 & 1.402 & 1.339 & 0.965 & 1.173 & 1.283 & 1.169 & 1.222 & 0.156 \\
\hline 24 & $\begin{array}{l}\text { Putative uncharacterized protein } \\
\text { DKFZp686P15220 OS=Homo sapiens } \\
\text { GN=DKFZp686P15220 PE=1 SV=1 }\end{array}$ & Q6N089_HUMAN & 52 & 2.120 & 2.348 & 0.000 & 0.000 & 2.622 & 0.000 & 1.182 & 1.304 \\
\hline 25 & $\begin{array}{l}\text { Ig gamma- } 4 \text { chain } C \text { region OS=Homo sapiens } \\
\mathrm{GN}=I \mathrm{GHG} 4 \mathrm{PE}=1 \mathrm{SV}=1\end{array}$ & IGHG4_HUMAN (+1) & 36 & 1.127 & 1.370 & 0.905 & 1.061 & 1.494 & 1.099 & 1.176 & 0.216 \\
\hline 26 & $\begin{array}{l}\text { Haptoglobin-related protein OS=Homo sapiens } \\
\text { GN=HPR PE=1 SV=2 }\end{array}$ & HPTR_HUMAN & 39 & 1.376 & 1.048 & 1.287 & 0.992 & 1.035 & 1.183 & 1.153 & 0.155 \\
\hline 27 & IgG H chain OS=Homo sapiens $P E=1 S V=1$ & S6B291_HUMAN & 51 & 2.137 & 0.000 & 2.115 & 0.000 & 2.664 & 0.000 & 1.153 & 1.278 \\
\hline 28 & $\begin{array}{l}\text { Fibrinogen gamma chain OS=Homo sapiens } \\
G N=F G G P E=1 S V=3\end{array}$ & FIBG_HUMAN & 52 & 1.056 & 0.984 & 1.287 & 1.162 & 0.972 & 1.109 & 1.095 & 0.119 \\
\hline 29 & IgG $L$ chain $O S=$ Homo sapiens $P E=1 \mathrm{SV}=1$ & S6BGD6_HUMAN & 25 & 1.048 & 1.165 & 1.215 & 1.180 & 0.890 & 1.033 & 1.088 & 0.122 \\
\hline 30 & $\begin{array}{l}\text { Ig mu chain } C \text { region } O S=\text { Homo sapiens } \\
G N=I G H M P E=1 S V=1\end{array}$ & A0A087X2C0_HUMAN & 64 & 0.937 & 0.903 & 1.107 & 1.065 & 1.536 & 0.764 & 1.052 & 0.267 \\
\hline
\end{tabular}


Immunoglobulin heavy chain variant (Fragment) $\mathrm{OS}=$ Homo sapiens $\mathrm{PE}=2 \mathrm{SV}=1$
Hemopexin OS=Homo sapiens GN=HPX PE=1 $32 \mathrm{SV}=2$

33 Ig $\mathrm{H}$ chain $\mathrm{OS}=$ Homo sapiens $\mathrm{PE}=2 \mathrm{SV}=1$ Apolipoprotein $B$ (Including $\mathrm{Ag}(\mathrm{X})$ antigen)

34 OS=Homo sapiens $\mathrm{GN}=\mathrm{APOB} P E=4 \mathrm{SV}=1$ Ig mu chain $\mathrm{C}$ region OS=Homo sapiens

$G N=I G H M P E=1 \quad S V=1$

Epididymis tissue protein Li 173 OS=Homo

sapiens $\mathrm{PE}=2 \mathrm{SV}=1$

IGL@ protein OS=Homo sapiens GN=IGL@

$P E=1 \quad S V=1$

Q9NPP6_HUMAN

$\begin{array}{llll}45 & 0.967 & 1.325 & 0.910\end{array}$

HEMO_HUMAN

\begin{tabular}{llll}
52 & 0.894 & 1.102 & 1.075 \\
\hline
\end{tabular}

0.999

$0.866 \quad 1.086$

1.026

0.165

S6BGEO_HUMAN

$\begin{array}{llll}32 & 1.162 & 1.247 & 1.059\end{array}$

0.948

$\begin{array}{ll}0.865 & 1.225 \\ 1.043 & 0.000\end{array}$

0.018
0.139

COJYY2_HUMAN

516

$0.736 \quad 1.033$

0.672

0.840

$1.211 \quad 0.942$

$\begin{array}{llllllllll}\text { A0A087WYJ9_HUMAN } & 66 & 0.877 & 0.904 & 1.101 & 1.048 & 1.497 & 0.000 & 0.904 & 0.496\end{array}$

$\begin{array}{llllllllll}\text { E9KL26_HUMAN (+1) } & 55 & 1.014 & 0.990 & 0.776 & 0.765 & 0.902 & 0.879 & 0.888 & 0.104\end{array}$

Q6PIK1_HUMAN

$25 \quad 0.828$

$0.990 \quad 0.776$

0.765

$\begin{array}{ll}0.902 & 0.879 \\ 0.668 & 0.879\end{array}$

0.881

0.130

Myosin-reactive immunoglobulin heavy chain

41 Serotransferrin OS=Homo sapiens $P E=2 S V=1$

Ig gamma- 1 chain $\mathrm{C}$ region $\mathrm{OS}=$ Homo sapiens

GN=IGHG1 PE=1 SV=1

Epididymis secretory protein Li 51 OS=Homo

sapiens $\mathrm{GN}=H E L-S-51 \mathrm{PE}=2 \mathrm{SV}=1$

Kininogen 1, isoform CRA a OS=Homo sapiens

$\mathrm{GN}=\mathrm{KNG} 1 \mathrm{PE}=4 \mathrm{SV}=1$

Plasminogen OS=Homo sapiens $G N=P L G$

$P E=1 S V=2$

Alpha-2 globin chain OS=Homo sapiens

$\mathrm{GN}=\mathrm{HBA} 2 \mathrm{PE}=3 \mathrm{SV}=1$

Vitronectin OS=Homo sapiens GN=VTN PE $=4$

SV=1

Inter-alpha (Globulin) inhibitor $\mathrm{H} 4$ (Plasma

Kallikrein-sensitive glycoprotein) OS=Homo

48 sapiens $\mathrm{GN}=I T \mid H 4 \mathrm{PE}=2 \mathrm{SV}=1$

Complement C4-B OS=Homo sapiens $\mathrm{GN}=\mathrm{C} 4 \mathrm{~B}$

$\mathrm{PE}=1 \mathrm{SV}=2$

Alpha-1-acid glycoprotein 1 OS=Homo sapiens

$G N=O R M 1 P E=1 S V=1$

Inter-alpha-trypsin inhibitor heavy chain $\mathrm{H} 2$

$51 \mathrm{OS}=$ Homo sapiens $\mathrm{GN}=\mathrm{IT} \mid \mathrm{H} 2 \mathrm{PE}=1 \mathrm{SV}=1$

Complement factor $\mathrm{HOS}=$ Homo sapiens

$52 \mathrm{GN}=\mathrm{CFH} P E=1 \mathrm{SV}=4$

Ig kappa chain V-III region WOL OS=Homo

sapiens $\mathrm{PE}=1 \mathrm{SV}=1$

Complement C4-A OS=Homo sapiens $\mathrm{GN}=\mathrm{C} 4 \mathrm{~A}$

$54 \quad \mathrm{PE}=1 \mathrm{SV}=2$

Inter-alpha-trypsin inhibitor heavy chain $\mathrm{H} 1$

$\mathrm{OS}=$ Homo sapiens $\mathrm{GN}=\mathrm{ITIH} 1 \mathrm{PE}=1 \mathrm{SV}=3$

$\mathrm{CP}$ protein $\mathrm{OS}=$ Homo sapiens $\mathrm{GN}=\mathrm{CP} \mathrm{PE}=2$

$56 \quad \mathrm{SV}=1$

$\mathrm{SV}=1$
cDNA FLJ35730 fis, clone TESTI2003131,

Q9UL88_HUMAN

\begin{tabular}{llll}
14 & 1.328 & 0.772 & 0.692 \\
\hline
\end{tabular}

1.036

0.596

0.824

0.875

0.267

Q6PIL8 HUMAN

$\begin{array}{llll}26 & 0.780 & 0.993 & 0.897\end{array}$

0.837

$\begin{array}{lll}0.910 & 0.676 & 0.849\end{array}$

0.111

A0A087X1C7_HUMAN

$\begin{array}{llll}50 & 0.000 & 0.000 & 2.148\end{array}$

$\begin{array}{lll}0.000 & 2.699 & 0.000\end{array}$

0.808

1.264

B4E1B2_HUMAN

$\begin{array}{llll}75 & 1.870 & 0.000 & 0.000\end{array}$

2.746

$\begin{array}{lll}0.000 & 0.000 & 0.769\end{array}$

1.224

A0A087X079 HUMAN

$\begin{array}{llll}52 & 2.161 & 0.000 & 0.000\end{array}$

$\begin{array}{llll}2.399 & 0.000 & 0.000 & 0.760\end{array}$

1.180

V9HWI6_HUMAN (+1)

$\begin{array}{llll}53 & 0.630 & 0.682 & 0.581\end{array}$

0.648

$0.543 \quad 0.974$

0.676

0.154

D3DNU8_HUMAN

$\begin{array}{llll}48 & 0.493 & 0.587 & 0.688\end{array}$

0.584

PLMN_HUMAN

$\begin{array}{llll}91 & 0.613 & 0.578 & 0.542\end{array}$

0.473

$\begin{array}{lll}0.580 & 0.858 & 0.632\end{array}$

0.127

D1MGQ2_HUMAN (+2)

$15 \quad 0.25$

$0.253 \quad 0.657$

$0.657 \quad 1.379$

0.548

$0.571 \quad 1.008$

0.631

0.191

D9ZGG2_HUMAN (+1)

$\begin{array}{llll}54 & 0.634 & 0.557 & 0.660\end{array}$

0.537

$\begin{array}{ll}0.495 & 0.330 \\ 0.558 & 0.692\end{array}$

0.610 0.404

B2RMS9_HUMAN (+1)

$\begin{array}{llll}103 & 0.578 & 0.675 & 0.603\end{array}$

0.530

0.558

$0.692 \quad 0.606$

0.064

CO4B_HUMAN

$\begin{array}{llll}193 & 0.560 & 0.749 & 0.586\end{array}$

0.441

$0.698 \quad 0.533$

0.603 0.071

A1AG1_HUMAN (+1)

$\begin{array}{llllllll}24 & 0.792 & 0.528 & 0.715 & 0.544 & 0.406 & 0.504 & 0.582\end{array}$

Q5T985_HUMAN

$\begin{array}{llll}105 & 0.539 & 0.599 & 0.495\end{array}$

0.529

$0.663 \quad 0.659$

0.581
0.144

CFAH_HUMAN

$\begin{array}{lllll}139 & 0.538 & 0.530 & 0.557 & 0.390\end{array}$

$0.574 \quad 0.830$

0.570

0.071

KV305_HUMAN

$\begin{array}{lllllllll}12 & 0.493 & 0.626 & 0.404 & 0.604 & 0.541 & 0.595 & 0.544\end{array}$
0.143

CO4A_HUMAN

$\begin{array}{llllllll}193 & 0.552 & 0.749 & 0.591 & 0.000 & 0.598 & 0.646 & 0.523\end{array}$

ITIH1_HUMAN

$\begin{array}{llllllll}101 & 0.489 & 0.590 & 0.466 & 0.469 & 0.652 & 0.451 & 0.520\end{array}$

0.520

A5PL27_HUMAN (+1)

$\begin{array}{llll}122 & 0.381 & 0.620 & 0.476\end{array}$

0.472

$\begin{array}{lll}0.566 & 0.572 & 0.514\end{array}$

highly similar to ALPHA-1.

ANTICHYMOTRYPSIN OS=Homo sapiens

$57 \quad \mathrm{PE}=2 \mathrm{SV}=1$

IBM-B2 heavy chain variable region (Fragment)

$O S=$ Homo sapiens $P E=2 \quad S V=1$

Prothrombin OS=Homo sapiens $\mathrm{GN}=\mathrm{F} 2 \mathrm{PE}=1$

$59 \quad \mathrm{SV}=2$

Myosin-reactive immunoglobulin heavy chain

variable region (Fragment) OS=Homo sapiens

$60 \quad \mathrm{PE}=2 \mathrm{SV}=1$

Putative uncharacterized protein

DKFZp686L19235 OS=Homo sapiens

$61 \mathrm{GN}=\mathrm{DKFZ}$ 686L19235 PE=2 SV=1

Alpha-1B-glycoprotein OS=Homo sapiens

$62 \mathrm{GN}=\mathrm{A} 1 \mathrm{BG} \quad \mathrm{PE}=1 \mathrm{SV}=4$

APOB protein OS=Homo sapiens $G N=A P O B$

$63 \quad \mathrm{PE}=2 \mathrm{SV}=1$

Heparin cofactor 2 OS=Homo sapiens

$64 \mathrm{GN}=\mathrm{SERPIND} 1 \mathrm{PE}=1 \mathrm{SV}=3$

cDNA, FLJ93914, highly similar to Homo

sapiens histidine-rich glycoprotein (HRG),

65 mRNA OS=Homo sapiens $P E=2 \quad S V=1$

$\begin{array}{lccccccccc}\text { B3KS79_HUMAN } & 51 & 0.646 & 0.562 & 0.518 & 0.379 & 0.546 & 0.399 & 0.508 & 0.102 \\ \text { A0A125QYY9_HUMAN } & 14 & 0.422 & 0.570 & 0.472 & 0.587 & 0.563 & 0.392 & 0.501 & 0.084 \\ \text { THRB_HUMAN } & 70 & 0.416 & 0.429 & 0.296 & 0.435 & 0.384 & 0.738 & 0.450 & 0.150 \\ \text { Q9UL90_HUMAN } & 12 & 0.669 & 0.509 & 0.367 & 0.483 & 0.309 & 0.275 & 0.435 & 0.147 \\ & & & & & & & & & \\ \text { Q6MZV6_HUMAN } & 52 & 0.886 & 0.000 & 0.838 & 0.000 & 0.803 & 0.000 & 0.421 & 0.462 \\ \text { A1BG_HUMAN (+1) } & 54 & 0.391 & 0.487 & 0.399 & 0.233 & 0.421 & 0.498 & 0.405 & 0.095 \\ \text { Q7Z7Q0_HUMAN } & 92 & 0.000 & 0.000 & 0.000 & 1.030 & 0.000 & 1.290 & 0.387 & 0.605 \\ \text { HEP2_HUMAN } & 57 & 0.378 & 0.379 & 0.332 & 0.441 & 0.415 & 0.357 & 0.384 & 0.039 \\ \text { B2R812_HUMAN } & & & & & & & & & \\ & 60 & 0.437 & 0.368 & 0.367 & 0.298 & 0.332 & 0.449 & 0.375 & 0.058\end{array}$


Apolipoprotein A-I, isoform CRA_a OS=Homo sapiens $\mathrm{GN}=\mathrm{APOA} 1 \mathrm{PE}=4 \mathrm{SV}=1$

A0A024R3E3 HUMAN (+1)

$\begin{array}{lll}0.313 & 0.364 & 0.284\end{array}$

0.421

0.509

0.319

0.368

0.084

Myosin-reactive immunoglobulin light chain

variable region (Fragment) OS=Homo sapiens

$67 \quad \mathrm{PE}=1 \mathrm{SV}=1$

Q9UL 83 HUMAN

$\begin{array}{lll}0.317 & 0.352 & 0.330\end{array}$

0.524

$0.271 \quad 0.366$

0.360

0.087

Hemoglobin, beta OS=Homo sapiens $\mathrm{GN}=\mathrm{HBB}$

$P E=3 \quad S V=1$

D9YZU5_HUMAN (+1)

$\begin{array}{llll}16 & 0.079 & 0.411 & 0.880\end{array}$

0.393

$0.377 \quad 0.000$

0.357

0.311

$\mathrm{PE}=2 \mathrm{SV}=1$ (2)d protein $\mathrm{OS}=$ Homo sapiens

Uncharacterized protein $\mathrm{OS}=$ Homo sapiens

$P E=2 S V=1$

B4E1Z4_HUMAN

$\begin{array}{llllllll}141 & 0.333 & 0.350 & 0.375 & 0.309 & 0.293 & 0.471 & 0.355\end{array}$

0.064

Beta-2-glycoprotein I (Fragment) OS=Homo

Q8NEJ1_HUMAN

$\begin{array}{llll}25 & 1.031 & 0.000 & 0.000\end{array}$

0.000

$0.000 \quad 1.077$

0.351

0.544

D9IWP9_HUMAN

$\begin{array}{llllllll}36 & 0.293 & 0.313 & 0.281 & 0.215 & 0.271 & 0.641 & 0.336\end{array}$

0.153

Anti-FactorVIII scFv (Fragment) OS=Homo

sapiens $\mathrm{PE}=2 \mathrm{SV}=1$

A2KBC6_HUMAN

$\begin{array}{llll}25 & 0.355 & 0.376 & 0.370\end{array}$

0.387

0.35

Transthyretin $\mathrm{OS}=$ Homo sapiens $\mathrm{PE}=2 \mathrm{SV}=1$

A6XGL1_HUMAN (+2)

20

$\begin{array}{lll}0.169 & 0.188 & 0.374\end{array}$

0.556

$0.348 \quad 0.330$

0.329

0.097

Fibronectin 1 , isoform CRA $n$ OS $=$ Homo

sapiens $\mathrm{GN}=\mathrm{FN} 1 \mathrm{PE}=4 \mathrm{SV}=1$

cDNA FLJ56954, highly similar to Inter-alpha-

trypsin inhibitor heavy chain $\mathrm{H} 1 \mathrm{OS}=\mathrm{Homo}$

75 sapiens $P E=2 \mathrm{SV}=1$

C4b-binding protein alpha chain $\mathrm{OS}=\mathrm{HomO}$

sapiens $\mathrm{GN}=\mathrm{C} 4 \mathrm{BPA} P E=1 \mathrm{SV}=2$

MS-D4 heavy chain variable region (Fragment)

$77 \quad O S=$ Homo sapiens $P E=2 \mathrm{SV}=1$

GCT-A10 heavy chain variable region

A0A024R462_HUMAN

$\begin{array}{llll}259 & 0.334 & 0.181 & 0.36\end{array}$

0.226

0.398

0.327

0.141

(Fragment) OS=Homo sapiens PE=2 SV=1

Serpin peptidase inhibitor, clade $C$

(Antithrombin), member 1 , isoform CRA a

B7Z539_HUMAN
C4BPA_HUMAN

$\begin{array}{lllllllll}72 & 0.510 & 0.000 & 0.520 & 0.000 & 0.715 & 0.000 & 0.291 & 0.327\end{array}$

79 OS=Homo sapiens $\mathrm{GN}=\mathrm{SERPINC} 1 \mathrm{PE}=3 \mathrm{SV}=1$ CDNA FLJ53025, highly similar to Complement

C4-B OS=Homo sapiens $P E=2 \mathrm{SV}=1$

Protein IGHV3-74 (Fragment) OS=Homo

sapiens $\mathrm{GN}=$ IGHV3-74 PE $=1 \mathrm{SV}=1$

Protein SAA2-SAA4 OS=Homo sapiens

$82 \mathrm{GN}=\mathrm{SAA} 2-\mathrm{SAA} 4 \mathrm{PE}=4 \mathrm{SV}=1$

IGL@ protein OS=Homo sapiens GN=IGL@

$P E=1 S V=1$

$\begin{array}{llll}67 & 0.202 & 0.294 & 0.151\end{array}$

AOAOX9UWK7_HUMAN

$\begin{array}{llll}14 & 0.332 & 0.268 & 0.346\end{array}$

0.180

$0.201 \quad 0.689$

0.286

0.203

A0A120HG46_HUMAN

$\begin{array}{llll}14 & 0.392 & 0.000 & 0.566\end{array}$

0.242

0.331

84 A30 (Fragment) $\mathrm{OS}=$ Homo sapiens $\mathrm{PE}=4 \mathrm{SV}=1$

Protein AMBP OS=Homo sapiens GN=AMBP

$P E=1 S V=1$

Ig lambda- 7 chain $\mathrm{C}$ region OS=Homo sapiens

$G N=I G L C 7 P E=1 \quad S V=2$

Complement $\mathrm{C} 5 \mathrm{OS}=$ Homo sapiens $\mathrm{GN}=\mathrm{C} 5$

$87 \quad \mathrm{PE}=1 \mathrm{SV}=4$

Apolipoprotein A-II OS=Homo sapiens

$88 \mathrm{GN}=\mathrm{APOA} 2 \mathrm{PE}=1 \mathrm{SV}=1$

Gelsolin OS=Homo sapiens $G N=G S N P E=1$

$\mathrm{SV}=1$

CDNA FLJ55606, highly similar to Alpha-2-HS-

90 glycoprotein OS=Homo sapiens $P E=2 \mathrm{SV}=1$

Insulin-like growth factor-binding protein

complex acid labile subunit OS=Homo sapiens

$91 \mathrm{GN}=$ IGFALS PE=1 SV=1

Histidine-rich glycoprotein OS=Homo sapiens

$92 \mathrm{GN}=\mathrm{HRG} \quad \mathrm{PE}=1 \mathrm{SV}=1$

Actin, cytoplasmic 1 OS=Homo sapiens

$93 \mathrm{GN}=\mathrm{ACTB} \quad \mathrm{PE}=1 \mathrm{SV}=1$

Ig heavy chain V-III region GAL OS=Homo

94 sapiens $\mathrm{PE}=1 \mathrm{SV}=1$

Complement factor $\mathrm{H}$-related protein 1

95 OS=Homo sapiens GN=CFHR1 PE=1 SV=2

Complement component 9 , isoform CRA a

96 OS=Homo sapiens $\mathrm{GN}=\mathrm{C} 9 \mathrm{PE}=4 \mathrm{SV}=1$

$\mathrm{N}$-acetylmuramoyl-L-alanine amidase OS=Homo

97 sapiens $\mathrm{GN}=P G L Y R P 2 \mathrm{PE}=1 \mathrm{SV}=1$

cDNA, FLJ94213, highly similar to Homo

sapiens pregnancy-zone protein (PZP), mRNA

98 OS=Homo sapiens $P E=2$ SV $=1$

GCT-A4 light chain variable region (Fragment)

$99 \quad \mathrm{OS}=$ Homo sapiens $\mathrm{PE}=2 \mathrm{SV}=1$

Apolipoprotein A-IV OS=Homo sapiens

$100 \quad G N=A P O A 4 P E=1 S V=3$

Inter-alpha-trypsin inhibitor heavy chain $\mathrm{H} 3$

101 OS=Homo sapiens $G N=I T \mid H 3 \quad P E=1 \quad S V=2$

A0A024R944_HUMAN (+1)

$53 \quad 0.255$

B7Z1F8_HUMAN

30

$\begin{array}{llll}0.255 & 0.239 & 0.241 & 0.283\end{array}$

AOA0B4J1X5_HUMAN

$\begin{array}{llll}30 & 0.000 & 1.534 & 0.000\end{array}$

0.000

$\begin{array}{ll}0.280 & 0.259\end{array}$

0.260

$13 \quad 0.3$

A0A096LPE2_HUMAN

$\begin{array}{llll}23 & 0.275 & 0.286 & 0.306\end{array}$

0.223

$0.000 \quad 0.000$

0.256

0.626

Q8N5F4_HUMAN

$25 \quad 0.000$

0.294

0.101

A2MYE1_HUMAN (+1)

$\begin{array}{lllll}10 & 0.211 & 0.470 & 0.220 & 0.290\end{array}$

0.000

0.101

0.000

0.255

0.137

AMBP_HUMAN

39

$\begin{array}{llll}0.217 & 0.229 & 0.271 & 0.161\end{array}$

$\begin{array}{ll}0.000 & 0.000 \\ 0.186 & 0.110\end{array}$

LAC7_HUMAN

$\begin{array}{llll}11 & 0.000 & 1.452 & 0.000\end{array}$

0.161

$0.214 \quad 0.394$

0.254

0.077

CO5 HUMAN

$\begin{array}{llll}188 & 0.234 & 0.270 & 0.255\end{array}$

0.201

$0.000 \quad 0.000$

$0.234 \quad 0.240$

APOA2_HUMAN (+1)

$\begin{array}{llll}11 & 0.269 & 0.213 & 0.240\end{array}$

0.308

0.379

GELS_HUMAN

$86 \quad 0.18$

$0.137 \quad 0.184$

0.292

$0.379 \quad 0.000$

B7Z8Q2_HUMAN (+2)

$\begin{array}{llll}47 & 0.144 & 0.220 & 0.178\end{array}$

0.267

0.243

0.275

0.235

0.235

$\begin{array}{lll}0.178 & 0.304 & 0.215\end{array}$

0.215

0.061

ALS_HUMAN $(+1)$

$\begin{array}{lllllllll}66 & 0.198 & 0.178 & 0.253 & 0.212 & 0.232 & 0.208 & 0.214 & 0.026\end{array}$

HRG_HUMAN

$\begin{array}{llllllll}60 & 0.000 & 0.415 & 0.382 & 0.000 & 0.000 & 0.467 & 0.211\end{array}$ 0.232

ACTB_HUMAN (+2)

$\begin{array}{lllll}42 & 0.272 & 0.179 & 0.241 & 0.368\end{array}$

$0.077 \quad 0.105$

HV320 HUMAN

$\begin{array}{llll}13 & 0.000 & 0.433 & 0.000\end{array}$

0.409

$0.392 \quad 0.000$

0.207

0.109

FHR1_HUMAN

$\begin{array}{lllll}38 & 0.289 & 0.210 & 0.324\end{array}$

0.000

0.000

A0A024R035_HUMAN (+1)

63

PGRP2_HUMAN

$\begin{array}{llll}63 & 0.221 & 0.268 & 0.189\end{array}$

0.161

$\begin{array}{llll}62 & 0.191 & 0.212 & 0.149\end{array}$

0.203

0.11

0.000

0.405

0.206

0.226

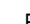

B2R950_HUMAN (+1)

\begin{tabular}{llll}
164 & 0.188 & 0.235 & 0.156 \\
\hline
\end{tabular}

0.139

$0.180 \quad 0.23$

$0.218 \quad 0.196$

A0A0X9T7V9_HUMAN

\begin{tabular}{|c|c|c|c|c|c|c|c|c|}
\hline 98 & $\mathrm{OS}=$ Homo sapiens $\mathrm{PE}=2 \mathrm{SV}=1$ & B2R950_HUMAN (+1) & 164 & 0.188 & 0.235 & 0.156 & 0.139 & 0.257 \\
\hline 99 & $\begin{array}{l}\text { GCT-A4 light chain variable region (Fragment) } \\
\text { OS=Homo sapiens } P E=2 S V=1\end{array}$ & A0A0X9T7V9_HUMAN & 12 & 0.176 & 0.157 & 0.147 & 0.201 & 0.193 \\
\hline 100 & $\begin{array}{l}\text { Apolipoprotein A-IV OS=Homo sapiens } \\
\text { GN=APOA4 PE }=1 \mathrm{SV}=3\end{array}$ & APOA4_HUMAN & 45 & 0.094 & 0.115 & 0.205 & 0.247 & 0.155 \\
\hline 101 & $\begin{array}{l}\text { Inter-alpha-trypsin inhibitor heavy chain H3 } \\
\text { OS=Homo sapiens } \mathrm{GN}=\mathrm{ITIH} 3 \mathrm{PE}=1 \mathrm{SV}=2\end{array}$ & ITIH3_HUMAN & 100 & 0.241 & 0.244 & 0.176 & 0.111 & 0.190 \\
\hline
\end{tabular}

APOA4_HUMAN

\begin{tabular}{|c|c|c|c|c|c|c|c|c|}
\hline 98 & $\mathrm{OS}=$ Homo sapiens $\mathrm{PE}=2 \mathrm{SV}=1$ & B2R950_HUMAN (+1) & 164 & 0.188 & 0.235 & 0.156 & 0.139 & 0.257 \\
\hline 99 & $\begin{array}{l}\text { GCT-A4 light chain variable region (Fragment) } \\
\text { OS=Homo sapiens } P E=2 S V=1\end{array}$ & A0A0X9T7V9_HUMAN & 12 & 0.176 & 0.157 & 0.147 & 0.201 & 0.193 \\
\hline 100 & $\begin{array}{l}\text { Apolipoprotein A-IV OS=Homo sapiens } \\
\text { GN=APOA4 PE }=1 \mathrm{SV}=3\end{array}$ & APOA4_HUMAN & 45 & 0.094 & 0.115 & 0.205 & 0.247 & 0.155 \\
\hline 101 & $\begin{array}{l}\text { Inter-alpha-trypsin inhibitor heavy chain H3 } \\
\text { OS=Homo sapiens } \mathrm{GN}=\mathrm{ITIH} 3 \mathrm{PE}=1 \mathrm{SV}=2\end{array}$ & ITIH3_HUMAN & 100 & 0.241 & 0.244 & 0.176 & 0.111 & 0.190 \\
\hline
\end{tabular}

\begin{tabular}{|c|c|c|c|c|c|c|c|c|}
\hline 98 & $\mathrm{OS}=$ Homo sapiens $\mathrm{PE}=2 \mathrm{SV}=1$ & B2R950_HUMAN (+1) & 164 & 0.188 & 0.235 & 0.156 & 0.139 & 0.257 \\
\hline 99 & $\begin{array}{l}\text { GCT-A4 light chain variable region (Fragment) } \\
\text { OS=Homo sapiens } P E=2 S V=1\end{array}$ & A0A0X9T7V9_HUMAN & 12 & 0.176 & 0.157 & 0.147 & 0.201 & 0.193 \\
\hline 100 & $\begin{array}{l}\text { Apolipoprotein A-IV OS=Homo sapiens } \\
\text { GN=APOA4 PE }=1 \mathrm{SV}=3\end{array}$ & APOA4_HUMAN & 45 & 0.094 & 0.115 & 0.205 & 0.247 & 0.155 \\
\hline 101 & $\begin{array}{l}\text { Inter-alpha-trypsin inhibitor heavy chain H3 } \\
\text { OS=Homo sapiens } \mathrm{GN}=\mathrm{ITIH} 3 \mathrm{PE}=1 \mathrm{SV}=2\end{array}$ & ITIH3_HUMAN & 100 & 0.241 & 0.244 & 0.176 & 0.111 & 0.190 \\
\hline
\end{tabular}

\begin{tabular}{|c|c|c|c|c|c|c|c|c|c|}
\hline 98 & $\begin{array}{l}\text { sapiens pregnancy-zone proteln }(P L P) \text {, mRNA } \\
\text { OS=Homo sapiens } \mathrm{PE}=2 \mathrm{SV}=1\end{array}$ & B2R950_HUMAN (+1) & 164 & 0.188 & 0.235 & 0.156 & 0.139 & 0.257 & 0.184 \\
\hline 99 & $\begin{array}{l}\text { GCT-A4 light chain variable region (Fragment) } \\
\text { OS=Homo sapiens } P E=2 S V=1\end{array}$ & A0A0X9T7V9_HUMAN & 12 & 0.176 & 0.157 & 0.147 & 0.201 & 0.193 & 0.229 \\
\hline 100 & $\begin{array}{l}\text { Apolipoprotein A-IV OS=Homo sapiens } \\
\text { GN=APOA4 PE=1 SV=3 }\end{array}$ & APOA4_HUMAN & 45 & 0.094 & 0.115 & 0.205 & 0.247 & 0.155 & 0.244 \\
\hline 101 & $\begin{array}{l}\text { Inter-alpha-trypsin inhibitor heavy chain } \mathrm{H} 3 \\
\mathrm{OS}=\text { Homo sapiens } \mathrm{GN}=\mathrm{ITIH} 3 \mathrm{PE}=1 \mathrm{SV}=2\end{array}$ & ITIH3_HUMAN & 100 & 0.241 & 0.244 & 0.176 & 0.111 & 0.190 & 0.093 \\
\hline
\end{tabular}

100

\begin{tabular}{|c|c|c|c|c|c|c|c|c|}
\hline 98 & $\mathrm{OS}=$ Homo sapiens $\mathrm{PE}=2 \mathrm{SV}=1$ & B2R950_HUMAN (+1) & 164 & 0.188 & 0.235 & 0.156 & 0.139 & 0.257 \\
\hline 99 & $\begin{array}{l}\text { GCT-A4 light chain variable region (Fragment) } \\
\text { OS=Homo sapiens } P E=2 \mathrm{SV}=1\end{array}$ & A0A0X9T7V9_HUMAN & 12 & 0.176 & 0.157 & 0.147 & 0.201 & 0.193 \\
\hline 100 & $\begin{array}{l}\text { Apolipoprotein A-IV OS=Homo sapiens } \\
\text { GN=APOA4 PE=1 SV=3 }\end{array}$ & APOA4_HUMAN & 45 & 0.094 & 0.115 & 0.205 & 0.247 & 0.155 \\
\hline 101 & $\begin{array}{l}\text { Inter-alpha-trypsin inhibitor heavy chain } \mathrm{H} 3 \\
\mathrm{OS}=\text { Homo sapiens } \mathrm{GN}=\mathrm{ITIH} 3 \mathrm{PE}=1 \mathrm{SV}=2\end{array}$ & ITIH3_HUMAN & 100 & 0.241 & 0.244 & 0.176 & 0.111 & 0.190 \\
\hline
\end{tabular}

$0.241 \quad 0.24$

0.176

0.111 
102 OS=Homo sapiens $\mathrm{PE}=2 \mathrm{SV}=1$

Apolipoprotein E OS=Homo sapiens $\mathrm{GN}=\mathrm{APOE}$

$103 \quad P E=1 S V=1$

Uncharacterized protein OS=Homo sapiens

$104 \quad P E=2 S V=1$

Apolipoprotein D OS=Homo sapiens GN=APOD

$105 \quad P E=1$ SV=1

Zinc-alpha-2-glycoprotein OS=Homo sapiens

106 GN=AZGP1 PE=1 SV=2

IGL@ protein OS=Homo sapiens GN=IGL@

$107 \quad \mathrm{PE}=2 \mathrm{SV}=1$

Immunglobulin heavy chain variable region

108 (Fragment) OS=Homo sapiens $\mathrm{PE}=4 \mathrm{SV}=1$

Coagulation factor XII OS=Homo sapiens

$109 \quad \mathrm{GN}=\mathrm{F} 12 \mathrm{PE}=1 \mathrm{SV}=3$

Alpha-2-antiplasmin OS=Homo sapiens

$110 \mathrm{GN}=\mathrm{SERPINF} 2 \mathrm{PE}=1 \mathrm{SV}=3$

GCT-A5 light chain variable region (Fragment)

111 OS=Homo sapiens $\mathrm{PE}=2 \mathrm{SV}=1$

Complement component $\mathrm{C} 6$ OS=Homo sapiens

$112 \mathrm{GN}=\mathrm{C} 6 \mathrm{PE}=1 \mathrm{SV}=3$

Serum amyloid P-component OS=Homo

113 sapiens $\mathrm{GN}=\mathrm{APCS} \mathrm{PE}=1 \mathrm{SV}=2$

Complement component $\mathrm{C} 8$ gamma chain

114 OS=Homo sapiens $\mathrm{GN}=\mathrm{C} 8 \mathrm{G} \quad \mathrm{PE}=1 \mathrm{SV}=3$

Alpha-1-acid glycoprotein 2 OS=Homo sapiens

115 GN=ORM2 $P E=1 \mathrm{SV}=2$

Serum paraoxonase/arylesterase $1 \mathrm{OS}=\mathrm{Homo}$

116

sapiens $\mathrm{GN}=\mathrm{PON} 1 \mathrm{PE}=1 \mathrm{SV}=3$

26

Complement component 1 , q subcomponent, $B$

chain, isoform CRA_a OS=Homo sapiens

$117 \mathrm{GN}=\mathrm{C} 1 \mathrm{QB} \quad \mathrm{PE}=4 \mathrm{SV}=1$

Uncharacterized protein OS=Homo sapiens

$118 \mathrm{PE}=1 \mathrm{SV}=1$

Retinol binding protein 4, plasma, isoform

CRA_b OS=Homo sapiens $\mathrm{GN}=\mathrm{RBP} 4 \mathrm{PE}=1$

$119 \quad \mathrm{SV}=2$

IGL@ protein OS=Homo sapiens GN=IGL@

$120 \quad P E=1 S V=1$

Complement component 1, q subcomponent, C

chain, isoform CRA_a OS=Homo sapiens

$121 \quad G N=C 1 Q C P E=4 \quad S V=1$

HCG40889, isoform CRA_b OS=Homo sapiens

$122 \mathrm{GN}=h \mathrm{hG}_{2} 40889 \mathrm{PE}=4 \mathrm{SV}=1$

GCT-A5 heavy chain variable region (Fragment)

123 OS=Homo sapiens $\mathrm{PE}=2 \mathrm{SV}=1$

Complement component $\mathrm{C} 8$ alpha chain

124 OS=Homo sapiens $\mathrm{GN}=\mathrm{C} 8 \mathrm{~A} \mathrm{PE}=1 \mathrm{SV}=2$

Vitamin K-dependent protein S OS=Homo

125 sapiens $\mathrm{GN}=\mathrm{PROS} 1 \mathrm{PE}=1 \mathrm{SV}=1$

Afamin OS=Homo sapiens GN=AFM PE $=1$

$126 \quad S V=1$

Plasma kallikrein heavy chain (Fragment)

127 OS=Homo sapiens $\mathrm{GN}=\mathrm{KLKB} 1 \mathrm{PE}=3 \mathrm{SV}=1$

Complement C1r subcomponent OS=Homo

sapiens $\mathrm{GN}=\mathrm{C} 1 \mathrm{R} \mathrm{PE}=2 \mathrm{SV}=1$

CD5 antigen-like OS=Homo sapiens $\mathrm{GN}=\mathrm{CD} 5 \mathrm{~L}$

$129 \quad \mathrm{PE}=1 \mathrm{SV}=1$

GCT-A8 heavy chain variable region (Fragment)

130 OS=Homo sapiens $P E=2 \mathrm{SV}=1$

131 Complement C1s subcomponent OS=Homo

sapiens $\mathrm{GN}=\mathrm{C} 1 \mathrm{~S} P \mathrm{PE}=1 \mathrm{SV}=1$

Complement component 8, beta polypeptide,

isoform CRA_b OS=Homo sapiens $\mathrm{GN}=\mathrm{C} 8 \mathrm{~B}$

$132 \quad P E=2 S V=1$

CDNA FLJ78207, highly similar to Human

complement protein component C7 mRNA

133 OS=Homo sapiens $P E=2$ SV=1

Ig heavy chain $V$-III region WEA OS=Homo

134 sapiens $\mathrm{PE}=1 \mathrm{SV}=1$

Carboxypeptidase B2 OS=Homo sapiens

$135 \mathrm{GN}=\mathrm{CPB} 2 \mathrm{PE}=4 \mathrm{SV}=1$

130 Lumican OS=Homo sapiens $\mathrm{GN}=\mathrm{LUM} P E=1$

$136 \quad \mathrm{SV}=2$

$\mathrm{SV}=2$
Vitamin D-binding protein OS=Homo sapiens

$137 \mathrm{GN}=\mathrm{GC} P E=1 \mathrm{SV}=1$

\begin{tabular}{|c|c|c|c|c|c|c|c|c|c|}
\hline A0A0X9TD47_HUMAN & 12 & 0.000 & 0.235 & 0.220 & 0.322 & 0.271 & 0.000 & 0.175 & 0.140 \\
\hline APOE_HUMAN (+1) & 36 & 0.141 & 0.183 & 0.159 & 0.188 & 0.167 & 0.198 & 0.173 & 0.021 \\
\hline A0A5E4_HUMAN & 25 & 1.031 & 0.000 & 0.000 & 0.000 & 0.000 & 0.000 & 0.172 & 0.421 \\
\hline APOD_HUMAN & 21 & 0.161 & 0.112 & 0.147 & 0.184 & 0.177 & 0.235 & 0.169 & 0.041 \\
\hline ZA2G_HUMAN & 34 & 0.174 & 0.152 & 0.207 & 0.185 & 0.082 & 0.210 & 0.168 & 0.048 \\
\hline Q567P1_HUMAN & 25 & 0.997 & 0.000 & 0.000 & 0.000 & 0.000 & 0.000 & 0.166 & 0.407 \\
\hline Q0ZCH6_HUMAN & 14 & 0.211 & 0.134 & 0.126 & 0.242 & 0.166 & 0.118 & 0.166 & 0.050 \\
\hline FA12_HUMAN (+1) & 68 & 0.155 & 0.138 & 0.129 & 0.142 & 0.157 & 0.259 & 0.163 & 0.048 \\
\hline A2AP_HUMAN & 55 & 0.154 & 0.154 & 0.160 & 0.149 & 0.143 & 0.210 & 0.162 & 0.024 \\
\hline AOAOX9UWL5_HUMAN & 12 & 0.317 & 0.352 & 0.293 & 0.000 & 0.000 & 0.000 & 0.160 & 0.177 \\
\hline CO6_HUMAN & 105 & 0.177 & 0.165 & 0.134 & 0.115 & 0.168 & 0.194 & 0.159 & 0.029 \\
\hline SAMP_HUMAN (+1) & 25 & 0.203 & 0.188 & 0.141 & 0.116 & 0.130 & 0.176 & 0.159 & 0.035 \\
\hline CO8G_HUMAN & 22 & 0.211 & 0.171 & 0.160 & 0.176 & 0.042 & 0.175 & 0.156 & 0.058 \\
\hline A1AG2_HUMAN & 24 & 0.141 & 0.196 & 0.220 & 0.101 & 0.116 & 0.137 & 0.152 & 0.047 \\
\hline PON1_HUMAN & 40 & 0.106 & 0.141 & 0.121 & 0.169 & 0.139 & 0.233 & 0.152 & 0.045 \\
\hline A0A024RAB9_HUMAN (+3) & 27 & 0.125 & 0.157 & 0.114 & 0.161 & 0.120 & 0.224 & 0.150 & 0.041 \\
\hline Q8TCD0_HUMAN & 26 & 0.000 & 0.000 & 0.000 & 0.874 & 0.000 & 0.000 & 0.146 & 0.357 \\
\hline Q5VY30_HUMAN (+2) & 23 & 0.129 & 0.061 & 0.153 & 0.168 & 0.121 & 0.239 & 0.145 & 0.059 \\
\hline Q5FWF9_HUMAN & 25 & 0.000 & 0.000 & 0.863 & 0.000 & 0.000 & 0.000 & 0.144 & 0.352 \\
\hline A0A024RAA7_HUMAN (+1) & 26 & 0.114 & 0.163 & 0.169 & 0.112 & 0.089 & 0.190 & 0.139 & 0.040 \\
\hline A0A024R962_HUMAN & 139 & 0.000 & 0.000 & 0.000 & 0.000 & 0.000 & 0.834 & 0.139 & 0.340 \\
\hline AOAOX9TOH6_HUMAN & 12 & 0.458 & 0.000 & 0.367 & 0.000 & 0.000 & 0.000 & 0.137 & 0.215 \\
\hline CO8A_HUMAN & 65 & 0.175 & 0.152 & 0.135 & 0.112 & 0.121 & 0.127 & 0.137 & 0.023 \\
\hline PROS_HUMAN (+2) & 75 & 0.141 & 0.175 & 0.088 & 0.084 & 0.179 & 0.139 & 0.134 & 0.041 \\
\hline AFAM_HUMAN & 69 & 0.073 & 0.102 & 0.057 & 0.112 & 0.188 & 0.271 & 0.134 & 0.081 \\
\hline HOYAC1_HUMAN & 77 & 0.082 & 0.098 & 0.120 & 0.113 & 0.157 & 0.221 & 0.132 & 0.050 \\
\hline B4DPQ0_HUMAN & 82 & 0.113 & 0.155 & 0.113 & 0.112 & 0.102 & 0.181 & 0.129 & 0.031 \\
\hline CD5L_HUMAN & 38 & 0.122 & 0.111 & 0.127 & 0.089 & 0.122 & 0.202 & 0.129 & 0.038 \\
\hline A0A0X9V9C4_HUMAN & 13 & 0.227 & 0.000 & 0.237 & 0.000 & 0.000 & 0.296 & 0.127 & 0.141 \\
\hline C1S_HUMAN & 77 & 0.104 & 0.140 & 0.103 & 0.107 & 0.133 & 0.164 & 0.125 & 0.025 \\
\hline B7Z550_HUMAN (+4) & 60 & 0.120 & 0.110 & 0.125 & 0.105 & 0.100 & 0.165 & 0.121 & 0.023 \\
\hline A8K2T4_HUMAN (+1) & 93 & 0.141 & 0.146 & 0.099 & 0.068 & 0.080 & 0.189 & 0.121 & 0.046 \\
\hline HV302_HUMAN & 12 & 0.211 & 0.000 & 0.293 & 0.201 & 0.000 & 0.000 & 0.118 & 0.133 \\
\hline A0A087WSY5_HUMAN (+1) & 44 & 0.086 & 0.128 & 0.100 & 0.143 & 0.116 & 0.125 & 0.116 & 0.020 \\
\hline LUM_HUMAN (+1) & 38 & 0.100 & 0.124 & 0.116 & 0.127 & 0.098 & 0.116 & 0.113 & 0.012 \\
\hline D6RF35_HUMAN & 53 & 0.000 & 0.000 & 0.665 & 0.000 & 0.000 & 0.000 & 0.111 & 0.271 \\
\hline
\end{tabular}

61 
$\lg \mathrm{G} \mathrm{H}$ chain $\mathrm{OS}=$ Homo sapiens $\mathrm{PE}=2 \mathrm{SV}=1$

Keratin, type II cytoskeletal 2 epidermal

155 OS=Homo sapiens GN=KRT2 PE=1 SV=2 APOL1 protein (Fragment) OS=Homo sapiens

$156 \mathrm{GN}=\mathrm{APOL} 1 \mathrm{PE}=2 \mathrm{SV}=1$

Angiotensinogen variant (Fragment) OS=Homo

Protein IGHV3-73 (Fragment) OS=Homo

158 sapiens $G N=I G H V 3-73 \quad P E=1 \quad S V=1$

Rheumatoid factor $\mathrm{C} 6$ light chain (Fragment)

159 OS=Homo sapiens $\mathrm{GN}=\mathrm{V}<\mathrm{kappa}>1 \mathrm{PE}=2 \mathrm{SV}=1$ Thyroxine-binding globulin OS=Homo sapiens

160 GN=SERPINA7 PE=1 SV=2

Carboxypeptidase $\mathrm{N}$ subunit $2 \mathrm{OS}=\mathrm{Homo}$

161 sapiens $\mathrm{GN}=\mathrm{CPN} 2 \mathrm{PE}=1 \mathrm{SV}=3$

Ficolin-3 OS=Homo sapiens $\mathrm{GN}=\mathrm{FCN} 3 \mathrm{PE}=1$

$162 \quad S V=2$

Complement factor I OS=Homo sapiens

$163 \mathrm{GN}=\mathrm{CFI} P E=1 \mathrm{SV}=2$

164 IgG L chain OS=Homo sapiens $P E=2$ SV=1 Carboxypeptidase $\mathrm{N}$ catalytic chain $\mathrm{OS}=\mathrm{Homo}$ 165 sapiens $\mathrm{GN}=\mathrm{CPN} 1 \mathrm{PE}=1 \mathrm{SV}=1$

VH6DJ protein (Fragment) OS=Homo sapiens

$166 \mathrm{GN}=V H 6 \mathrm{DJ} \mathrm{PE}=2 \mathrm{SV}=1$

Keratin, type I cytoskeletal 9 OS=Homo sapiens

$167 \mathrm{GN}=\mathrm{KRT}$ 9 $P E=1 \mathrm{SV}=3$

Coagulation factor $\mathrm{V} O \mathrm{OS}=\mathrm{Homo}$ sapiens $\mathrm{GN}=\mathrm{F5}$

$168 \quad \mathrm{PE}=4 \mathrm{SV}=1$

Ig heavy chain V-III region BUT OS=Homo

169 sapiens $P E=1 \mathrm{SV}=1$

Galectin-3-binding protein OS=Homo sapiens

170 GN=LGALS3BP PE=1 SV=1

V1-2 protein (Fragment) OS=Homo sapiens

$171 \mathrm{GN}=\mathrm{V} 1-2 \mathrm{PE}=4 \mathrm{SV}=1$

Serpin peptidase inhibitor, clade A (Alpha-1

antiproteinase, antitrypsin), member 4 , isoform

CRA $\_$a OS=Homo sapiens $\mathrm{GN}=$ SERPINA4

$172 \quad P E=3 \quad S V=1$

V1-22 protein (Fragment) OS=Homo sapiens

$173 \mathrm{GN}=\mathrm{V} 1-22 \mathrm{PE}=1 \mathrm{SV}=1$

Thrombospondin- 1 OS=Homo sapiens

$174 \mathrm{GN}=\mathrm{THBS1} \mathrm{PE}=1 \mathrm{SV}=2$

AON5G3_HUMAN $\begin{array}{llll}13 & 0.000 & 0.000 & 0.643\end{array}$ 0.000 0.000 0.000 0.107 $\begin{array}{llll}13 & 0.325 & 0.000 & 0.169\end{array}$

0.149
$0.000 \quad 0.000$ A3KPE2_HUMAN (+2) $\begin{array}{llll}11 & 0.077 & 0.085 & 0.120\end{array}$ 0.176 $0.084 \quad 0.100$ 0.107 $\begin{array}{llllllllll}\text { A0A075B6K3_HUMAN (+1) } & 13 & 0.260 & 0.000 & 0.373 & 0.000 & 0.000 & 0.000 & 0.105 & 0.167\end{array}$ AOAOAOMS11 HUMAN 13 $\begin{array}{lll}0.227 & 0.217 & 0.000\end{array}$ 0.186 $0.000 \quad 0.000$ 0.105 0.116 H6VRF8_HUMAN (+3) 66 $0.141 \quad 0.107 \quad 0.093$ 0.059 $0.176 \quad 0.042$ 0.103 0.050 B1N7B8_HUMAN

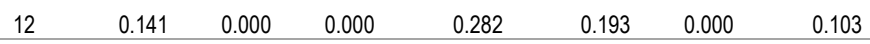
0.121

CLUS_HUMAN $\begin{array}{llllllll}52 & 0.097 & 0.099 & 0.110 & 0.074 & 0.089 & 0.116 & 0.098\end{array}$ $\begin{array}{llllllllll}\text { A0A075B6R9_HUMAN (+1) } & 13 & 0.065 & 0.072 & 0.000 & 0.149 & 0.214 & 0.085 & 0.097 & 0.074\end{array}$ Q68CK4_HUMAN

$38 \quad 0.111$

A0A068LKQ2_HUMAN

PEDF_HUMAN

$\begin{array}{llll}13 & 0.000 & 0.144 & 0.135\end{array}$
0.115

0.08

IGJ HUMAN 46 $\begin{array}{lll}0.092 & 0.061 & 0.096\end{array}$ 0.074

$0.107-0.085$
0.097 0.022

K1C10_HUMAN $\begin{array}{lllllllll}18 & 0.047 & 0.000 & 0.098 & 0.161 & 0.103 & 0.122 & 0.089 & 0.057\end{array}$ CO2 HUMAN $(+3)$ $\begin{array}{llll}59 & 0.129 & 0.111 & 0.082\end{array}$

A0A125UOV2_HUMAN 83 0.00

S6B2B6_HUMAN

$\begin{array}{llllllll}14 & 0.000 & 0.000 & 0.000 & 0.518 & 0.000 & 0.000 & 0.086\end{array}$

0.087

K22E_HUMAN

$\begin{array}{llll}29 & 0.000 & 0.000 & 0.516\end{array}$

0.000

$0.000 \quad 0.000$

0.086

$\begin{array}{llllllll}65 & 0.097 & 0.116 & 0.075 & 0.037 & 0.143 & 0.000 & 0.078\end{array}$

0.052

A5PL32 HUMAN (+2)

$\begin{array}{llllllll}49 & 0.078 & 0.077 & 0.045 & 0.109 & 0.114 & 0.045 & 0.078\end{array}$

Q53GY3_HUMAN (+3)

$\begin{array}{lllll}53 & 0.064 & 0.044 & 0.042 & 0.073\end{array}$

$0.096 \quad 0.104$

0.070

0.030

A0A0B4J1V6_HUMAN

$\begin{array}{llll}9 & 0.000 & 0.000 & 0.000\end{array}$

0.000

$0.412 \quad 0.000$

0.069

0.026

A0N5G1_HUMAN

$\begin{array}{llll}13 & 0.195 & 0.108 & 0.000\end{array}$

0.000

$0.107 \quad 0.000$

0.068

THBG_HUMAN

$\begin{array}{llll}46 & 0.073 & 0.092 & 0.048\end{array}$

0.084

$0.071 \quad 0.036$

0.067

$\begin{array}{llllllllll}\text { CPN2_HUMAN } & 61 & 0.062 & 0.069 & 0.043 & 0.048 & 0.084 & 0.081 & 0.065 & 0.017 \\ \text { FCN3_HUMAN } & 33 & 0.077 & 0.057 & 0.053 & 0.073 & 0.056 & 0.067 & 0.064 & 0.010\end{array}$

$\begin{array}{llllllllll}\text { CPN2_HUMAN } & 61 & 0.062 & 0.069 & 0.043 & 0.048 & 0.084 & 0.081 & 0.065 & 0.017 \\ \text { FCN3_HUMAN } & 33 & 0.077 & 0.057 & 0.053 & 0.073 & 0.056 & 0.067 & 0.064 & 0.010\end{array}$

$\begin{array}{lccccccccc}\text { FCN3_HUMAN } & 33 & 0.077 & 0.057 & 0.053 & 0.073 & 0.056 & 0.067 & 0.064 & 0.010 \\ \text { CFAI_HUMAN (+2) } & 66 & 0.032 & 0.064 & 0.047 & 0.029 & 0.056 & 0.142 & 0.062 & 0.041\end{array}$

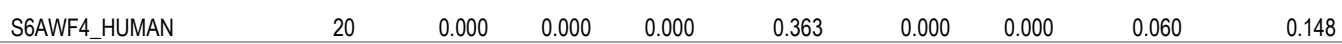

$\begin{array}{llllllllll}\text { CBPN_HUMAN } & 52 & 0.049 & 0.081 & 0.051 & 0.074 & 0.054 & 0.053 & 0.060 & 0.014\end{array}$

$\begin{array}{llllllllll}\text { A2N0T1_HUMAN } & 13 & 0.000 & 0.217 & 0.000 & 0.000 & 0.143 & 0.000 & 0.060 & 0.096\end{array}$

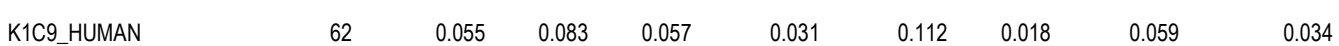

$\begin{array}{llllllllll}\text { AOAOAOMRJ7_HUMAN (+1) } & 252 & 0.064 & 0.071 & 0.054 & 0.046 & 0.070 & 0.048 & 0.059 & 0.011\end{array}$

HV306_HUMAN

LG3BP_HUMAN (+1)

$\begin{array}{llll}12 & 0.000 & 0.000 & 0.110\end{array}$

0.242

$\begin{array}{llll}65 & 0.117 & 0.072 & 0.041\end{array}$

0.022

$\begin{array}{lll}0.000 & 0.000 & 0.059\end{array}$
0.100

A2MYD6_HUMAN

$\begin{array}{llll}10 & 0.338 & 0.000 & 0.000\end{array}$

0.000

$0.000 \quad 0.000$

0.056

0.034

A0A024R619_HUMAN (+2)

$\begin{array}{llll}49 & 0.043 & 0.067 & 0.036\end{array}$

0.079

$0.076 \quad 0.034$

0.056

0.021

Q5NV88_HUMAN

$\begin{array}{llll}11 & 0.000 & 0.000 & 0.000\end{array}$

0.176

$0.000 \quad 0.150$

0.054

0.084

TSP1_HUMAN

129

$\begin{array}{lll}0.039 & 0.062 & 0.065\end{array}$

$\begin{array}{llll}0.075 & 0.054 & 0.021 & 0.053\end{array}$

0.019 
MS-F1 light chain variable region (Fragment)

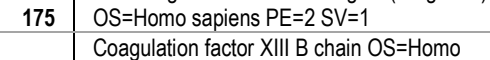

A0A0X9V9B3 HUMAN

$\begin{array}{lllllllll}12 & 0.000 & 0.078 & 0.073 & 0.081 & 0.077 & 0.000 & 0.052 & 0.040\end{array}$

176 sapiens $\mathrm{GN}=F 13 B \mathrm{BE}=1 \mathrm{SV}=3$

F13B_HUMAN

$\begin{array}{llll}76 & 0.039 & 0.025 & 0.064\end{array}$

0.032

$0.055 \quad 0.094$

0.051

0.025

$177 \mathrm{GN}=\mathrm{V} 1-13 \mathrm{PE}=4 \mathrm{SV}=1$

Talin-1 OS=Homo sapiens $G N=T L N 1 P E=1$

Q5NV69_HUMAN

$\begin{array}{llll}10 & 0.211 & 0.000 & 0.000\end{array}$

0.000

$0.093 \quad 0.000$

0.051

0.087

$178 \quad S V=3$

Lipoprotein, Lp(A) OS=Homo sapiens GN=LPA

$179 \quad P E=1 S V=1$

TLN1_HUMAN

$\begin{array}{llllllll}270 & 0.095 & 0.031 & 0.070 & 0.088 & 0.005 & 0.010 & 0.050\end{array}$ 0.040

Lipopolysaccharide-binding protein OS=Homo

180 sapiens $G N=L B P P E=1 S V=3$

Myosin-reactive immunoglobulin heavy chain

Q1HP67_HUMAN

227

$\begin{array}{lll}0.095 & 0.031 & 0.070 \\ 0.000 & 0.112 & 0.138\end{array}$

LBP_HUMAN (+1)

$\begin{array}{llll}53 & 0.040 & 0.062 & 0.058\end{array}$

0.000

0.01

$0.012 \quad 0.046$

0.062

variable region (Fragment) OS=Homo sapiens

$181 \quad \mathrm{PE}=2 \mathrm{SV}=1$

Complement factor $\mathrm{H}$-related protein 3

Q9UL89_HUMAN

$\begin{array}{llll}13 & 0.000 & 0.000 & 0.271\end{array}$

0.055

$\begin{array}{lll}0.026 & 0.031 & 0.045\end{array}$

0.015

SS-Homo sapiens $\mathrm{GN}=\mathrm{CFHR} 3 \mathrm{PE}=1 \mathrm{SV}=2$

sapiens serine (or cysteine) proteinase inhibitor

clade A(alpha-1 antiproteinase, antitrypsin),

member 6 (SERPINA6), mRNA OS=Homo

183 sapiens $P E=2 S V=1$

Carbonic anhydrase 1 OS=Homo sapiens

$184 \mathrm{GN}=\mathrm{CA} 1 \mathrm{PE}=1 \mathrm{SV}=2$

Hyaluronan-binding protein $2 \mathrm{OS}=$ Homo sapiens

$185 \mathrm{GN}=\mathrm{HABP} 2 \mathrm{PE}=1 \mathrm{SV}=1$

Protein IGHV5-51 (Fragment) OS=Homo

186 sapiens $G N=I G H V 5-51 \quad P E=1 \quad S V=1$

Serpin peptidase inhibitor, clade A (Alpha-1

antiproteinase, antitrypsin), member 10, isoform

CRA_b OS=Homo sapiens GN=SERPINA10

$187 \quad \mathrm{PE}=3 \mathrm{SV}=1$

FHR3 HUMAN

37

$\begin{array}{lll}0.148 & 0.000 & 0.000\end{array}$

0.000

0.000

0.00

.000

0.045

0.111

Glyceraldehyde-3-phosphate dehydrogenase

188 OS=Homo sapiens GN=GAPDH PE $=1$ SV $=3$

Anti-HER3 scFv (Fragment) OS=Homo sapiens

$\mathrm{PE}=2 \mathrm{SV}=1$

Phosphatidylinositol-glycan-specific

phospholipase D OS=Homo sapiens

$190 \mathrm{GN}=\mathrm{GPLD1} P E=1 \mathrm{SV}=3$

Coagulation factor XIII A chain OS=Homo

191 sapiens $G N=F 13 A 1 \quad P E=1 \quad S V=4$

Attractin OS=Homo sapiens GN=ATRN PE $=1$

$192 \quad S V=2$

Single-chain Fv (Fragment) OS=Homo sapiens

$193 \mathrm{GN}=\mathrm{scF} \vee \mathrm{PE}=1 \mathrm{SV}=1$

Myosin-reactive immunoglobulin light chain

variable region (Fragment) OS=Homo sapiens

194

$P E=1 S V=1$

195

CDNA FLJ60461, highly similar to Peroxiredoxin-

952 (EC 1.11.1.15) OS=Homo sapiens $P E=2 \mathrm{SV}=1$

Serpin peptidase inhibitor, clade A (Alpha-1

antiproteinase, antitrypsin), member 5 , isoform

CRA_a OS=Homo sapiens $G N=S E R P I N A 5$

$196 \quad P E=3 \mathrm{SV}=1$

$\begin{array}{llllllllll}\text { B2R9F2_HUMAN (+1) } & 45 & 0.019 & 0.083 & 0.020 & 0.043 & 0.052 & 0.037 & 0.042 & 0.024\end{array}$

CAH1_HUMAN (+1)

HABP2_HUMAN

$\begin{array}{ll}29 & 0.000\end{array}$

AOAOC4DH38_HUMAN

$\begin{array}{llll}63 & 0.034 & 0.030 & 0.014\end{array}$

0.033

0.00

$0.000 \quad 0.000$

$0.000 \quad 0.042$

0.060

$\begin{array}{llll}13 & 0.000 & 0.072 & 0.000\end{array}$

0.023

0.029

0.096

0.038

A0A024R6I6_HUMAN (+3)

G3P HUMAN (+1)

$\begin{array}{lllll}\text { A2J422_HUMAN } & 26 & 0.000 & 0.000 & 0.085\end{array}$

(+3)

$\begin{array}{lll}51 & 0.050 & 0.064\end{array}$

0.064

0.017

0.038

0.04

$\begin{array}{llll}36 & 0.035 & 0.039 & 0.061\end{array}$

0.067

0.000

0.000

0.036

0.023

PHLD_HUMAN

$\begin{array}{llllllll}92 & 0.018 & 0.031 & 0.024 & 0.021 & 0.055 & 0.036 & 0.031\end{array}$

0.014

F13A_HUMAN

$83 \quad 0.046$

ATRN_HUMAN

$159 \quad 0.016$

$\begin{array}{lll}0.046 & 0.000 \quad 0.037\end{array}$

Q65ZC9_HUMAN

$26 \quad 0.14$

0.041

0.02

0.022

0.033

0.030

QgUL70_HUMAN

$0.146 \quad 0.000$

Q9UL70_HUMAN

$\begin{array}{llllllll}12 & 0.000 & 0.000 & 0.000 & 0.000 & 0.000 & 0.137 & 0.023\end{array}$

0.022

$\begin{array}{lllllll}20 & 0.000 & 0.000 & 0.132 & 0.000 & 0.000 & 0.000\end{array}$

B4DF70_HUMAN (+2)

46

$\begin{array}{llllllllll}\text { A0A024R6N9_HUMAN (+1) } & 46 & 0.000 & 0.000 & 0.019 & 0.032 & 0.020 & 0.048 & 0.020 & 0.018\end{array}$

$\mathrm{GN}=\mathrm{ECM} 1 \mathrm{PE}=1 \mathrm{SV}=2$

Complement $\mathrm{C} 1 \mathrm{r}$ subcomponent-like protein

198 OS=Homo sapiens $\mathrm{GN}=\mathrm{C} 1 \mathrm{RL} P E=1 \mathrm{SV}=2$

V2-19 protein (Fragment) OS=Homo sapiens

$199 \mathrm{GN}=\mathrm{V} 2-19 \mathrm{PE}=4 \mathrm{SV}=1$

Myosin, heavy polypeptide 9, non-muscle,

isoform CRA_a OS=Homo sapiens GN=MYH9

$200 \quad P E=4 S V=1$

ECM1_HUMAN

C1RL_HUMAN

$\begin{array}{llll}61 & 0.014 & 0.000 & 0.022\end{array}$

0.040

0.000

0.000

0.036

0.019

$\begin{array}{llll}53 & 0.024 & 0.000 & 0.033\end{array}$

0.027

$0.026 \quad 0.000$

$0.000 \quad 0.018$

Q5NV91_HUMAN

$10 \quad 0.000$

$0.000 \quad 0.000 \quad 0.000$

0.000

0.000

A0A024R1N1_HUMAN (+1)

$\begin{array}{lcccccccrr}\text { A0A024R1N1_HUMAN (+1) } & 227 & 0.035 & 0.008 & 0.004 & 0.062 & 0.000 & 0.000 & 0.018 & 0.025 \\ \text { B4DUV1_HUMAN } & 70 & 0.030 & 0.040 & 0.019 & 0.000 & 0.020 & 0.000 & 0.018 & 0.016 \\ \text { HV308_HUMAN } & 13 & 0.000 & 0.108 & 0.000 & 0.000 & 0.000 & 0.000 & 0.018 & 0.044 \\ \text { FLNA_HUMAN } & 281 & 0.032 & 0.013 & 0.022 & 0.040 & 0.000 & 0.000 & 0.018 & 0.016 \\ \text { F2RM37_HUMAN (+1) } & 52 & 0.024 & 0.000 & 0.000 & 0.000 & 0.027 & 0.053 & 0.017 \\ \text { A0A024RAG6_HUMAN (+2) } & 26 & 0.049 & 0.054 & 0.000 & 0.000 & 0.000 & 0.000 & 0.021 \\ \text { FA11_HUMAN } & 70 & 0.012 & 0.013 & 0.025 & 0.021 & 0.000 & 0.031 & 0.017 \\ \text { APOM_HUMAN } & 21 & 0.101 & 0.000 & 0.000 & 0.000 & 0.000 & 0.000 & 0.017 \\ \end{array}$

0.035

$\begin{array}{lcccccccrr}\text { A0A024R1N1_HUMAN (+1) } & 227 & 0.035 & 0.008 & 0.004 & 0.062 & 0.000 & 0.000 & 0.018 & 0.025 \\ \text { B4DUV1_HUMAN } & 70 & 0.030 & 0.040 & 0.019 & 0.000 & 0.020 & 0.000 & 0.018 & 0.016 \\ \text { HV308_HUMAN } & 13 & 0.000 & 0.108 & 0.000 & 0.000 & 0.000 & 0.000 & 0.018 & 0.044 \\ \text { FLNA_HUMAN } & 281 & 0.032 & 0.013 & 0.022 & 0.040 & 0.000 & 0.000 & 0.018 & 0.016 \\ \text { F2RM37_HUMAN (+1) } & 52 & 0.024 & 0.000 & 0.000 & 0.000 & 0.027 & 0.053 & 0.017 \\ \text { A0A024RAG6_HUMAN (+2) } & 26 & 0.049 & 0.054 & 0.000 & 0.000 & 0.000 & 0.000 & 0.021 \\ \text { FA11_HUMAN } & 70 & 0.012 & 0.013 & 0.025 & 0.021 & 0.000 & 0.031 & 0.017 \\ \text { APOM_HUMAN } & 21 & 0.101 & 0.000 & 0.000 & 0.000 & 0.000 & 0.000 & 0.017 \\ \end{array}$

$\begin{array}{lcccccccrr}\text { A0A024R1N1_HUMAN (+1) } & 227 & 0.035 & 0.008 & 0.004 & 0.062 & 0.000 & 0.000 & 0.018 & 0.025 \\ \text { B4DUV1_HUMAN } & 70 & 0.030 & 0.040 & 0.019 & 0.000 & 0.020 & 0.000 & 0.018 & 0.016 \\ \text { HV308_HUMAN } & 13 & 0.000 & 0.108 & 0.000 & 0.000 & 0.000 & 0.000 & 0.018 & 0.044 \\ \text { FLNA_HUMAN } & 281 & 0.032 & 0.013 & 0.022 & 0.040 & 0.000 & 0.000 & 0.018 & 0.016 \\ \text { F2RM37_HUMAN (+1) } & 52 & 0.024 & 0.000 & 0.000 & 0.000 & 0.027 & 0.053 & 0.017 \\ \text { A0A024RAG6_HUMAN (+2) } & 26 & 0.049 & 0.054 & 0.000 & 0.000 & 0.000 & 0.000 & 0.021 \\ \text { FA11_HUMAN } & 70 & 0.012 & 0.013 & 0.025 & 0.021 & 0.000 & 0.031 & 0.017 \\ \text { APOM_HUMAN } & 21 & 0.101 & 0.000 & 0.000 & 0.000 & 0.000 & 0.000 & 0.017 \\ \end{array}$

$\begin{array}{lcccccccrr}\text { A0A024R1N1_HUMAN (+1) } & 227 & 0.035 & 0.008 & 0.004 & 0.062 & 0.000 & 0.000 & 0.018 & 0.025 \\ \text { B4DUV1_HUMAN } & 70 & 0.030 & 0.040 & 0.019 & 0.000 & 0.020 & 0.000 & 0.018 & 0.016 \\ \text { HV308_HUMAN } & 13 & 0.000 & 0.108 & 0.000 & 0.000 & 0.000 & 0.000 & 0.018 & 0.044 \\ \text { FLNA_HUMAN } & 281 & 0.032 & 0.013 & 0.022 & 0.040 & 0.000 & 0.000 & 0.018 & 0.016 \\ \text { F2RM37_HUMAN (+1) } & 52 & 0.024 & 0.000 & 0.000 & 0.000 & 0.027 & 0.053 & 0.017 \\ \text { A0A024RAG6_HUMAN (+2) } & 26 & 0.049 & 0.054 & 0.000 & 0.000 & 0.000 & 0.000 & 0.021 \\ \text { FA11_HUMAN } & 70 & 0.012 & 0.013 & 0.025 & 0.021 & 0.000 & 0.031 & 0.017 \\ \text { APOM_HUMAN } & 21 & 0.101 & 0.000 & 0.000 & 0.000 & 0.000 & 0.000 & 0.017 \\ \end{array}$

Fibulin-1 OS=Homo sapiens $\mathrm{PE}=2 \mathrm{SV}=1$

Ig heavy chain $V$-III region GA OS=Homo

202 sapiens $P E=1 \mathrm{SV}=1$

Filamin-A OS=Homo sapiens GN=FLNA PE=1

203 SV=4

$\mathrm{SV}=4$

$\mathrm{GN}=\mathrm{F} 9$ p22 $\mathrm{PE}=2 \mathrm{SV}=1$

Complement component 1, q subcomponent, $A$

chain, isoform CRA_a OS=Homo sapiens

$205 \mathrm{GN}=\mathrm{C} 1 \mathrm{QA} \mathrm{PE}=4 \mathrm{SV}=1$

Coagulation factor $\mathrm{XI} O \mathrm{OS}=\mathrm{Homo}$ sapiens

$206 \mathrm{GN}=\mathrm{F} 11 \mathrm{PE}=1 \mathrm{SV}=1$

Apolipoprotein M OS=Homo sapiens GN=APOM

$P E=1 \mathrm{SV}=2$

61 
Ig heavy chain variable region (Fragment)

208 OS=Homo sapiens $\mathrm{PE}=2 \mathrm{SV}=1$

Hepatocyte growth factor activator OS=Homo
sapiens $G N=H G F A C P E=1 S V=1$

210 HCG1782423 (Fragment) OS=Homo sapiens

$G N=I G L V 2-18 P E=4 S V=1$

Fibulin-1 OS=Homo sapiens GN=FBLN1 PE=1

211 SV=4

Complement factor $\mathrm{H}$-related protein 2

212 OS=Homo sapiens $G N=C F H R 2 P E=1 S V=1$ Fetuin-B OS=Homo sapiens $\mathrm{GN}=\mathrm{FETUB} P E=1$

$213 \quad S V=2$

214 AOA0F7TC28_HUMAN

Properdin OS=Homo sapiens $\mathrm{GN}=\mathrm{CFP} P E=1$

215 SV=2

Ig heavy chain $\mathrm{V}$-III region CAM OS=Homo

216 sapiens $P E=1 S V=1$

Ig kappa chain $V$-I region DEE OS=Homo

217 sapiens $P E=1 \mathrm{SV}=1$

Sex hormone-binding globulin OS=Homo

218 sapiens $\mathrm{GN}=\mathrm{SHBG} \mathrm{PE}=4 \mathrm{SV}=1$

Alpha-synuclein OS=Homo sapiens $\mathrm{GN}=\mathrm{SNCA}$

$219 \mathrm{PE}=1 \mathrm{SV}=1$

Sulfhydryl oxidase $1 \mathrm{OS}=$ Homo sapiens

$220 \mathrm{GN}=\mathrm{QSOX} 1 \mathrm{PE}=1 \mathrm{SV}=3$

C-reactive protein OS=Homo sapiens $\mathrm{GN}=\mathrm{CRP}$

$221 \quad P E=1 S V=1$

Peptidase inhibitor 16 OS=Homo sapiens

$222 \mathrm{GN}=\mathrm{PI} 16 \mathrm{PE}=1 \mathrm{SV}=1$

Fermitin family homolog 3 OS=Homo sapiens

$223 \mathrm{GN}=\mathrm{FERMT} 3 \mathrm{PE}=1 \mathrm{SV}=1$

IBM-A3 heavy chain variable region (Fragment)

224 OS=Homo sapiens $P E=2 \mathrm{SV}=1$

Catalase OS=Homo sapiens $\mathrm{GN}=\mathrm{CAT} P E=1$

$225 \quad S V=3$

Ficolin-2 OS=Homo sapiens $\mathrm{GN}=\mathrm{FCN} 2 \mathrm{PE}=1$

$226 \quad$ SV $=2$

Carbonic anhydrase 2 OS=Homo sapiens

$227 \mathrm{GN}=\mathrm{CA} 2 \mathrm{PE}=1 \mathrm{SV}=2$

Cartilage acidic protein $1 \mathrm{OS}=\mathrm{Homo}$ sapiens

$228 \mathrm{GN}=\mathrm{CRTAC} 1 \mathrm{PE}=1 \mathrm{SV}=2$

Vinculin, isoform CRA_c OS=Homo sapiens

$\mathrm{GN}=\mathrm{VCL} P E=4 \mathrm{SV}=1$

cDNA FLJ51023, highly similar to Vitamin K-

dependent protein $C(E C$ 3.4.21.69) OS=Homo

230 sapiens $\mathrm{PE}=2 \mathrm{SV}=1$

von Willebrand factor OS=Homo sapiens

$231 \mathrm{GN}=V W F \mathrm{PE}=1 \mathrm{SV}=4$

cDNA FLJ78437, highly similar to Homo sapiens

cartilage oligomeric matrix protein (COMP),

232 mRNA OS=Homo sapiens $\mathrm{PE}=2 \mathrm{SV}=1$

Conserved oligomeric Golgi complex subunit 3

233 OS=Homo sapiens GN=COG3 PE=1 SV=3

Macrophage stimulating 1 (Hepatocyte growth

factor-like) variant (Fragment) OS=Homo

234

sapiens $P E=2$ SV $=1$

$\begin{array}{llllllllll}\text { A0A068LRW6_HUMAN (+2) } & 14 & 0.000 & 0.000 & 0.000 & 0.000 & 0.099 & 0.000 & 0.017 & 0.041\end{array}$

$\begin{array}{llllllllll}\text { HGFA_HUMAN } & 71 & 0.024 & 0.020 & 0.019 & 0.014 & 0.000 & 0.023 & 0.017 & 0.009\end{array}$

$\begin{array}{lllllllllll}\text { A0A075B6J9_HUMAN (+1) } & 13 & 0.097 & 0.000 & 0.000 & 0.000 & 0.000 & 0.000 & 0.016 & 0.040\end{array}$

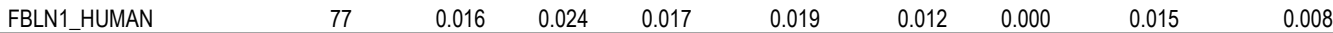

$\begin{array}{llllllllll}\text { FHR2_HUMAN (+1) } & 31 & 0.000 & 0.000 & 0.000 & 0.000 & 0.000 & 0.089 & 0.015 & 0.036\end{array}$

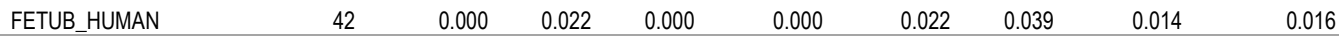

$\begin{array}{llllllllll}\text { A0A0F7TC28_HUMAN } & 11 & 0.000 & 0.000 & 0.080 & 0.000 & 0.000 & 0.000 & 0.013 & 0.033\end{array}$

$\begin{array}{llllllllll}\text { PROP_HUMAN (+1) } & 51 & 0.000 & 0.018 & 0.017 & 0.000 & 0.000 & 0.043 & 0.013 & 0.017\end{array}$

$\begin{array}{llllllllll}\text { HV307_HUMAN } & 14 & 0.000 & 0.000 & 0.000 & 0.000 & 0.000 & 0.078 & 0.013 & 0.032\end{array}$

$\begin{array}{llllllllll}\text { KV105_HUMAN } & 12 & 0.000 & 0.000 & 0.000 & 0.000 & 0.077 & 0.000 & 0.013 & 0.032\end{array}$

$\begin{array}{llllllllll}\text { I3L145_HUMAN (+1) } & 37 & 0.023 & 0.025 & 0.000 & 0.026 & 0.000 & 0.000 & 0.012 & 0.014\end{array}$

$\begin{array}{llllllllll}\text { E7EPV7_HUMAN (+3) } & 12 & 0.000 & 0.000 & 0.073 & 0.000 & 0.000 & 0.000 & 0.012 & 0.030\end{array}$

$\begin{array}{llllllllll}\text { QSOX1_HUMAN } & 83 & 0.010 & 0.017 & 0.011 & 0.017 & 0.017 & 0.000 & 0.012 & 0.007\end{array}$

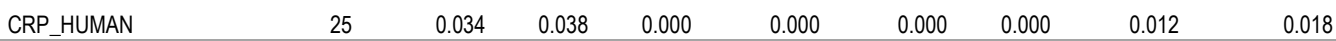

$\begin{array}{llllllllll}\text { PI16_HUMAN } & 49 & 0.017 & 0.000 & 0.000 & 0.020 & 0.000 & 0.034 & 0.012 & 0.014\end{array}$

$\begin{array}{llllllllll}\text { URP2_HUMAN } & 76 & 0.044 & 0.012 & 0.012 & 0.000 & 0.000 & 0.000 & 0.011 & 0.017\end{array}$

$\begin{array}{llllllllll}\text { AOAOX9UWM4_HUMAN } & 14 & 0.000 & 0.000 & 0.000 & 0.000 & 0.066 & 0.000 & 0.011 & 0.027\end{array}$

$\begin{array}{lllllllll}\text { CATA HUMAN } & 60 & 0.000 & 0.000 & 0.066 & 0.000 & 0.000 & 0.000 & 0.011\end{array}$

$\begin{array}{llllllllll}\text { FCN2_HUMAN } & 34 & 0.000 & 0.000 & 0.000 & 0.028 & 0.000 & 0.032 & 0.010 & 0.016\end{array}$

$\begin{array}{llllllllll}\text { CAH2_HUMAN (+2) } & 29 & 0.000 & 0.000 & 0.061 & 0.000 & 0.000 & 0.000 & 0.010 & 0.025\end{array}$

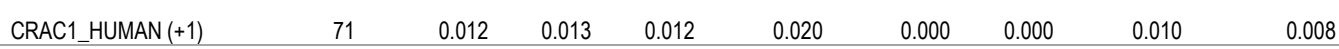

$\begin{array}{llllllllll}\text { A0A024QZN4_HUMAN (+2) } & 117 & 0.022 & 0.000 & 0.011 & 0.025 & 0.000 & 0.000 & 0.010 & 0.011\end{array}$

$\begin{array}{llllllllll}\text { B4DPC8_HUMAN (+4) } & 31 & 0.027 & 0.000 & 0.000 & 0.000 & 0.030 & 0.000 & 0.010 & 0.015\end{array}$

$\begin{array}{llllllllll}\text { VWF_HUMAN } & 309 & 0.019 & 0.005 & 0.031 & 0.000 & 0.000 & 0.000 & 0.009 & 0.013\end{array}$

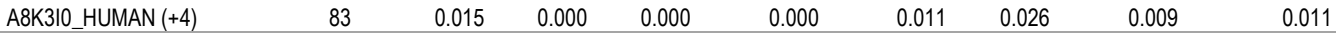

$\begin{array}{lllllllllll}\text { COG3_HUMAN } & 94 & 0.000 & 0.000 & 0.037 & 0.015 & 0.000 & 0.000 & 0.009 & 0.015\end{array}$

235 Zyxin OS=Homo sapiens GN=ZYX PE=1 SV=1 Proteoglycan 4, isoform CRA_a OS=Homo

236 sapiens $\mathrm{GN}=\mathrm{PRG} 4 \mathrm{PE}=4 \mathrm{SV}=1$

Butyrylcholinesterase, isoform CRA $b$

237 OS=Homo sapiens $\mathrm{GN}=\mathrm{BCHE} \mathrm{PE}=3 \mathrm{SV}=1$

cDNA FLJ78503, highly similar to Homo sapiens

keratin 13 (KRT13), transcript variant 1, mRNA

238 OS=Homo sapiens PE=2 SV=1

$\mathrm{GN}=\mathrm{KRT} 5 \mathrm{PE}=1 \mathrm{SV}=3$

Extracellular link domain containing $1 \mathrm{OS}=\mathrm{Homo}$

240 sapiens $\mathrm{GN}=X L K D 1 \mathrm{PE}=2 \mathrm{SV}=1$

Stomatin, isoform CRA a OS=Homo sapiens

$241 \mathrm{GN}=\mathrm{STOM} P E=4 \mathrm{SV}=1$

$\mathrm{GN}=\mathrm{STOM} P E=4 \mathrm{SV}=1$

242 GN=PKM PE=1 SV=4

Mannosyl-oligosaccharide 1,2-alpha-

mannosidase IA OS=Homo sapiens

$243 \mathrm{GN}=\mathrm{MAN} 1 \mathrm{~A} 1 \mathrm{PE}=1 \mathrm{SV}=3$

\begin{tabular}{|c|c|c|c|c|c|c|c|c|c|}
\hline Q53GN8_HUMAN & 80 & 0.021 & 0.018 & 0.000 & 0.000 & 0.000 & 0.014 & 0.009 & 0.010 \\
\hline ZYX_HUMAN & 61 & 0.028 & 0.000 & 0.000 & 0.016 & 0.000 & 0.000 & 0.007 & 0.012 \\
\hline A0A024R930_HUMAN (+2) & 151 & 0.000 & 0.012 & 0.006 & 0.000 & 0.012 & 0.011 & 0.007 & 0.006 \\
\hline D3DNN4_HUMAN & 73 & 0.000 & 0.000 & 0.012 & 0.013 & 0.013 & 0.000 & 0.006 & 0.007 \\
\hline A8K2H9_HUMAN & 50 & 0.000 & 0.038 & 0.000 & 0.000 & 0.000 & 0.000 & 0.006 & 0.015 \\
\hline K2C5_HUMAN & 62 & 0.000 & 0.000 & 0.000 & 0.000 & 0.037 & 0.000 & 0.006 & 0.015 \\
\hline B2R672_HUMAN (+1) & 35 & 0.000 & 0.000 & 0.000 & 0.000 & 0.000 & 0.031 & 0.005 & 0.013 \\
\hline A0A024R882_HUMAN (+2) & 32 & 0.000 & 0.000 & 0.000 & 0.030 & 0.000 & 0.000 & 0.005 & 0.012 \\
\hline KPYM_HUMAN (+1) & 58 & 0.029 & 0.000 & 0.000 & 0.000 & 0.000 & 0.000 & 0.005 & 0.012 \\
\hline
\end{tabular}




$\begin{array}{llllllllll} & 0.000 & 0.000 & 0.013 & 0.000 & 0.000 & 0.000 & 0.002 & & \end{array}$

$\begin{array}{llllllllll}\text { V9HWG7_HUMAN (+1) } & 66 & 0.013 & 0.000 & 0.000 & 0.000 & 0.000 & 0.000 & 0.002 & 0.005\end{array}$

$\begin{array}{llllllllll}\text { DOPO_HUMAN } & 69 & 0.000 & 0.000 & 0.013 & 0.000 & 0.000 & 0.000 & 0.002 & 0.005\end{array}$

$253 \quad P E=1 S V=2$

lgGFc-binding protein OS=Homo sapiens

$\mathrm{GN}=\mathrm{FCGBP} P E=1 \mathrm{SV}=3$

Mannan-binding lectin serine protease 1

Spectrin sapiens $G N=M A S P 1 P E=1 S V=3$

DTS

ITB3 HUMAN (+1)

87

FCGBP_HUMAN

8.0 .000

$\begin{array}{llllllll}0.000 & 0.000 & 0.000 & 0.011 & 0.000 & 0.000 & 0.002 & 0.005\end{array}$

sapiens $\mathrm{GN}=\mathrm{SPTA} 1 \mathrm{PE}=1 \mathrm{SV}=1$

257

Ankyrin-1 OS=Homo sapiens GN=ANK1 PE=1

SV $=3$

MASP1_HUMAN

$572 \quad 0.001$

$0.001-0.003$

$79 \quad 0.011$

$0.001 \quad 0.003 \quad 0.002$

$0.002-0.002$

A0A087WZE4_HUMAN (+1) 281

Spectrin, beta, erythrocytic (Includes

spherocytosis, clinical type I) variant (Fragment)

258

$\mathrm{OS}=$ Homo sapiens $\mathrm{PE}=2 \mathrm{SV}=1$

ANK1 HUMAN

$\begin{array}{llll}281 & 0.000 & 0.000 & 0.005\end{array}$

0.000

0.002

$0.000 \quad 0.003$

$0.003 \quad 0.002$

$0.002 \quad 0.001$

$\begin{array}{llll}206 & 0.000 & 0.000 & 0.004\end{array}$

0.000

$\begin{array}{lll}0.000 & 0.000 \quad 0.002\end{array}$
0.004

268

$\begin{array}{lll}0.000 & 0.000 \quad 0.003\end{array}$

\begin{tabular}{lllll|}
0.000 & 0.000 & 0.000 & 0.001 & 0.002 \\
0.000 & 0.000 & 0.000 & 0.001 & 0.002 \\
\hline 0.000 & 0.000 & 0.000 & 0.001 & 0.001 \\
\hline
\end{tabular}


Table S5: Characterization of the in vivo protein corona. Full list of common proteins identified in the liposomal protein corona in all 6 ovarian carcinoma patients.

\begin{tabular}{|c|c|c|c|c|}
\hline$\#$ & Identified protein & Accession Number & $\begin{array}{l}\text { Average } \\
\text { RPA } \%\end{array}$ & STV \\
\hline 1 & Full-length cDNA clone CS0DD006YL02 of Neuroblastoma of Homo sapiens (human) OS=Homo sapiens PE=2 SV=1 & Q86TT1_HUMAN & 7.98 & 2.44 \\
\hline 2 & Ig mu chain $\mathrm{C}$ region OS=Homo sapiens $\mathrm{GN}=\mathrm{IGHM} \mathrm{PE}=1 \mathrm{SV}=1$ & A0A087X2C0_HUMAN & 5.70 & 1.78 \\
\hline 3 & Alpha-2-macroglobulin OS=Homo sapiens GN=A2M PE=1 SV=3 & A2MG_HUMAN & 3.47 & 1.01 \\
\hline 4 & Fibrinogen beta chain OS=Homo sapiens GN=FGB PE=1 SV=2 & FIBB_HUMAN (+1) & 3.46 & 1.20 \\
\hline 5 & Fibrinogen alpha chain OS=Homo sapiens GN=FGA PE=1 SV=2 & FIBA_HUMAN & 2.99 & 0.67 \\
\hline 6 & Apolipoprotein B (Including $\mathrm{Ag}(\mathrm{X})$ antigen) OS=Homo sapiens GN=APOB PE=4 SV=1 & COJYY2_HUMAN & 2.64 & 1.43 \\
\hline 7 & Serum albumin OS=Homo sapiens GN=ALB PE=1 SV=2 & ALBU_HUMAN & 2.48 & 2.08 \\
\hline 8 & Protein IGHV3-72 OS=Homo sapiens GN=IGHV3-72 PE=4 SV=1 & A0A087WW89_HUMAN & 1.91 & 0.57 \\
\hline 9 & C4b-binding protein alpha chain OS=Homo sapiens $\mathrm{GN}=\mathrm{C} 4 \mathrm{BPA} P \mathrm{PE}=1 \mathrm{SV}=2$ & C4BPA_HUMAN & 1.74 & 0.59 \\
\hline 10 & Fibrinogen gamma chain OS=Homo sapiens $\mathrm{GN}=\mathrm{FGG} P E=1 \mathrm{SV}=3$ & FIBG_HUMAN & 1.60 & 0.54 \\
\hline 11 & Haptoglobin (Fragment) OS=Homo sapiens GN=HP PE=1 SV=1 & H3BS21_HUMAN & 1.36 & 0.35 \\
\hline 12 & Haptoglobin OS=Homo sapiens GN=HP PE=1 SV=4 & HOY300_HUMAN & 1.35 & 0.44 \\
\hline 13 & IGH@ protein OS=Homo sapiens GN=IGH@ PE=1 SV=1 & Q6GMX6_HUMAN & 1.14 & 0.65 \\
\hline 14 & Ig gamma-1 chain $\mathrm{C}$ region $\mathrm{OS}=$ Homo sapiens $\mathrm{GN}=\mid \mathrm{GHG} 1 \mathrm{PE}=1 \mathrm{SV}=1$ & A0A087WV47_HUMAN (+1) & 1.13 & 0.60 \\
\hline 15 & cDNA FLJ78387 OS=Homo sapiens PE=1 SV=1 & A8K008_HUMAN & 1.12 & 0.60 \\
\hline 16 & Putative uncharacterized protein DKFZp686P15220 OS=Homo sapiens GN=DKFZp686P15220 PE=1 SV=1 & Q6N089_HUMAN & 1.06 & 0.61 \\
\hline 17 & IgG $\mathrm{H}$ chain $\mathrm{OS}=\mathrm{Homo}$ sapiens $\mathrm{PE}=1 \mathrm{SV}=1$ & S6B291_HUMAN & 1.05 & 0.65 \\
\hline 18 & Actin, cytoplasmic 1 OS=Homo sapiens GN=ACTB PE=1 SV=1 & ACTB_HUMAN (+2) & 1.04 & 0.60 \\
\hline 19 & Ig gamma-1 chain $\mathrm{C}$ region OS=Homo sapiens $\mathrm{GN}=\mid \mathrm{GHG} 1 \mathrm{PE}=1 \mathrm{SV}=1$ & A0A087WYC5_HUMAN & 0.96 & 0.63 \\
\hline 20 & GCT-A10 heavy chain variable region (Fragment) OS=Homo sapiens PE=2 SV=1 & A0A120HG46_HUMAN & 0.96 & 0.51 \\
\hline 21 & $\begin{array}{l}\text { cDNA FLJ14473 fis, clone MAMMA1001080, highly similar to Homo sapiens SNC73 protein (SNC73) mRNA OS=Homo sapiens } \\
\text { PE=2 SV=1 }\end{array}$ & Q96K68_HUMAN & 0.94 & 0.30 \\
\hline 22 & Haptoglobin-related protein OS=Homo sapiens GN=HPR PE=1 SV=2 & HPTR_HUMAN & 0.93 & 0.22 \\
\hline 23 & Putative uncharacterized protein DKFZp686G11190 OS=Homo sapiens GN=DKFZp686G11190 PE=1 SV=1 & Q6MZQ6_HUMAN & 0.92 & 0.61 \\
\hline 24 & Myosin-reactive immunoglobulin heavy chain variable region (Fragment) OS=Homo sapiens PE=2 SV=1 & Q9UL90_HUMAN & 0.89 & 0.22 \\
\hline 25 & Putative uncharacterized protein DKFZp686C15213 OS=Homo sapiens GN=DKFZp686C15213 PE=1 SV=1 & Q6MZU6_HUMAN & 0.87 & 0.64 \\
\hline 26 & Ig gamma-3 chain C region OS=Homo sapiens GN=IGHG3 PE=4 SV=1 & A0A087WXL8_HUMAN & 0.86 & 0.65 \\
\hline 27 & Apolipoprotein E OS=Homo sapiens GN=APOE PE=1 SV=1 & APOE_HUMAN (+1) & 0.83 & 0.48 \\
\hline 28 & IGL@ protein OS=Homo sapiens GN=IGL@ PE=1 SV=1 & Q8N355_HUMAN & 0.80 & 0.18 \\
\hline 29 & IGK@ protein OS=Homo sapiens GN=IGK@ PE=1 SV=1 & Q6PIL8_HUMAN & 0.77 & 0.21 \\
\hline 30 & Complement C3 OS=Homo sapiens GN=C3 PE=1 SV=2 & CO3_HUMAN (+1) & 0.77 & 0.43 \\
\hline 31 & Hemoglobin, beta OS=Homo sapiens GN=HBB PE=3 SV=1 & D9YZU5_HUMAN (+1) & 0.64 & 0.44 \\
\hline 32 & MS-D4 heavy chain variable region (Fragment) OS=Homo sapiens PE=2 SV=1 & A0A0X9UWK7_HUMAN & 0.63 & 0.13 \\
\hline 33 & Anti-FactorVIII scFv (Fragment) OS=Homo sapiens PE=2 SV=1 & A2KBC6_HUMAN & 0.61 & 0.22 \\
\hline 34 & Alpha-2 globin chain OS=Homo sapiens GN=HBA2 PE=3 SV=1 & D1MGQ2_HUMAN (+2) & 0.59 & 0.31 \\
\hline 35 & Protein IGHV3-74 (Fragment) OS=Homo sapiens GN=IGHV3-74 PE=1 SV=1 & A0A0B4J1X5_HUMAN & 0.58 & 0.21 \\
\hline 36 & Vitamin K-dependent protein S OS=Homo sapiens GN=PROS1 PE=1 SV=1 & PROS_HUMAN (+2) & 0.57 & 0.17 \\
\hline 37 & Transferrin variant (Fragment) OS=Homo sapiens $\mathrm{PE}=2 \mathrm{SV}=1$ & Q53H26_HUMAN & 0.51 & 0.39 \\
\hline
\end{tabular}




\begin{tabular}{|c|c|c|c|c|}
\hline 38 & Galectin-3-binding protein OS=Homo sapiens $\mathrm{GN}=\mathrm{LGALS3BP} P E=1 \mathrm{SV}=1$ & LG3BP_HUMAN (+1) & 0.50 & 0.32 \\
\hline 39 & Apolipoprotein C-III OS=Homo sapiens GN=APOC3 PE=2 SV=1 & A3KPE2_HUMAN (+2) & 0.50 & 0.40 \\
\hline 40 & 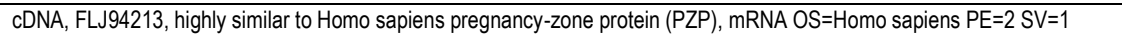 & B2R950_HUMAN (+1) & 0.49 & 0.08 \\
\hline 41 & Thrombospondin-1 OS=Homo sapiens $\mathrm{GN}=\mathrm{THBS1} \mathrm{PE}=1 \mathrm{SV}=2$ & TSP1_HUMAN & 0.48 & 0.26 \\
\hline 42 & Protein IGHV5-51 (Fragment) OS=Homo sapiens GN=IGHV5-51 PE=1 SV=1 & A0A0C4DH38_HUMAN & 0.47 & 0.10 \\
\hline 43 & Vinculin, isoform CRA_c OS=Homo sapiens GN=VCL PE=4 SV=1 & A0A024QZN4_HUMAN (+2) & 0.46 & 0.28 \\
\hline 44 & Fibronectin 1, isoform CRA_n OS=Homo sapiens GN=FN1 PE=4 SV=1 & A0A024R462_HUMAN & 0.46 & 0.11 \\
\hline 45 & Keratin $1 \mathrm{OS}=$ Homo sapiens $\mathrm{GN}=\mathrm{KRT} 1 \mathrm{PE}=3 \mathrm{SV}=1$ & H6VRF8_HUMAN (+3) & 0.44 & 0.08 \\
\hline 46 & Filamin-A OS=Homo sapiens GN=FLNA PE=1 SV=4 & FLNA_HUMAN & 0.42 & 0.27 \\
\hline 47 & Alpha-1-antitrypsin OS=Homo sapiens GN=SERPINA1 PE=1 SV=3 & A1AT_HUMAN (+1) & 0.41 & 0.24 \\
\hline 48 & Ig heavy chain V-III region BUT OS=Homo sapiens $P E=1 \mathrm{SV}=1$ & HV306_HUMAN & 0.41 & 0.20 \\
\hline 49 & Talin-1 OS=Homo sapiens GN=TLN1 PE=1 SV=3 & TLN1_HUMAN & 0.41 & 0.24 \\
\hline 50 & Actinin, alpha 1, isoform CRA_a OS=Homo sapiens GN=ACTN1 PE=4 SV=1 & A0A024R694_HUMAN (+1) & 0.41 & 0.23 \\
\hline 51 & Apolipoprotein A-l, isoform CRA_a OS=Homo sapiens GN=APOA1 PE=4 SV=1 & A0A024R3E3_HUMAN (+1) & 0.40 & 0.11 \\
\hline 52 & von Willebrand factor OS=Homo sapiens GN=VWF PE=1 SV=4 & VWF_HUMAN & 0.39 & 0.25 \\
\hline 53 & Coagulation factor $\mathrm{XIII} A$ chain OS=Homo sapiens $\mathrm{GN}=\mathrm{F} 13 \mathrm{~A} 1 \mathrm{PE}=1 \mathrm{SV}=4$ & F13A_HUMAN & 0.36 & 0.23 \\
\hline 54 & Complement factor H OS=Homo sapiens GN=CFH PE=1 SV=4 & CFAH_HUMAN & 0.33 & 0.12 \\
\hline 55 & Keratin, type I cytoskeletal 10 OS=Homo sapiens GN=KRT10 PE=1 SV=6 & K1C10_HUMAN & 0.30 & 0.10 \\
\hline 56 & Protein APOC4-APOC2 OS=Homo sapiens GN=APOC4-APOC2 PE=4 SV=1 & K7ER74_HUMAN & 0.30 & 0.16 \\
\hline 57 & Keratin, type I cytoskeletal 9 OS=Homo sapiens GN=KRT9 PE=1 SV=3 & K1C9_HUMAN & 0.28 & 0.09 \\
\hline 58 & Epididymis tissue protein Li 173 OS=Homo sapiens $\mathrm{PE}=2 \mathrm{SV}=1$ & E9KL26_HUMAN (+1) & 0.27 & 0.15 \\
\hline 59 & Myosin, heavy polypeptide 9, non-muscle, isoform CRA_a OS=Homo sapiens GN=MYH9 PE=4 SV=1 & A0A024R1N1_HUMAN (+1) & 0.26 & 0.22 \\
\hline 60 & Complement $\mathrm{C} 1 \mathrm{r}$ subcomponent $\mathrm{OS}=\mathrm{Homo}$ sapiens $\mathrm{GN}=\mathrm{C} 1 \mathrm{R} \mathrm{PE}=2 \mathrm{SV}=1$ & B4DPQ0_HUMAN & 0.26 & 0.08 \\
\hline 61 & Heat shock cognate $71 \mathrm{kDa}$ protein OS=Homo sapiens GN=HSPA8 PE=1 SV=1 & HSP7C_HUMAN (+1) & 0.24 & 0.16 \\
\hline 62 & Hemopexin OS=Homo sapiens GN=HPX PE=1 SV=2 & HEMO_HUMAN & 0.23 & 0.21 \\
\hline 63 & Epididymis secretory protein Li 52 OS=Homo sapiens $\mathrm{GN}=\mathrm{HEL}-\mathrm{S}-52 \mathrm{PE}=2 \mathrm{SV}=1$ & V9HWG7_HUMAN (+1) & 0.22 & 0.15 \\
\hline 64 & Keratin, type Il cytoskeletal 2 epidermal OS=Homo sapiens GN=KRT2 PE=1 SV=2 & K22E_HUMAN & 0.22 & 0.07 \\
\hline 65 & Fermitin family homolog 3 OS=Homo sapiens GN=FERMT3 PE=1 SV=1 & URP2_HUMAN & 0.21 & 0.14 \\
\hline 66 & Complement C4-B OS=Homo sapiens GN=C4B PE=1 SV=2 & CO4B_HUMAN & 0.21 & 0.09 \\
\hline 67 & Pyruvate kinase PKM OS=Homo sapiens GN=PKM PE=1 SV=4 & KPYM_HUMAN (+1) & 0.21 & 0.12 \\
\hline 68 & Gelsolin OS=Homo sapiens GN=GSN PE=1 SV=1 & GELS_HUMAN & 0.20 & 0.10 \\
\hline 69 & CD5 antigen-like OS=Homo sapiens $G N=C D 5 L P E=1 S V=1$ & CD5L_HUMAN & 0.19 & 0.08 \\
\hline 70 & Immunglobulin heavy chain variable region (Fragment) $\mathrm{OS}=$ Homo sapiens $\mathrm{PE}=4 \mathrm{SV}=1$ & Q0ZCH6_HUMAN & 0.18 & 0.05 \\
\hline 71 & Complement component 1 , $q$ subcomponent, $C$ chain, isoform CRA_a OS=Homo sapiens GN=C1QC PE=4 SV=1 & A0A024RAA7_HUMAN (+1) & 0.18 & 0.03 \\
\hline 72 & Tubulin alpha-4A chain OS=Homo sapiens GN=TUBA4A PE=1 SV=1 & TBA4A_HUMAN & 0.18 & 0.11 \\
\hline 73 & cDNA FLJ35730 fis, clone TESTI2003131, highly similar to ALPHA-1-ANTICHYMOTRYPSIN OS=Homo sapiens PE=2 SV=1 & B3KS79_HUMAN & 0.17 & 0.12 \\
\hline 74 & Immunoglobulin $\mathrm{J}$ chain OS=Homo sapiens GN=IGJ PE=1 SV=4 & IGJ_HUMAN & 0.16 & 0.07 \\
\hline 75 & Inter-alpha-trypsin inhibitor heavy chain H2 OS=Homo sapiens GN=ITIH2 PE=1 SV=1 & Q5T985_HUMAN & 0.16 & 0.05 \\
\hline 76 & $78 \mathrm{kDa}$ glucose-regulated protein OS=Homo sapiens $\mathrm{GN}=\mathrm{HSPA} 5 \mathrm{PE}=1 \mathrm{SV}=2$ & GRP78_HUMAN (+1) & 0.16 & 0.14 \\
\hline 77 & Moesin OS=Homo sapiens GN=MSN PE=1 SV=3 & MOES_HUMAN (+1) & 0.16 & 0.10 \\
\hline 78 & Apolipoprotein M OS=Homo sapiens GN=APOM PE=1 SV=2 & APOM_HUMAN & 0.15 & 0.08 \\
\hline 79 & Complement component 1 , $q$ subcomponent, $B$ chain, isoform CRA_a OS=Homo sapiens $G N=C 1 Q B P E=4 S V=1$ & A0A024RAB9_HUMAN (+3) & 0.15 & 0.06 \\
\hline 80 & Beta 5-tubulin OS=Homo sapiens GN=TUBB PE=2 SV=1 & Q5SU16_HUMAN (+1) & 0.15 & 0.14 \\
\hline
\end{tabular}




\section{Supporting Table 6}

Table S6: Most-abundant proteins (top-20) identified by LC-MS/MS in ex vivo protein coronas (formed after incubation of PEGylated liposomal doxorubicin with plasma samples) obtained from ovarian carcinoma patients $(n=6)$ and from healthy controls $(n=6)$. RPA\% values represent the average and standard deviation from the 6 biological replicates.

\begin{tabular}{|c|c|c|c|}
\hline \multicolumn{2}{|l|}{ Ex vivo corona (Ovarian Carcinoma Patients; $\mathrm{n}=6$ ) } & \multicolumn{2}{|l|}{ Ex vivo corona (Healthy Controls; $\mathrm{n}=6$ ) } \\
\hline Identified Protein & RPA\% & Identified Protein & RPA\% \\
\hline Full-length cDNA clone CSODD006YL02 of Neuroblas toma of Homo sapiens & $4.79 \pm 0.92$ & Apolipoprotein B (Including $\mathrm{Ag}(\mathrm{X})$ antigen) & $4.60 \pm 2.55$ \\
\hline Apolipoprotein B (Including $\mathrm{Ag}(\mathrm{X})$ antigen) & $3.49 \pm 1.27$ & Alpha-2-macroglobulin & $4.56 \pm 2.11$ \\
\hline Ig mu chain $\mathrm{C}$ region (A0A087X2C0) & $3.44 \pm 0.74$ & Ig mu chain $\mathrm{C}$ region $(\mathrm{A} 0 \mathrm{~A} 087 \mathrm{X} 2 \mathrm{C} 0)$ & $4.53 \pm 0.77$ \\
\hline Fibrinogen beta chain & $3.11 \pm 0.87$ & Fibrinogen beta chain & $3.69 \pm 0.60$ \\
\hline Haptoglobin & $3.10 \pm 1.22$ & Full-length cDNA clone CSODD006YL02 of Neuroblas toma of Homo sapiens & $3.43 \pm 3.80$ \\
\hline Haptoglobin (Fragment) (H3BS21) & $3.05 \pm 1.15$ & Ig mu chain C region (A0A087WYJ9) & $3.03 \pm 2.41$ \\
\hline Ig mu chain $\mathrm{C}$ region (A0A087WYJ9) & $2.65 \pm 1.45$ & Fibrinogen alpha chain & $2.80 \pm 1.07$ \\
\hline Beta-globin (D9YZU5) & $2.43 \pm 1.29$ & IGK@ protein (Q6PIL8) & $2.15 \pm 0.15$ \\
\hline Fibrinogen alpha chain & $2.42 \pm 0.86$ & cDNA FLJ51597, highly similar to C4b-binding protein alpha chain & $2.12 \pm 1.17$ \\
\hline Haptoglobin-related protein (HPTR) & $2.28 \pm 0.99$ & Fibrinogen gamma chain & $2.09 \pm 0.74$ \\
\hline Apolipoprotein E isoform 1 (Fragment) & $2.08 \pm 0.84$ & Lipoprotein B (Fragment) & $2.09 \pm 3.25$ \\
\hline Mutant hemoglobin alpha 2 globin chain (A0A0K2BMD8) & $2.07 \pm 1.47$ & Haptoglobin & $2.06 \pm 1.02$ \\
\hline Fibrinogen gamma chain & $1.98 \pm 0.54$ & Lambda-chain (AA -20 to 215) & $1.99 \pm 0.16$ \\
\hline Lipoprotein B (Fragment) & $1.94 \pm 4.74$ & Haptoglobin (Fragment) (H3BS21) & $1.99 \pm 1.31$ \\
\hline Protein IGHV3-72 & $1.92 \pm 0.74$ & Uncharacterized protein (Q8TCD0) & $1.69 \pm 0.84$ \\
\hline cDNA FLJ51597, highly similar to C4b-binding protein alpha chain & $1.78 \pm 0.53$ & Haptoglobin-related protein (HPTR) & $1.55 \pm 0.73$ \\
\hline Lambda-chain (AA -20 to 215) & $1.41 \pm 0.20$ & Apolipoprotein E isoform 1 (Fragment) & $1.28 \pm 0.46$ \\
\hline Serum albumin & $1.37 \pm 0.32$ & Ig gamma-1 chain $C$ region (A0A087WV47) & $1.18 \pm 0.40$ \\
\hline
\end{tabular}

